\title{
Volume Polynomials and Duality Algebras of Multi-Fans
}

\author{
Anton Ayzenberg1 (D . Mikiya Masuda ${ }^{2}$
}

Received: 17 October 2015 / Revised: 12 November 2015 / Accepted: 23 June 2016 /

Published online: 11 July 2016

(C) Institute for Mathematical Sciences (IMS), Stony Brook University, NY 2016

\begin{abstract}
We introduce a theory of volume polynomials and corresponding duality algebras of multi-fans. Any complete simplicial multi-fan $\Delta$ determines a volume polynomial $V_{\Delta}$ whose values are the volumes of multi-polytopes based on $\Delta$. This homogeneous polynomial is further used to construct a Poincare duality algebra $\mathcal{A}^{*}(\Delta)$. We study the structure and properties of $V_{\Delta}$ and $\mathcal{A}^{*}(\Delta)$ and give applications and connections to other subjects, such as Macaulay duality, Novik-Swartz theory of face rings of simplicial manifolds, generalizations of Minkowski's theorem on convex polytopes, cohomology of torus manifolds, computations of volumes, and linear relations on the powers of linear forms. In particular, we prove that the analogue of the $g$-theorem does not hold for multi-polytopes.
\end{abstract}

Keywords Multi-fan · Multi-polytope · Volume polynomial · Poincare duality algebra $\cdot$ Macaulay duality $\cdot$ Stanley-Reisner ring $\cdot$ Minkowski theorem · Minkowski relations · Torus manifold

Mathematics Subject Classification 52A39 - 52B11 - 05E45 - 52C35 - 05E40 . $13 \mathrm{H} 10 \cdot 52 \mathrm{~B} 05 \cdot 52 \mathrm{~B} 40 \cdot 52 \mathrm{~B} 70 \cdot 57 \mathrm{~N} 65 \cdot 55 \mathrm{~N} 91 \cdot 28 \mathrm{~A} 75 \cdot 51 \mathrm{M} 25 \cdot 13 \mathrm{~A} 02$

Anton Ayzenberg

ayzenberga@gmail.com; aizenbergaa@yandex.ru

1 Faculty of Mathematics, National Research University Higher School of Economics, Moscow, Russia

2 Department of Mathematics, Osaka City University, Osaka, Japan 


\section{Introduction}

There is a fundamental correspondence in algebraic geometry (Fulton 1993):

$$
\left\{\begin{array}{c}
\text { Toric varieties } \\
\text { of complex dimension } n
\end{array}\right\} \leadsto\left\{\text { Rational fans in } \mathbb{R}^{n}\right\} .
$$

One can read the information about toric variety from its fan. Complete toric varieties correspond to complete fans, non-singular varieties correspond to non-singular fans, and projective toric varieties correspond to normal fans of convex polytopes. Combinatorics of a fan and geometry of a toric variety are closely connected. In particular, the rays of a fan correspond to the divisors on toric variety and higher dimensional cones correspond to the intersections of divisors.

In the work Hattori and Masuda (2003) expanded this setting to topological category and generalized the above-mentioned correspondence in the following way:

$$
\left\{\begin{array}{c}
\text { Torus manifolds } \\
\text { of real dimension } 2 n
\end{array}\right\} \rightsquigarrow\left\{\text { Nonsingular multi-fans in } \mathbb{R}^{n}\right\},
$$

which will be explained in a minute.

Let $X$ be a smooth closed oriented $2 n$-manifold with an effective action of an $n$-dimensional compact torus $T$ and at least one fixed point. A closed, connected, codimension two submanifold of $X$ will be called characteristic if it is a connected component of the fixed point set of a certain circle subgroup $S$ of $T$, and if it contains at least one $T$-fixed point. The manifold $X$ together with a preferred orientation of each characteristic submanifold is called a torus manifold. Characteristic submanifolds are the analogues of divisors on a toric variety.

Note, that there is no one-to-one correspondence in (1.1): there may be different (in any sense) torus manifolds producing the same multi-fan. Nevertheless, multi-fans provide a convenient tool to study such manifolds.

A multi-fan is the central object of this paper. We recall the precise definition later. Informally, a multi-fan is a collection of cones in $V \cong \mathbb{R}^{n}$ with apex at the origin, coming with multiplicities and satisfying certain restrictions. Sometimes it is convenient to assume that there is a fixed lattice $N \subset V$, and the rays of $\Delta$ are rational with respect to $N$. The cones of a multi-fan may overlap nontrivially, which makes a multi-fan more general and flexible object than an ordinary fan, and provides many nontrivial examples.

A multi-polytope is defined as follows. Let $\Delta$ be a simplicial multi-fan in $V \cong \mathbb{R}^{n}$. For each ray $l_{i} \in \Delta$, we specify an affine hyperplane $H_{i} \subset V^{*}$ orthogonal to the linear span of $l_{i}$. A tuple $P=\left(\Delta, H_{1}, \ldots, H_{m}\right)$ is called a simple multi-polytope based on $\Delta$. The relation of the multi-polytope to the multi-fan on which it is based, is exactly the same as the relation of a polytope to its normal fan.

For any multi-polytope $P \subset V^{*}$ there is a function $\mathrm{DH}_{P}: V^{*} \backslash \bigcup H_{i} \rightarrow \mathbb{Z}$ (the notation stands for Duistermaat-Heckman, see Hattori and Masuda 2003). Informally, for a generic point $x \in V^{*}$ the value $\mathrm{DH}_{P}(x)$ indicates how many times the "boundary" 
of $P$ wraps around $x$. The precise definition is given in Sect. 3. For an ordinary simple convex polytope this function takes value 1 inside the polytope, and 0 outside.

A multi-fan $\Delta$ is called complete if it satisfies certain mild conditions (see Hattori and Masuda 2003 or Definition 2.5 below). For multi-polytopes based on complete simplicial multi-fans, the function $\mathrm{DH}_{P}$ is compactly supported. We can define the volume of a multi-polytope $P$ as an integral

$$
\operatorname{Vol}(P):=\int_{V^{*}} \mathrm{DH}_{P} d \mu
$$

(the measure $\mu$ is chosen such that the volume of a fundamental domain of the dual lattice $N^{*}$ is 1 ).

For a given simplicial multi-fan $\Delta$ consider the space Poly $(\Delta)$ of all multi-polytopes based on $\Delta$. Following Timorin (1999) we call it the space of analogous multipolytopes. To specify an affine hyperplane orthogonal to a line $\left\langle l_{i}\right\rangle \subset V$ one needs a single real number $c_{i}$, the normalized distance from $H_{i}$ to the origin taken with sign. This number is called the support parameter. Thus the space $\operatorname{Poly}(\Delta)$ is isomorphic to $\mathbb{R}^{m}$, where $m$ is the number of rays of $\Delta$. Support parameters $\left(c_{1}, \ldots, c_{m}\right)$ provide the canonical coordinates on $\operatorname{Poly}(\Delta)$.

If $\Delta$ is complete, the volume gives a function on the space of analogous polytopes: $\operatorname{Poly}(\Delta) \rightarrow \mathbb{R}, P \mapsto \operatorname{Vol}(P)$. Similarly to the case of actual convex polytopes, studied by Pukhlikov-Khovanskii (Khovanskii and Pukhlikov 1992a), this function is a homogeneous polynomial in the support parameters.

Theorem 1.1 (Hattori and Masuda 2003) Let $\Delta$ be a complete simplicial multi-fan in $\mathbb{R}^{n}$ with $m$ rays. There exists a homogeneous polynomial $V_{\Delta} \in \mathbb{R}\left[c_{1}, \ldots, c_{m}\right]$ of degree $n$ such that $V_{\Delta}\left(c_{1}, \ldots, c_{m}\right)=\operatorname{Vol}(P)$ for a multi-polytope $P \in \operatorname{Poly}(\Delta)$ with support parameters $\left(c_{1}, \ldots, c_{m}\right)$.

Following the approach introduced in Khovanskii and Pukhlikov (1992b) and developed in Timorin (1999), we proceed as follows. Consider the ring $\mathcal{D}$ of differential operators with constant coefficients, acting on $\mathbb{R}\left[c_{1}, \ldots, c_{m}\right]$. We have $\mathcal{D}=$ $\mathbb{R}\left[\partial_{1}, \ldots, \partial_{m}\right]$, where $\partial_{i}=\frac{\partial}{\partial c_{i}}$. It is convenient to double the degree, so we assume that deg $\partial_{i}=2$. Given any nonzero homogeneous polynomial $\Psi \in \mathbb{R}\left[c_{1}, \ldots, c_{m}\right]$ of degree $n$, consider the subspace $\operatorname{Ann}(\Psi) \subset \mathcal{D}, \operatorname{Ann}(\Psi)=\{D \in \mathcal{D} \mid D \Psi=0\}$. It is easily seen, that $\operatorname{Ann}(\Psi)$ is a graded ideal, and the quotient algebra $\mathcal{D} / \operatorname{Ann}(\Psi)$ is finite-dimensional and vanishes in degrees $>2 n$. Moreover, $\mathcal{D} / \operatorname{Ann}(\Psi)$ is a commutative Poincare duality algebra of formal dimension $2 n$ (see e.g. Timorin 1999, Prop. 2.5.1).

Now consider a complete simplicial multi-fan $\Delta$ and apply this construction to the volume polynomial $V_{\Delta}$. In result we obtain a Poincare duality algebra $\mathcal{A}^{*}(\Delta):=$ $\mathcal{D} / \operatorname{Ann}\left(V_{\Delta}\right)$ associated with a multi-fan $\Delta$. The main goal of this work is to study the volume polynomials and investigate the structure of the corresponding algebras and to show their relation to other topics in combinatorics, convex geometry, commutative algebra, and topology.

The work has the following structure. In Sects. 2 and 3 we review the basic notions of the theory of multi-fans and in Sect. 4 we review the notion of the index map which 
is the key ingredient in the construction of the volume polynomial. In the work Hattori and Masuda (2003), introducing multi-fans, the existence of a lattice $N \cong \mathbb{Z}^{n} \subset V$ was assumed, so that multi-fans are non-singular (or at least rational) with respect to this lattice. In our paper we consider general multi-fans, probably non-rational. Instead of a lattice we assume that the ambient space $V$ has a fixed inner product. This allows, in particular, to define and compute volumes of multi-polytopes in $V^{*}=V$ of dimensions smaller than $n$ (dealing with lattices, only unimodular volumes make sense). The exposition of the multi-fan theory is built to comply with this continuous setting. Nevertheless, all statements in the introductory sections follow from their lattice analogues discussed in Hattori and Masuda (2003).

In Sect. 5 we prove the basic enumerative properties of the volume polynomial. While the values of $V_{\Delta}$ are the volumes of multi-polytopes, the values of its partial derivatives are the volumes of proper faces of these multi-polytopes up to certain constants. These relations will be used further in Sect. 9.

In Sect. 6 we prove a general formula (actually, a family of formulas) for the volume polynomial, and indicate a geometrical procedure which allows to find nontrivial linear identities on the powers of linear forms. For actual convex polytopes our formula coincides with the Lawrence's formula (Lawrence 1991), which is well known in computational geometry.

In Sect. 7 we review the general correspondence between homogeneous polynomials and Poincare duality algebras, known as the Macaulay duality. Using this correspondence we obtain an algebra $\mathcal{A}^{*}(\Delta)$ as a Poincare duality algebra corresponding to the volume polynomial $V_{\Delta}$. One way to obtain this algebra is via differential operators as discussed above. Another way involves the index map of a multi-fan.

The structure of multi-fan algebras in some particular cases is described in Sect. 8. Every (complete simplicial) multi-fan has an underlying simplicial cycle. If this cycle is a homology sphere $K$, then $\mathcal{A}^{*}(\Delta)$ is the quotient of the Stanley-Reisner algebra of $K$ by a linear system of parameters, and the dimensions of its graded components are the $h$-numbers of $K$. This is similar to ordinary fans. If the underlying simplicial cycle is a homology manifold, the algebra $\mathcal{A}^{*}(\Delta)$ is the quotient of the Stanley-Reisner algebra by the linear system of parameters and by the certain ideal introduced and studied by Novik and Swartz (2009a, b). In this case the dimensions of the graded components of $\mathcal{A}^{*}(\Delta)$ are the $h^{\prime \prime}$-numbers of $K$. A short exposition of the NovikSwartz theory is provided.

Section 9 aims to generalize a classical Minkowski theorem on convex polytopes to multi-polytopes. The direct Minkowski theorem has a straightforward generalization which can be used to obtain linear relations in the algebra $\mathcal{A}^{*}(\Delta)$. On the other hand, the inverse Minkowski theorem, properly formulated, is controlled by the power map $\mathcal{A}^{2}(\Delta) \rightarrow \mathcal{A}^{2 n-2}(\Delta), a \mapsto a^{n-1}$.

In Sect. 10 we answer the question which polynomials are volume polynomials of multi-fans, and which Poincare duality algebras are algebras of multi-fans. We prove that every Poincare duality algebra generated in degree 2 is isomorphic to $\mathcal{A}^{*}(\Delta)$ for some complete simplicial multi-fan $\Delta$. This proves that the analogue of the $g$-theorem fails for multi-fans.

The basic operations on multi-fans, such as flips and connected sums, and their effects to multi-fan algebras are described in Sect. 11. In particular, we prove that, 
under flips, the dimensions of graded components of $\mathcal{A}^{*}(\Delta)$ change similarly to $h$ numbers of simplicial complexes.

Finally, in Sect. 12 we discuss the relation of $\mathcal{A}^{*}(\Delta)$ to the cohomology of torus manifolds. It is known that, for complete smooth toric variety $X$, the cohomology ring $H^{*}(X ; \mathbb{R})$ coincides with the algebra $\mathcal{A}^{*}\left(\Delta_{X}\right)$ of the corresponding fan. Situation with general torus manifolds and their multi-fans is more complicated. Nevertheless, in a certain sense, the multi-fan algebra $\mathcal{A}^{*}\left(\Delta_{X}\right)$ gives a "lower bound" for the cohomology of a torus manifold $X$.

\section{Definitions: Multi-Fans}

\subsection{Multi-Fans as Parametrized Collections of Cones}

Let us recall the definition and basic properties of multi-fans. This exposition follows the lines of Hattori and Masuda (2003).

Consider an oriented vector space $V \cong \mathbb{R}^{n}$ with a lattice $N \subset V, N \cong \mathbb{Z}^{n}$. A subset of the form $\kappa=\left\{r_{1} v_{1}+\cdots+r_{k} v_{k} \mid r_{i} \geqslant 0\right\}$ for given $v_{1}, \ldots, v_{k} \in V$ is called a cone in $V$. Dimension of $\kappa$ is the dimension of the linear hull of $\kappa$. A cone is called strongly convex if it contains no line through the origin. In the following all cones are assumed strongly convex.

Using classical construction of supporting hyperplane one can define the faces of $\kappa$, which are also the cones of smaller dimensions. If the generating set $v_{1}, \ldots, v_{k}$ may be chosen linearly independent (resp. rational, the part of basis of the lattice $N$ ), $\kappa$ is called simplicial (resp. rational, unimodular). Let Cone $(V)$ denote the set of all cones in $V$. This set obtains a partial order: $\kappa_{1} \prec \kappa_{2}$ whenever $\kappa_{1}$ is a face of $\kappa_{2}$.

Let $\Sigma$ be a finite partially ordered set with the minimal element $*$. Suppose there is a map $C: \Sigma \rightarrow \operatorname{Cone}(V)$ such that

1. $C(*)=\{0\}$;

2. If $I<J$ for $I, J \in \Sigma$, then $C(I) \prec C(J)$;

3. For any $J \in \Sigma$ the map $C$ restricted on $\{I \in \Sigma \mid I \leqslant J\}$ is an isomorphism of ordered sets onto $\{\kappa \in \operatorname{Cone}(V) \mid \kappa \preceq C(J)\}$.

The image $C(\Sigma)$ is a finite set of cones in $V$. We may think of a pair $(\Sigma, C)$ as a set of cones in $V$ labeled by the ordered set $\Sigma$.

The poset $\Sigma$ obtains a rank function: $\operatorname{rk}(I):=\operatorname{dim} C(I)$. The set of elements in $\Sigma$ having maximal rank $n$ is denoted $\Sigma^{\langle n\rangle}$.

Consider an arbitrary function $\sigma: \Sigma^{\langle n\rangle} \rightarrow\{-1,+1\}$ called a sign function.

Definition 2.1 (Old definition) The triple $\Delta:=(\Sigma, C, \sigma)$ is called a multi-fan in $V$. The number $n=\operatorname{dim} V$ is called the dimension of $\Delta$.

Multi-fan $\Delta$ is called simplicial (resp. rational, non-singular) if the values of $C$ are simplicial (resp. rational, unimodular) cones. In the following we will always assume that $\Delta$ is simplicial. Then every cone of $\Delta$ is simplicial and property (3) of the map $C$ implies that $\Sigma$ is a simplicial poset. Recall that a poset $\Sigma$ is called simplicial if any lower order ideal $\Sigma_{\leqslant J}:=\{I \in \Sigma \mid I \leqslant J\}$ is isomorphic to the poset of faces of a simplex (i.e. a boolean lattice). 


\subsection{Multi-Fans as Pairs of Weight and Characteristic Functions}

Note that Definition 2.1 of a multi-fan slightly differs from the definition of multi-fan given in Hattori and Masuda (2003). To establish the correspondence consider the following construction. Let $[m]=\{1, \ldots, m\}$ denote the set of vertices of $\Sigma$.

The signs of maximal simplices in $\Sigma$ determine two functions on $\left(\begin{array}{c}{[m]} \\ n\end{array}\right)$, the set of all $n$-subsets of $[m]$ :

$$
w^{ \pm}:\left(\begin{array}{c}
{[m]} \\
n
\end{array}\right) \rightarrow \mathbb{Z}_{\geqslant 0},
$$

where $w^{+}\left(\left\{i_{1}, \ldots, i_{n}\right\}\right)$ (resp. $\left.w^{-}\left(\left\{i_{1}, \ldots, i_{n}\right\}\right)\right)$ equals the number of simplices $I \in$ $\Sigma^{\langle n\rangle}$ on the vertices $\left\{i_{1}, \ldots, i_{n}\right\}$ having sign +1 (resp. -1 ). Although both functions $w^{+}, w^{-}$are important by topological reasons (see Hattori and Masuda 2003), only their difference $w:=w^{+}-w^{-}$is relevant to our work. So far $w$ is a function which assigns an integral number to each $n$-subset of $[m]$. Let us consider a pure simplicial complex $K$ on the set $[m]$ whose maximal simplices $K^{\langle n\rangle}$ are the subsets $I \subset[m]$ satisfying $w(I) \neq 0$. To reach greater generality we allow $w$ to take real values, thus

$$
w:\left(\begin{array}{c}
{[m]} \\
n
\end{array}\right) \rightarrow \mathbb{R} .
$$

Each vertex $i \in[m]$ corresponds to a ray (i.e. 1-dimensional cone) of $\Delta$. We choose a generator in each ray. This gives a so called characteristic function $\lambda:[m] \rightarrow V$, such that the ray $C(i)$ is generated by $\lambda(i)$ for every $i \in[m]$. It satisfies the following property:

$$
\text { if }\left\{i_{1}, \ldots, i_{k}\right\} \in K \text {, then } \lambda\left(i_{1}\right), \ldots, \lambda\left(i_{k}\right) \in V \text { are linearly independent. }
$$

This condition is called $*$-condition.

Note that in Hattori and Masuda (2003) all multi-fans were assumed rational. In this case the generator $\lambda(i)$ can be chosen canonically as a unique primitive integral vector contained in $C(i)$. Since we want to include non-rational simplicial multi-fans in our consideration, we should specify the generators somehow in order for the subsequent calculations to make sense.

Finally we get to the following definition

Definition 2.2 (New definition) A triple $(K, w, \lambda)$ is called a simplicial multi-fan in $V$. Here $w:\left(\begin{array}{c}{[m]} \\ n\end{array}\right) \rightarrow \mathbb{R}$ is a weight function, $K$ is a simplicial complex which is the support of $w$, and $\lambda:[m] \rightarrow V$ is a characteristic function. Characteristic function satisfies $*$-condition with respect to $K$ : if $I=\left\{i_{1}, \ldots, i_{k}\right\} \in K$, then the vectors $\lambda\left(i_{1}\right), \ldots, \lambda\left(i_{k}\right)$ are linearly independent in $V$.

Here $K$ may have ghost vertices, i.e. $i \in[m]$ such that $\{i\} \notin K$. The value of characteristic function in such vertices may be arbitrary (even zero). In the following we will not pay too much attention to ghost vertices since their presence does not affect the calculations. 
Strictly speaking, the new definition is not equivalent to the old one, since we cannot restore the poset $\Sigma$ and the sign function $\sigma: \Sigma^{\langle n\rangle} \rightarrow\{ \pm 1\}$ when $w$ takes non-integral values. Even in the integral case we cannot restore $\Sigma$ uniquely. On the other hand, as was shown above, every multi-fan in the sense of old definition determines a multi-fan in the sense of new definition. We will work with the new definition most of the time.

Remark 2.3 When passing from the old definition to the new one, we may lose an important information. For example consider the multi-fan in $\mathbb{R}^{2}=\left\langle e_{1}, e_{2}\right\rangle$ whose maximal cones are two copies of the non-negative cone (i.e. the cone generated by basis vectors $e_{1}, e_{2}$ ), and two rays are generated by $e_{1}$ and $e_{2}$. One of the maximal cones is taken with the sign +1 and the other with the sign -1 . We remark that such multi-fan corresponds to the torus manifold $S^{4}$ (Hattori and Masuda 2003). We have $w^{+}(\{1,2\})=w^{-}(\{1,2\})=1$, therefore $w(\{1,2\})=0$. Thus $K$ is empty (equivalently, $w:\left(\begin{array}{c}{[\mathrm{m}]} \\ n\end{array}\right) \rightarrow \mathbb{R}$ vanishes).

One way to avoid such situations is to assume in the beginning that $\Sigma$ itself is a simplicial complex rather than a general simplicial poset. In this case $K$ coincides with $\Sigma$ and the weight function $w$ on $K$ coincides with the sign function $\sigma$. In particular, $w$ takes the value \pm 1 on each maximal simplex of $K$ (see Example 2.9 below).

\subsection{Underlying Simplicial Chain}

Let $\triangle_{[m]}$ denote an abstract simplex on the vertex set $[m]$, and let $\triangle_{[m]}^{(n-1)}$ be its $(n-1)$ skeleton. Every subset $I \subset[m],|I|=n$ may be considered as a maximal simplex of $\triangle_{[m]}^{(n-1)}$. If $I \in K^{\langle n\rangle}$, then we can orient $I$ as follows: we say that the order of vertices $\left(i_{1}, \ldots, i_{n}\right)$ of $I$ is positive if and only if the basis $\left(\lambda\left(i_{1}\right), \ldots, \lambda\left(i_{n}\right)\right)$ determines the positive orientation of $V$.

Definition 2.4 The element

$$
w_{c h}=\sum_{I \in K^{\langle n\rangle}} w(I) I \in C_{n-1}(K ; \mathbb{R}) \subseteq C_{n-1}\left(\triangle_{[m]}^{(n-1)} ; \mathbb{R}\right)
$$

is called the underlying chain of a multi-fan $\Delta$. Here $C_{n-1}(K ; \mathbb{R})$ denotes the group of simplicial chains of $K$.

\subsection{Complete Multi-Fans}

Let us briefly recall the notion of projected multi-fan. We give the construction in terms of new definition of multi-fan although the similar construction may be given in terms of simplicial posets and sign functions.

Let $\Delta=(K, w, \lambda)$ be a simplicial multi-fan in the space $V$, and let $I=$ $\left\{i_{1}, \ldots, i_{k}\right\} \in K$ be a simplex. Let $V_{I}$ denote the quotient vector space $V /\left\langle\lambda\left(i_{1}\right), \ldots\right.$, $\left.\lambda\left(i_{k}\right)\right\rangle$. Consider the multi-fan $\Delta_{I}=\left(\mathrm{lk}_{K} I, w_{I}, \lambda_{I}\right)$ in $V_{I}$ defined as follows:

$-\mathrm{lk}_{K} I:=\{J \subset[m] \backslash I \mid I \cup J \in K\}$ is the link of the simplex $I$ in $K$.

$-w_{I}(J):=w(I \cup J)$ for every $J \in \operatorname{lk}_{K} I,|J|=n-|I|$. 
$-\lambda_{I}(j)$ is the image of $\lambda(j) \in V$ under the natural projection $V \rightarrow V_{I}=$ $V /\left\langle\lambda\left(i_{1}\right), \ldots, \lambda\left(i_{k}\right)\right\rangle$. It is easily seen that $\lambda_{I}$ satisfies $*$-condition.

If we choose some orientation of a simplex $I \in K$, the space $V_{I}$ obtains an orientation induced from $V$. To be precise, let us say that the basis $\left(\left[v_{1}\right], \ldots,\left[v_{n-k}\right]\right)$ determines a positive orientation of $V_{I}$ if the basis $\left(v_{1}, \ldots, v_{n-k}, \lambda\left(i_{1}\right), \ldots, \lambda\left(i_{k}\right)\right)$ is a positive basis of $V$ for a chosen positive order $\left(i_{1}, \ldots, i_{k}\right)$ of vertices of $I$.

We call $\Delta_{I}$ the projected multi-fan of $\Delta$. The construction satisfies the hereditary relation $\left(\Delta_{I_{1}}\right)_{I_{2}}=\Delta_{I_{1} \sqcup I_{2}}$ whenever it makes sense, and there holds $\Delta \varnothing=\Delta$.

Let us call a vector $v \in V$ generic with respect to $\Delta$ if it is not contained in the vector subspaces spanned by the cones of $\Delta$ of dimensions $<n$. For any such $v$ define the number $d_{v}=\sum w(I) \in \mathbb{R}$, where the sum is taken over all subsets $I=\left\{i_{1}, \ldots, i_{n}\right\} \subset[m]$ such that the cone generated by $\lambda\left(i_{1}\right), \ldots, \lambda\left(i_{n}\right)$ contains $v$.

Definition 2.5 The multi-fan $\Delta$ is called pre-complete if $d_{v}$ does not depend on a generic vector $v \in V$. In this case $d_{v}$ is called the degree of $\Delta$. The multi-fan $\Delta$ is called complete if the projected multi-fan $\Delta_{I}$ is pre-complete for any simplex $I \in K$.

Remark 2.6 Note that this definition allows $w$ to be constantly zero. We call a multifan zero if its weight function is constantly zero. A zero multi-fan is pre-complete and therefore complete.

Proposition 2.7 A multi-fan $\Delta$ is complete if and only if its underlying simplicial chain $w_{c h} \in C_{n-1}\left(\triangle_{[m]}^{(n-1)} ; \mathbb{R}\right)$ is a cycle, that is $d w_{c h}=0$ for the standard simplicial differential $d: C_{n-1}\left(\triangle_{[m]}^{(n-1)} ; \mathbb{R}\right) \rightarrow C_{n-2}\left(\triangle_{[m]}^{(n-1)} ; \mathbb{R}\right.$ ) (if $n=1$, we assume that $d: C_{0}\left(\triangle_{[m]}^{(n-1)} ; \mathbb{R}\right) \rightarrow \mathbb{R}$ is the augmentation map $)$.

Proof In the case when $w$ takes only integral values, the statement is proved in Hattori and Masuda (2003, Sec. 6). If $w$ takes only rational values, scaling the values of $w$ by a common denominator reduces the task to the integral case. It remains to prove the statement for real-valued $w$. Both conditions " $\Delta$ is complete" and " $d w_{c h}=0$ " determine rational vector subspaces in the space of all possible weight functions (it is not difficult to define the pre-completeness condition in terms of the "wall-crossing relations", which are linear relations on $w(I)$ with integral coefficients). Thus the rational case implies the real case.

For convenience we summarize the discussion by the following definition.

Definition 2.8 (Complete simplicial multi-fan) A complete simplicial multi-fan is a pair $\left(w_{c h}, \lambda\right)$, where $w_{c h}=\sum_{I \subset[m],|I|=n} w(I) I \in Z_{n-1}\left(\triangle_{[m]}^{(n-1)}\right)$ is a simplicial cycle on $m$ vertices, and $\lambda:[m] \rightarrow V$ is any function satisfying the condition: $\{\lambda(i)\}_{i \in I}$ is a basis of $V$ if $|I|=n$ and $w(I) \neq 0$.

For a complete multi-fan $\Delta$ the corresponding homology class $\left[w_{c h}\right] \in \widetilde{H}_{n-1}(K ; \mathbb{R})$ $\subset \widetilde{H}_{n-1}\left(\triangle_{[m]}^{(n-1)} ; \mathbb{R}\right)$ will be denoted $[\Delta]$ and called the underlying homology class of $\Delta$. Since $C_{n}\left(\triangle_{[m]}^{(n-1)} ; \mathbb{R}\right)=0$, the groups $Z_{n-1}\left(\triangle_{[m]}^{(n-1)}\right)$ and $\widetilde{H}_{n-1}\left(\triangle_{[m]}^{(n-1)}\right)$ may be identified. Thus $w_{c h}$ and $[\Delta]$ are just two different notations for the same object. 
Example 2.9 A pseudomanifold is a pure simplicial complex such that each simplex of codimension one is contained in exactly two maximal simplices. It is called orientable if all its maximal simplices can be simultaneously oriented so that orientations of neighboring simplices are compatible. One obvious way to obtain a complete multifan is to start with any oriented pseudomanifold $K$ of dimension $n-1$ on the set of vertices $[m]$, and take any characteristic function $\lambda:[m] \rightarrow V$. Since $K$ is oriented, every maximal simplex $I$ of $K$ becomes oriented, but this orientation may be different from the one determined by characteristic function (see Sect. 2.3). Let $w(I)$ be +1 or -1 depending on whether these two orientations agree or not. Let us extend the weight function by zeroes to non-simplices of $K$. The corresponding simplicial chain $w_{c h}=\sum_{I} w(I) I \in C_{n-1}\left(\triangle_{[m]}^{(n-1)} ; \mathbb{R}\right)$ is closed, since it is exactly the fundamental chain of $K$ in $\triangle_{[m]}^{(n-1)}$. Therefore, $\left(w_{c h}, \lambda\right)$ is a complete simplicial fan.

Example 2.10 The previous example may be restricted to the case when $K$ is a homology sphere or homology manifold. We will study these two cases in more detail in Sect. 8.

We say that $\Delta$ is based on an orientable simplicial pseudomanifold $K$ if the corresponding simplicial cycle is given by $K$.

There is one interesting feature of (complete) multi-fans revealed by Definitions 2.2 and 2.8. The multi-fans with the given set of vertices $[m]$ and the given characteristic function $\lambda$ form a vector space: we may add them by adding their weights and multiply by real numbers by scaling their weights. Let MultiFans ${ }_{\lambda}$ denote the vector space of complete multi-fans with the given characteristic function $\lambda$. This space may be identified with certain vector subspace of $Z_{n-1}\left(\triangle_{[m]}^{(n-1)} ; \mathbb{R}\right)$. We will discuss this subspace in detail in Sect. 10.3. The set of multi-fans with integral weights forms a lattice inside MultiFans $\lambda$ which is a certain sublattice of $Z_{n-1}\left(\triangle_{[m]}^{(n-1)} ; \mathbb{Z}\right)$.

\section{Definitions: Multi-Polytopes}

\subsection{Multi-Polytopes}

Let $\Delta$ be a simplicial multi-fan with characteristic function $\lambda:[m] \rightarrow V$. Let $H P\left(V^{*}\right)$ denote the set of all affine hyperplanes in the dual vector space $V^{*}$.

For each $i \in[m]$ choose an affine hyperplane $\mathcal{H}(i) \subset V^{*}$ in the dual space which is orthogonal to the linear hull of the $i$-th cone. In other words, $\mathcal{H}(i)$ is defined by equation $\mathcal{H}(i)=\left\{u \in V^{*} \mid\langle u, \lambda(i)\rangle=c_{i}\right\}$ for some constant $c_{i} \in \mathbb{R}$ called the support parameter of $\mathcal{H}(i)$.

Definition 3.1 $A$ multi-polytope $P$ is a pair $(\Delta, \mathcal{H})$, where $\Delta$ is a multi-fan, and $\mathcal{H}:[m] \rightarrow H P\left(V^{*}\right)$ is a function such that $\mathcal{H}(i)$ is orthogonal to $\lambda(i)$ for any $i \in[m]$. We say that $P$ is based on the multi-fan $\Delta$.

Although the definition may be stated in general, we restrict to simplicial multi-fans $\Delta$, in which case $P$ is called a simple multi-polytope. 
Let us denote the set of all multi-polytopes based on $\Delta$ by $\operatorname{Poly}(\Delta)$. Every such multi-polytope is completely determined by its support parameters $c_{1}, \ldots, c_{m}$. Thus $\operatorname{Poly}(\Delta)$ has natural coordinates $\left(c_{1}, \ldots, c_{m}\right)$ and may be identified with $\mathbb{R}^{m}$. This space is called the space of analogous multi-polytopes based on $\Delta$.

To simplify notation, we denote $\mathcal{H}(i)$ by $H_{i}$ and set

$$
H_{I}:=\bigcap_{i \in I} H_{i} \quad \text { for } I \in K
$$

$H_{I}$ is a codimension $|I|$ affine subspace in $V^{*}$, since the normals of the hyperplanes $H_{i}, i \in I$ are linearly independent by $*$-condition. In particular, when $I$ is a maximal simplex, $I \in K^{\langle n\rangle}, H_{I}$ is a point in $V^{*}$ which is called the vertex of $P$.

Definition 3.2 Let $\Delta$ be a simplicial multi-fan in $V$ with the underlying simplicial complex $K$ and let $P$ be a simple multi-polytope based on $\Delta$. Let $I \in K$. Consider a simple multi-polytope $F_{I}=\left(\Delta_{I}, \mathcal{H}_{I}\right)$ in the space $H_{I} \subset V^{*}$. Note that the projected multi-fan $\Delta_{I}$ is defined in the space $V_{I}$ (see Sect. 2.4), so the multi-polytope based on $\Delta_{I}$ should formally lie in $V_{I}^{*}$. Nevertheless, we may identify $H_{I}$ with $V_{I}^{*}$. The supporting hyperplanes of $F_{I}$ are defined as follows: $\mathcal{H}_{I}(j)=H_{I} \cap H_{j}$ for any vertex $j$ of $\operatorname{lk}_{K} I$. The multi-polytope $F_{I}$ is called the face of $P$ dual to $I$.

\subsection{Duistermaat-Heckman Function of a Multi-Polytope}

Suppose $I \in K^{\langle n\rangle}$. Then the set $\{\lambda(i) \mid i \in I\}$ is a basis of $V$. Denote its dual basis of $V^{*}$ by $\left\{u_{i}^{I} \mid i \in I\right\}$, i.e. $\left\langle u_{i}^{I}, \lambda(j)\right\rangle=\delta_{i j}$ where $\delta_{i j}$ denotes the Kronecker delta. Take a generic vector $v \in V$. Then $\left\langle u_{i}^{I}, v\right\rangle \neq 0$ for all $I \in K^{\langle n\rangle}$ and $i \in I$. Set

$$
(-1)^{I}:=(-1)^{\sharp\left\{i \in I \mid\left\langle u_{i}^{I}, v\right\rangle>0\right\}} \text { and }\left(u_{i}^{I}\right)^{+}:= \begin{cases}u_{i}^{I} & \text { if }\left\langle u_{i}^{I}, v\right\rangle>0, \\ -u_{i}^{I} & \text { if }\left\langle u_{i}^{I}, v\right\rangle<0 .\end{cases}
$$

We denote by $C^{*}(I)^{+}$the cone in $V^{*}$ spanned by $\left(u_{i}^{I}\right)^{+}$'s $(i \in I)$ with apex at a vertex $H_{I}$ of a multi-polytope $P$, and by $\phi_{I}$ the function on $V^{*}$ which takes value 1 inside $C^{*}(I)^{+}$and 0 outside (the indicator function of the cone $C^{*}(I)^{+}$).

Definition 3.3 A function $\mathrm{DH}_{P}$ on $V^{*} \backslash \bigcup_{i=1}^{m} H_{i}$ defined by

$$
\sum_{I \in K}(-1)^{I} w(I) \phi_{I}
$$

is called a Duistermaat-Heckman function associated with $P$.

The summands in the definition depend on the choice of a generic vector $v \in V$. Nevertheless, the function itself is independent of $v$ when $\Delta$ is complete (we refer to Hattori and Masuda (2003) when $w$ is integral-valued and note that the same argument works for real weights). 
The function $\mathrm{DH}_{P}$ for a simple multi-polytope $P$ based on a complete multi-fan has the following geometrical interpretation. Let $S$ be the realization of first barycentric subdivision of $K$ and let $G_{I} \subset S$ be the dual face of $I \in K, I \neq \varnothing$, i.e. a realization of the set $\left\{\left\{I<I_{1}<\cdots<I_{k}\right\} \in K^{\prime}\right\}$. If $I \in K^{\langle n\rangle}$, then $G_{I}$ is a point. For a given multi-polytope $P$ based on $\Delta$ there exists a continuous map $\psi: S \rightarrow V^{*}$ such that $\psi\left(G_{I}\right) \subset H_{I}$ for any $I \in K, I \neq \varnothing$ (in particular, when $I \in K^{\langle n\rangle}$, this map sends the point $G_{I} \in S$ to the vertex $H_{I}$ of a multi-polytope $P$ ). This map is unique up to homotopy preserving the stratifications.

Let us take any point $u \in V^{*} \backslash \bigcup_{i=1}^{m} H_{i}$. Then $u$ is not contained in the image of $\psi$ by the construction of $\psi$. Thus we may consider the induced map in homology:

$$
\psi_{*}: \widetilde{H}_{n-1}(S ; \mathbb{R}) \rightarrow \widetilde{H}_{n-1}\left(V^{*} \backslash\{u\} ; \mathbb{R}\right) .
$$

The underlying simplicial cycle $[\Delta]$ may be considered as an element of the group $\widetilde{H}_{n-1}(S ; \mathbb{R})$. Since $V^{*}$ is oriented, we have the fundamental class $\left[V^{*} \backslash\{u\}\right] \in$ $\widetilde{H}_{n-1}\left(V^{*} \backslash\{u\} ; \mathbb{R}\right)$. Thus

$$
\psi_{*}([\Delta])=\mathrm{WN}_{P}(u) \cdot\left[V^{*} \backslash\{u\}\right],
$$

for some number $\mathrm{WN}_{P}(u) \in \mathbb{R}$. This number has a natural meaning of winding number of cycle $[\Delta]$ around $u$. It happens that this number is exactly the value of $\mathrm{DH}_{P}$ at the point $u \in V^{*}$ (see details in Hattori and Masuda 2003, Sec. 6).

It is easily seen from the above consideration that $\mathrm{DH}_{P}$ has a compact support when $\Delta$ is complete. Thus in the case of complete multi-fan we may define the volume of a multi-polytope $P$ as

$$
\operatorname{Vol}(P)=\int_{V^{*}} \operatorname{DH}_{P}(u) d \mu
$$

with respect to some Euclidean measure on $V^{*}$ (in a presence of a lattice $N \subset V$ the measure is normalized so that the fundamental domain of $N^{*} \subset V^{*}$ has volume 1).

Finally, we may consider the volume as a function on the space $\operatorname{Poly}(\Delta) \cong \mathbb{R}^{m}$ of analogous multi-polytopes. We have a function $V_{\Delta}: \mathbb{R}^{m} \rightarrow \mathbb{R}$ whose value at $\left(c_{1}, \ldots, c_{m}\right)$ equals $\operatorname{Vol}(P)$ for the multi-polytope $P$ with the support parameters $c_{1}, \ldots, c_{m}$. The goal of the next section is to study this function using equivariant localization ideas and prove Theorem 1.1.

Remark 3.4 Needless to say that in case of actual simple convex polytopes the notions introduced above coincide with the classical ones. If $P$ is a simple convex polytope and $\Delta$ is its normal fan, then $\mathrm{DH}_{P}$ takes the value 1 inside $P$ and 0 outside. The volume of $P$ is just the usual volume. Note that even if $\Delta$ is an actual fan, not all multi-polytopes based on $\Delta$ are actual convex polytopes. Nevertheless, the notion of volume and Duistermaat-Heckman function have transparent geometrical meanings for all of them.

Example 3.5 Consider the two-dimensional multi-fan $\Delta$ with $m=5$ and $V=\mathbb{R}^{2}$ depicted on Fig. 1, left. Its characteristic function is the following: $\lambda(1)=(1,0)$, 
$\Delta$

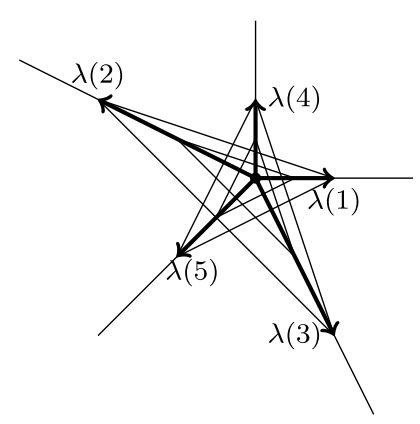

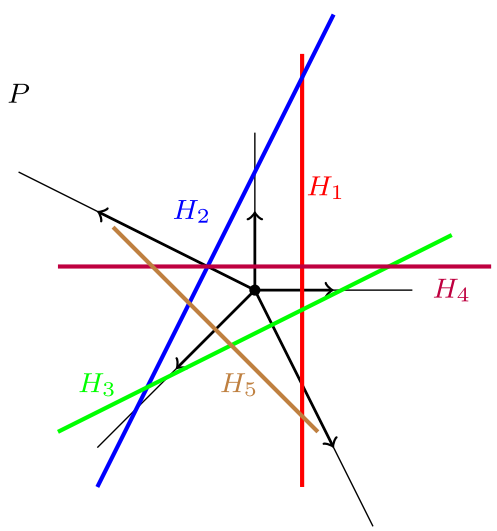

Fig. 1 Example of a multi-fan $\Delta$ and a multi-polytope $P$ based on it

Fig. 2 Duistermaat-Heckman function of the multi-polytope $P$

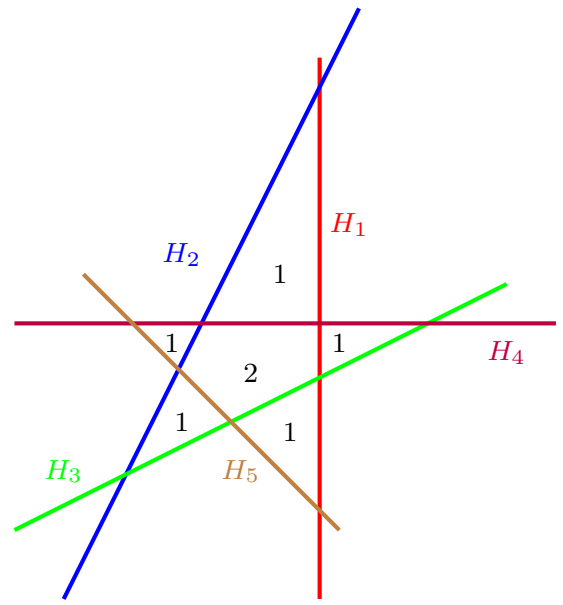

$\lambda(2)=(-2,1), \lambda(3)=(1,-2), \lambda(4)=(0,1), \lambda(5)=(-1,-1)$. The weight function takes the value 1 on the subsets $\{1,2\},\{2,3\},\{3,4\},\{4,5\},\{1,5\}$ and the value 0 on all other subsets. Geometrically this indicates the fact that in the multi-fan we have the cones generated by $\{\lambda(1), \lambda(2)\},\{\lambda(2), \lambda(3)\}$, etc. with multiplicity one, and do not have the cones generated by $\{\lambda(1), \lambda(3)\},\{\lambda(1), \lambda(4)\}$, and so on. It can be seen that every generic point of $V=\mathbb{R}^{2}$ is covered by exactly two cones, therefore $\Delta$ is pre-complete of degree 2 . Moreover, a simple check shows that all its projected multi-fans are complete. Hence $\Delta$ is complete. The underlying chain of $\Delta$ has the form $(1,2)+(2,3)+(3,4)+(4,5)+(5,1) \in C_{1}\left(\triangle_{[5]}^{(1)} ; \mathbb{R}\right)$ which is obviously a simplicial cycle. The underlying complex $K$ of $\Delta$ is a circle made of 5 segments, and $[\Delta]$ is its fundamental class.

An example of a multi-polytope $P$ based on $\Delta$ is shown at Fig. 1, right. Each hyperplane $H_{i}$ is orthogonal to the linear span of the corresponding ray $\lambda(i)$ of $\Delta$, $i \in[5]$. The Duistermaat-Heckman function of $P$ is shown on Fig. 2. The function 
is constant on the chambers: it takes value 2 in the middle pentagon since the multipolytope "winds" around the points of this region twice, and takes value 1 on triangles adjacent to the central pentagon. The value of $\mathrm{DH}_{P}$ in all other chambers is 0 . The volume of a multi-polytope is therefore not just the volume of the five-point star: the points in the central region contribute to the volume twice.

\section{Volume Polynomial from the Index Map}

\subsection{Index Map}

Let $\Delta=\left(w_{c h}, \lambda\right)$ be a simplicial multi-fan in $V \cong \mathbb{R}^{n}$ with $m$ rays. The characteristic function $\lambda:[m] \rightarrow V$ may be considered as a linear map $\lambda: \mathbb{R}^{m} \rightarrow V$ which sends the basis vector $e_{i} \in \mathbb{R}^{m}, i \in[m]$ to $\lambda(i)$. Let $\left\{x_{i}\right\}_{i \in[m]}$ be the basis of $\left(\mathbb{R}^{m}\right)^{*}$ dual to $\left\{e_{i}\right\}_{i \in[m]}$, so that $\left(\mathbb{R}^{m}\right)^{*}=\left\langle x_{1}, \ldots, x_{m}\right\rangle$. Let us also consider the adjoint map $\lambda^{\top}: V^{*} \rightarrow\left(\mathbb{R}^{m}\right)^{*}$. By definition it sends the vector $u \in V^{*}$ to

$$
\sum_{i=1}^{m}\langle u, \lambda(i)\rangle x_{i}
$$

For any maximal simplex $I=\left\{i_{1}, \ldots, i_{n}\right\} \in K^{\langle n\rangle}$ the vectors $\{\lambda(i)\}_{i \in I}$ form a basis of $V$ according to $*$-condition, defined in Sect. 2.2. Let $\left\{u_{i}^{I}\right\}_{i \in I}$ be the dual basis of $V^{*}$. Let $\iota_{I}:\left(\mathbb{R}^{m}\right)^{*} \rightarrow V^{*}$ be the linear map defined by

$$
\iota_{I}\left(x_{i}\right)= \begin{cases}u_{i}^{I} & \text { if } i \in I, \\ 0, & \text { if } i \notin K .\end{cases}
$$

Consider $\mathbb{R}\left[x_{1}, \ldots, x_{m}\right]$, the algebra of polynomials on $\left(\mathbb{R}^{m}\right)^{*}$. Also let $\mathbb{R}\left[V^{*}\right]$ denote the algebra of polynomials on $V^{*}$. Both polynomial algebras are graded, where we set the degrees of the generating spaces $\left(\mathbb{R}^{m}\right)^{*}$ and $V^{*}$ to 2 . The linear map $\iota_{I}$ induces the graded algebra homomorphism

$$
\iota_{I}: \mathbb{R}\left[x_{1}, \ldots, x_{m}\right] \rightarrow \mathbb{R}\left[V^{*}\right],
$$

denoted by the same letter. In the following, if $A$ is a graded algebra, we denote by $A_{j}$ its homogeneous part of degree $j$.

Let $S^{-1} \mathbb{R}\left[V^{*}\right]$ denote the ring of rational functions over $\mathbb{R}\left[V^{*}\right]$ graded in a natural way. Given a weight function $w: K^{\langle n\rangle} \rightarrow \mathbb{R}$ we can define the linear map $\pi_{!}^{\Delta}: \mathbb{R}\left[x_{1}, \ldots, x_{m}\right] \rightarrow S^{-1} \mathbb{R}\left[V^{*}\right]$ as the following weighted sum:

$$
\pi_{!}^{\Delta}(x)=\sum_{I \in K^{\langle n\rangle}} \frac{w(I) \iota_{I}(x)}{\left|\operatorname{det} \lambda_{I}\right| \prod_{i \in I} \iota_{I}\left(x_{i}\right)}
$$

for $x \in \mathbb{R}\left[x_{1}, \ldots, x_{m}\right]$. We assume that an inner product is fixed on $V$, so that $\left|\operatorname{det} \lambda_{I}\right|=\left|\operatorname{det}\left(\lambda(i)_{i \in I}\right)\right|$ is well-defined even if there is no lattice in $V$. The inner 
product on $V$ induces a Euclidean measure on $V^{*}$ and $\left|\operatorname{det} \lambda_{I}\right|$ is the volume of the parallelepiped spanned by $\{\lambda(i)\}_{i \in I}$. The translation invariant measure on $V^{*}$ is assumed the same as in (3.1). The map $\pi_{1}^{\Delta}$ is well-defined since $\lambda_{I}$ are isomorphisms. It can be seen that $\pi_{!}^{\Delta}$ is homogeneous of degree $-2 n$. It is called the index map of a multi-fan $\Delta=(K, w, \lambda)$.

\section{Theorem 4.1 The following properties of $\Delta$ are equivalent:}

1. The image of $\pi_{!}^{\Delta}$ lies in $\mathbb{R}\left[V^{*}\right] \subset S^{-1} \mathbb{R}\left[V^{*}\right]$;

2. The underlying chain $w_{c h}=\sum_{I \in K^{\langle n\rangle}} w(I) I$ is closed;

3. The multi-fan $\Delta=\left(w_{c h}, \lambda\right)$ is complete.

Proof Equivalence of (2) and (3) was already shown in Proposition 2.7. The implication $(2) \Rightarrow(1)$, in case when $\lambda$ takes values in the lattice and $w$ is integer-valued, is proved in Hattori and Masuda (2003, Lm. 8.4). It should be noted that in this case $\left|\operatorname{det} \lambda_{I}\right|$ appearing in the denominator is nothing but the order of the finite group $G_{I}=N / N_{I}$, where $N \subset V$ is the lattice and $N_{I}$ is a sublattice generated by $\{\lambda(i)\}_{i \in I}$. The situation when $\lambda$ and $w$ are rational is reduced to the integral case by multiplying all values of $\lambda$ and $w$ by a common denominator (both conditions (1) and (2) are invariant under rescaling). The real case follows by continuity. Indeed, the subset of simplicial cycles with rational coefficients, $Z_{n-1}(K ; \mathbb{Q})$, is dense in $Z_{n-1}(K ; \mathbb{R})$; the right hand side of (4.2) is continuous with respect to $\lambda$ and $w$; and the subset $\mathbb{R}\left[V^{*}\right]$ is closed in $S^{-1} \mathbb{R}\left[V^{*}\right]$. Therefore, arbitrary complete multi-fan $(w, \lambda)$ can be approximated by a sequence of rational complete multi-fans $\Delta_{\alpha}=\left(w_{\alpha}, \lambda_{\alpha}\right)$ which implies that the values of $\pi_{!}^{\Delta}$ are approximated by the values of $\pi_{!}^{\Delta_{\alpha}}$. Since the values of $\pi_{!}^{\Delta_{\alpha}}$ are polynomials, so are the values of $\pi_{!}^{\Delta}$.

Let us prove that (1) implies (2). Take any simplex $J \in K$ such that $|J|=n-1$ and consider the monomial $x_{J}=\prod_{i \in J} x_{j}$ of degree $2(n-1)$ lying in $\mathbb{R}\left[x_{1}, \ldots, x_{m}\right]$. The map $\pi_{!}^{\Delta}$ lowers the degree by $2 n$ thus we have deg $\pi_{!}^{\Delta}\left(x_{J}\right)=-2$. Condition (1) implies that $\pi_{!}^{\Delta}\left(x_{J}\right)$ is a polynomial, thus $\pi_{!}^{\Delta}\left(x_{J}\right)=0$. By definition, we have

$$
\pi_{!}^{\Delta}\left(x_{J}\right)=\sum_{I \in K^{\langle n\rangle}} \frac{w(I) \iota_{I}\left(x_{J}\right)}{\left|\operatorname{det} \lambda_{I}\right| \prod_{i \in I} \iota_{I}\left(x_{i}\right)}
$$

Note that $\iota_{I}$ is a ring homomorphism and $\iota_{I}\left(x_{i}\right)=0$ if $i \notin I$ by (4.1). Therefore,

$$
\pi_{!}^{\Delta}\left(x_{J}\right)=\sum_{I \in K^{\langle n\rangle}, J \subset I} \frac{w(I) \prod_{i \in J} \iota_{I}\left(x_{i}\right)}{\left|\operatorname{det} \lambda_{I}\right| \prod_{i \in I} \iota_{I}\left(x_{i}\right)}=\sum_{j \in[m] \backslash J, I:=J \cup\{j\} \in K^{\langle n\rangle}} \frac{w(I)}{\left|\operatorname{det} \lambda_{I}\right| \iota_{I}\left(x_{j}\right)}
$$

Recall that $\iota_{I}\left(x_{i}\right)=u_{i}^{I}$, where $\left\{u_{i}^{I}\right\}_{i \in I}$ is the basis of $V^{*}$ dual to the basis $\{\lambda(i)\}_{i \in I}$ of $V$. Consider the linear functional $\varrho \in V^{*}$ taking the value $\varrho(v)=\operatorname{det}\left((\lambda(i))_{i \in J}, v\right)$ for any $v \in V$. It can be seen that $\left|\operatorname{det} \lambda_{I}\right| \iota_{I}\left(x_{j}\right) \in V^{*}$, where $I=J \cup\{j\}$, coincides with $\varrho$ up to sign. More precisely $\left|\operatorname{det} \lambda_{I}\right| \iota_{I}\left(x_{j}\right)=[I: J] \varrho$, where $[I: J]$ is the incidence sign of two simplices of $K$ (it appears because we need to permute the vectors $\left((\lambda(i))_{i \in J}, \lambda(j)\right)$ in order to get the positive determinant). Therefore, 


$$
0=\pi_{!}^{\Delta}\left(x_{J}\right)=\frac{1}{\varrho} \sum_{I \in K^{\langle n\rangle}, J \subset I}[I: J] w(I)
$$

It remains to notice that the sum in this expression is exactly the coefficient of $J$ in the simplicial chain $d w_{c h} \in C_{n-2}(K ; \mathbb{R})$. This calculation applies to any $J \in K$, $|J|=n-2$, therefore $d w_{c h}=0$.

The map $\lambda^{\top}: V^{*} \rightarrow\left(\mathbb{R}^{m}\right)^{*}$, the adjoint of $\lambda$, induces the ring homomorphism $\mathbb{R}\left[V^{*}\right] \rightarrow \mathbb{R}\left[x_{1}, \ldots, x_{m}\right]$. Hence $\mathbb{R}\left[x_{1}, \ldots, x_{m}\right]$ obtains the structure of $\mathbb{R}\left[V^{*}\right]$ module. It can be checked that $\lambda^{\top}$ is the right inverse of each $\iota_{I}:\left(\mathbb{R}^{m}\right)^{*} \rightarrow V^{*}$, therefore all ring homomorphisms $\iota_{I}: \mathbb{R}\left[x_{1}, \ldots, x_{m}\right] \rightarrow \mathbb{R}\left[V^{*}\right]$ are the $\mathbb{R}\left[V^{*}\right]$ module homomorphisms. Thus $\pi_{!}^{\Delta}$ is also a homomorphism of $\mathbb{R}\left[V^{*}\right]$-modules (even in the case $w_{c h}$ is not closed).

Remark 4.2 Note that conditions (1) and (2) in Theorem 4.1 make sense over an arbitrary field $\mathbb{k}$. We may start with a $\mathbb{k}$-valued chain $w_{c h} \in C_{n-1}(K ; \mathbb{k})$ and a characteristic function valued in $\mathbb{k}^{n}$. These data allow to define the maps $\iota_{I}$ and $\pi_{!}^{\Delta}$ absolutely similar to the real case.

Problem 4.3 Does equivalence of (1) and (2) in Theorem 4.1 hold for arbitrary fields?

For general fields we cannot reduce the task to the integral case but it is likely that there exists a straightforward algebraical proof.

\subsection{Stanley-Reisner Rings}

Let us recall the definition of the Stanley-Reisner ring.

Definition 4.4 Let $K$ be a simplicial complex on the vertex set $[m]$ and $\mathbb{k}$ be a ground ring (either $\mathbb{Z}$ or a field). The Stanley-Reisner ring is the quotient of a polynomial ring by the Stanley-Reisner ideal:

$$
\mathbb{k}[K]:=\mathbb{k}\left[x_{1}, \ldots, x_{m}\right] / I_{S R}, \quad \text { where } I_{S R}=\left(x_{i_{1}} \cdot \ldots \cdot x_{i_{k}} \mid\left\{i_{1}, \ldots, i_{k}\right\} \notin K\right),
$$

endowed with the grading $\operatorname{deg} x_{i}=2$ and the natural structure of graded $\mathbb{k}\left[x_{1}, \ldots, x_{m}\right]$-module.

For now let us concentrate on the case $\mathbb{k}=\mathbb{R}$. Given a characteristic function $\lambda$ on $K$ we may define a certain ideal in $\mathbb{R}[K]$ generated by linear forms. As before, let $\lambda^{\top}: V^{*} \rightarrow\left(\mathbb{R}^{m}\right)^{*}=\left\langle x_{1}, \ldots, x_{m}\right\rangle$ denote the adjoint map of $\lambda: \mathbb{R}^{m} \rightarrow V$. Let $\Theta$ denote the ideal of $\mathbb{R}\left[x_{1}, \ldots, x_{m}\right]$ generated by the image of $\lambda^{\top}$. By abuse of notation we denote the corresponding ideal in $\mathbb{R}[K]$ with the same letter $\Theta$.

Let us state things in the coordinate form. Fix a basis $f_{1}, \ldots, f_{n}$ of $V$. Then every characteristic value $\lambda(i), i \in[m]$ is written as a row-vector $\left(\lambda_{i, 1}, \ldots, \lambda_{i, n}\right)$, where $\lambda_{i, j} \in \mathbb{R}$. The $*$-condition for $\lambda$ (see Sect. 2.2) states that the square matrix formed by row-vectors $\left(\lambda_{i, 1}, \ldots, \lambda_{i, n}\right)_{i \in I}$ is non-degenerate for any $I \in K^{\langle n\rangle}$. 
If we consider the dual basis $\bar{f}_{1}, \ldots, \bar{f}_{n}$ in the dual space $V^{*}$, then its image under $\lambda^{\top}: V^{*} \rightarrow\left(\mathbb{R}^{m}\right)^{*}=\left\langle x_{1}, \ldots, x_{m}\right\rangle$ has the form

$$
\theta_{j}:=\lambda^{\top}\left(\bar{f}_{j}\right)=\lambda_{1, j} x_{1}+\lambda_{2, j} x_{2}+\cdots+\lambda_{m, j} x_{m}
$$

for $j=1, \ldots, n$. Thus $\Theta$ (as an ideal either in $\mathbb{R}\left[x_{1}, \ldots, x_{m}\right]$ or $\mathbb{R}[K]$ ) is generated by the elements $\theta_{1}, \ldots, \theta_{n}$. In particular, if $\lambda$ is integer-valued, then $\Theta=\left(\theta_{1}, \ldots, \theta_{n}\right)$ may be considered as a well-defined ideal in $\mathbb{Z}[K]$ or $\mathbb{Z}\left[x_{1}, \ldots, x_{m}\right]$.

It is known that the Krull dimension of $\mathbb{R}[K]$ equals $\operatorname{dim} K+1=n$ (see e.g. Stanley 1996), and $\theta_{1}, \ldots, \theta_{n}$ is a linear system of parameters in $\mathbb{R}[K]$ for any characteristic function $\lambda$ and every choice of a basis in $V$ (e.g. Buchstaber and Panov 2015, Lm. 3.3.2). Thus $\mathbb{R}[K] / \Theta$ has Krull dimension 0 , which in our case is equivalent to saying that $\mathbb{R}[K] / \Theta$ is a finite-dimensional vector space. Moreover, it is known (see e.g. Hattori and Masuda 2003, Lm. 8.1 or Ayzenberg 2016, Lm. 3.5) that the classes of monomials $x_{I}=x_{i_{1}} \cdot \ldots \cdot x_{i_{k}}$ taken for each simplex $I=\left\{i_{1}, \ldots, i_{k}\right\} \in K$ linearly span $\mathbb{R}[K] / \Theta$ (however there exist relations on these classes!).

We introduce the following notation to make the exposition consistent with that of Hattori and Masuda (2003):

$$
H_{T}^{*}(\Delta ; \mathbb{k}):=\mathbb{k}[K], \quad H^{*}(\Delta ; \mathbb{k}):=\mathbb{k}[K] / \Theta,
$$

and, for short, $H_{T}^{*}(\Delta):=H_{T}^{*}(\Delta ; \mathbb{R})$ and $H^{*}(\Delta):=H^{*}(\Delta ; \mathbb{R})$.

\subsection{Evaluation on Fundamental Class}

Let $x=x_{i_{1}}^{j_{1}} \ldots \cdots x_{i_{k}}^{j_{k}}$ be a monomial whose index set $\left\{i_{1}, \ldots, i_{k}\right\}$ is not a simplex of $K$. Then $\iota_{I}(x)=0$ for any $I \in K^{\langle n\rangle}$, according to (4.1). Therefore $\pi_{!}^{\Delta}(x)=0$. Hence $\pi_{!}^{\Delta}$ vanishes on the Stanley-Reisner ideal $I_{S R}$ and descends to the map

$$
\pi_{!}^{\Delta}: \mathbb{R}[K] \rightarrow \mathbb{R}\left[V^{*}\right]
$$

Since $\pi_{!}^{\Delta}$ is a map of $\mathbb{R}\left[V^{*}\right]$-modules, we may apply $\otimes \mathbb{R}\left[V^{*}\right] \mathbb{R}$ to $\pi_{!}^{\Delta}$. This gives a linear map

$$
\int_{\Delta}: H^{2 n}(\Delta) \rightarrow \mathbb{R} \cong \mathbb{R}\left[V^{*}\right] / \mathbb{R}\left[V^{*}\right]^{+}
$$

Definition 4.5 Let $\Delta=(K, w, \lambda)$ be a complete simplicial multi-fan. The map $\int_{\Delta}: H^{2 n}(\Delta) \rightarrow \mathbb{R}$ is called "the evaluation on the fundamental class of $\Delta$ ".

We denote the composite map $\mathbb{R}\left[x_{1}, \ldots, x_{m}\right] \rightarrow H^{2 n}(\Delta) \rightarrow \mathbb{R}$ by $\int_{\Delta, \mathbb{R}[m]}$. 


\subsection{Chern Class of a Multi-Polytope}

Let $P$ be a multi-polytope based on a complete simplicial multi-fan $\Delta=(K, w, \lambda)$ of dimension $n$, and let $c_{1}, \ldots, c_{m} \in \mathbb{R}$ be the support parameters of $P$. The element

$$
c_{1}(P):=c_{1} x_{1}+\cdots+c_{m} x_{m} \in H^{2}(\Delta)
$$

is called the first Chern class of $P$.

\section{Proposition 4.6}

$$
\operatorname{Vol}(P)=\frac{1}{n !} \int_{\Delta} c_{1}(P)^{n}
$$

Proof If $\lambda$ and $w$ are integral, the statement is proved in Hattori and Masuda (2003, Lm. 8.6). The rational case follows from the integral case by the following arguments. (1) In the rational case we may choose a refined lattice such that $\lambda$ becomes integral with respect to this lattice (this would change the Euclidean measure on $V^{*}$, but this change affects both sides of (4.4) in the same way). (2) A rational weight $w$ may be turned into an integral weight by rescaling (both sides of (4.4) depend linearly on $w$, thus rescaling of $w$ preserves (4.4)). Real case follows by continuity, since both sides of (4.4) depend continuously on $\lambda$ and $w$.

It is easily seen that, for a given $\Delta$, the expression on the right hand side of (4.4) is a homogeneous polynomial of degree $n$ in the variables $c_{1}, \ldots, c_{m}$ :

$$
V_{\Delta}\left(c_{1}, \ldots, c_{m}\right)=\frac{1}{n !} \int_{\Delta}\left(c_{1} x_{1}+\cdots+c_{m} x_{m}\right)^{n} .
$$

Thus Proposition 4.6 implies Theorem 1.1.

\section{Basic Properties of Volume Polynomials}

\subsection{Partial Derivatives of Volume Polynomial}

We continue to assume that there is a fixed inner product in $V$ which makes the integral lattice in $V$ unnecessary. The inner product allows to identify $V$ and $V^{*}$ and to introduce a measure on each affine subspace of $V$ or $V^{*}$. Consider the space $\Lambda^{k} V$ of exterior forms on $V$. Given an inner product in $V$ we obtain an inner product on $\Lambda^{k} V$.

Suppose that every simplex $I \in K$ is oriented somehow. For a characteristic function $\lambda:[m] \rightarrow V$ on $K$ and $I=\left\{i_{1}, \ldots, i_{k}\right\} \in K$ let $\lambda(I)$ denote the skew form $\lambda\left(i_{1}\right) \wedge \cdots \wedge \lambda\left(i_{k}\right) \in \Lambda^{k} V$, where $\left(i_{1}, \ldots, i_{k}\right)$ is the positive order of vertices of $I$ (that is the order which defines the positive orientation of a simplex). Denote the norm of $\lambda(I)$ by $\operatorname{covol}(I)$ :

$$
\operatorname{covol}(I):=\|\lambda(I)\|=\left\|\lambda\left(i_{1}\right) \wedge \cdots \wedge \lambda\left(i_{k}\right)\right\| .
$$


Recall from Sect. 3 the notion of a face of a multi-polytope. If $P$ is a multi-polytope of dimension $n$ and $I \in K$ then $F_{I}$ is a multi-polytope of dimension $n-|I|$ sitting in the affine subspace $H_{I} \subset V^{*}$. There is a measure on $H_{I}$ determined by the inner product, hence we may define the volume of $F_{I}$. The following lemma shows that we can compute the volumes of faces from the volume polynomial.

Lemma 5.1 (cf. Timorin 1999, Thm. 2.4.3) Let $J \subset[m]$ and let $\partial_{J}$ denote the differential operator $\prod_{i \in J} \partial_{i}$. Consider the homogeneous polynomial $\partial_{J} V_{\Delta}$ of degree $n-|J|$. Then

1. Let $\theta_{u}$ denote the linear differential operator $\sum_{i=1}^{m}\langle u, \lambda(i)\rangle \partial_{i}$ for $u \in V^{*}$. Then $\theta_{u} V_{\Delta}=0$.

2. If $J \notin K$, then $\partial_{J} V_{\Delta}=0$;

3. If $J \in K$, then the value of the polynomial $\partial_{J} V_{\Delta}$ at a point $\left(\tilde{c}_{1}, \ldots, \tilde{c}_{m}\right) \in \mathbb{R}^{m}$ is equal to

$$
\frac{\operatorname{Vol} F_{J}}{\operatorname{covol}(J)}
$$

when $|J|<n$ and

$$
\frac{w(J)}{\operatorname{covol}(J)}=\frac{w(J)}{\left|\operatorname{det} \lambda_{J}\right|}
$$

when $|J|=n$. Here $\tilde{c}_{i}$ are the support parameters of a multi-polytope $P$ and $F_{J}$ are its faces.

Proof (1) We have

$$
\begin{aligned}
\theta_{u} V_{\Delta} & =\frac{1}{n !} \int\left(\sum_{i=1}^{m}\langle u, \lambda(i)\rangle \frac{\partial}{\partial c_{i}}\right)\left(c_{1} x_{1}+\cdots+c_{m} x_{m}\right)^{n} \\
& =\frac{1}{(n-1) !} \int\left(\sum_{i=1}^{m}\langle u, \lambda(i)\rangle x_{i}\right) \cdot\left(c_{1} x_{1}+\cdots+c_{m} x_{m}\right)^{n-1}=0,
\end{aligned}
$$

since $\sum_{i=1}^{m}\langle u, \lambda(i)\rangle x_{i}=0$ in $H^{*}(\Delta)$.

(2) The proof of second statement is completely similar to (1). We have

$$
\begin{aligned}
\partial_{J} V_{\Delta} & =\frac{1}{n !} \int\left(\prod_{i \in J} \frac{\partial}{\partial c_{i}}\right)\left(c_{1} x_{1}+\cdots+c_{m} x_{m}\right)^{n} \\
& =\frac{1}{(n-|J|) !} \int\left(\prod_{i \in J} x_{i}\right) \cdot\left(c_{1} x_{1}+\cdots+c_{m} x_{m}\right)^{n-|J|}=0,
\end{aligned}
$$

since $x_{J}=\prod_{i \in J} x_{i}=0$ in $H^{*}(\Delta)$ for $J \notin K$. 
(3) The second claim requires some technical work. At first, let $|J|=n$, i.e. $J \in K^{\langle n\rangle}$. We have

$$
\partial_{J} V_{\Delta}=\partial_{J} \frac{1}{n !} \int_{\Delta}\left(c_{1} x_{1}+\cdots+c_{m} x_{m}\right)^{n}=\int_{\Delta} x_{J}=\pi_{!}^{\Delta}\left(x_{J}\right),
$$

where $x_{J}=\prod_{i \in J} x_{i}$. By the definition of the index map (4.2) we have

$$
\pi_{!}^{\Delta}\left(x_{J}\right)=\sum_{I \in K^{\langle n\rangle}} \frac{w(I) \iota_{I}\left(x_{J}\right)}{\left|\operatorname{det} \lambda_{I}\right| \prod_{i \in I} \iota_{I}\left(x_{i}\right)} .
$$

If $I \neq J$, the corresponding summand vanishes, since $\iota_{I}\left(x_{j}\right)=0$ for $j \notin I$ by (4.1). The summand corresponding to $I=J$ contributes $\frac{w(J)}{\left|\operatorname{det} \lambda_{J}\right|}$ which proves the statement.

Let us prove the case $|J|<n$. Recall that the projected multi-fan $\Delta_{J}=$ $\left(\mathrm{lk}_{K} J, w_{J}, \lambda_{J}\right)$ is the multi-fan in the vector space $V_{J}=V /\langle\lambda(j) \mid j \in J\rangle$. There exists a "restriction" map

$$
\varphi_{J}: H^{*}(\Delta) \rightarrow H^{*}\left(\Delta_{J}\right)
$$

defined as follows:

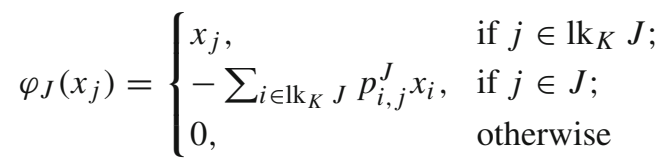

Here the constants $p_{i, j}^{J}$ for $j \in J$ and $i \in \mathrm{lk}_{K} J$ are defined by

$$
\operatorname{proj}_{J} \lambda(i)=\sum_{j \in J} p_{i, j}^{J} \lambda(j)
$$

where $\operatorname{proj}_{J} \lambda(i)$ is the orthogonal projection of the vector $\lambda(i)$ to the linear subspace spanned by $\lambda(j)(j \in J)$.

The homomorphism $\varphi_{J}$ is now defined on the level of polynomial algebras.

Claim 5.2 $\varphi_{J}$ is a well-defined ring homomorphism from $H^{*}(\Delta)=\mathbb{R}[K] / \Theta$ to $H^{*}\left(\Delta_{J}\right)=\mathbb{R}\left[\mathrm{lk}_{K} J\right] / \Theta_{J}$.

Proof The proof is a routine check. First let us prove that Stanley-Reisner relations in $\Delta$ are mapped to the Stanley-Reisner ideal of $\Delta_{J}$. Let $I$ be a non-simplex of $K$. The definition of $\varphi_{J}$ implies that $\varphi_{J}\left(x_{I}\right)=0$ unless $I \subset J \cup \operatorname{Vert}\left(\operatorname{lk}_{K} J\right)$. If $I \subset J \cup \operatorname{Vert}\left(\mathrm{lk}_{K} J\right)$, we have that $I \cap \operatorname{Vert}\left(\mathrm{lk}_{K} J\right)$ is a non-simplex of $1 \mathrm{k}_{K} J$ (otherwise we would have $I \in K$ contradicting the assumption). Then the element $\varphi_{J}\left(x_{I}\right)=$ $\varphi_{J}\left(\prod_{i \in I \cap \operatorname{Vert}\left(\mathrm{k}_{K} J\right)} x_{i}\right) \cdot \varphi_{J}\left(\prod_{i \in I \cap J} x_{i}\right)=\prod_{i \in I \cap \operatorname{Vert}\left(\mathrm{k}_{K} J\right)} x_{i} \cdot \varphi_{J}\left(\prod_{i \in I \cap J} x_{i}\right)$ lies in the Stanley-Reisner ideal of $\operatorname{lk}_{K} J$. 
Let us check that linear relations in $H^{*}(\Delta)$ are mapped into linear relations of $H^{*}\left(\Delta_{J}\right)$. A general linear relation in $H^{*}(\Delta)$ has the form $\sum_{i \in[m]}\langle u, \lambda(i)\rangle x_{i}$ for some $u \in V^{*}$. The map $\varphi_{J}$ sends it to the element

$$
\begin{aligned}
& \sum_{i \in \operatorname{Vert}\left(\mathrm{lk}_{K} J\right)}\left(\langle u, \lambda(i)\rangle-\sum_{j \in J} p_{i, j}^{J}\langle u, \lambda(j)\rangle\right) x_{i} \\
= & \sum_{i \in \operatorname{Vert}\left(\mathrm{k}_{K} J\right)}\left\langle u, \lambda(i)-\sum_{j \in J} p_{i, j}^{J} \lambda(j)\right\rangle x_{i}=\sum_{i \in \operatorname{Vert}\left(\mathrm{k}_{K} J\right)}\left\langle u, \lambda_{J}(i)\right\rangle x_{i} .
\end{aligned}
$$

(note that $\lambda(i)-\sum_{j \in J} p_{i, j}^{J} \lambda(j)=\lambda(i)-\operatorname{proj}_{J} \lambda(i)=\lambda_{J}(i)$ is the projection of $\lambda(i)$ to the plane orthogonal to $\left.\langle\lambda(j)\rangle_{j \in J}\right)$. The last expression is zero in $H^{*}\left(\Delta_{J}\right)$.

Next we show that restriction homomorphism is compatible with the first Chern classes of the multi-polytopes.

Claim $5.3 \varphi_{J}$ sends $c_{1}(P)$ to $c_{1}\left(F_{J}\right)$.

Proof Recall that $H_{J}$ denotes the ambient space of the face $F_{J}$ of the multi-polytope $P$. The supporting hyperplanes of $F_{J}$ are given by intersections $H_{J} \cap H_{i}$, where $H_{i}$ is the supporting hyperplane of $P$ for $i \in \mathrm{lk}_{K} J$.

Let us denote by $U_{J}$ the subspace spanned by $\lambda(j)$ 's $(j \in J)$ so that $V_{J}=V / U_{J}$. By the definition (see Sect. 2.4), $\lambda_{J}(i)$ is the projection image of $\lambda(i)$ on $V_{J}$ if $i$ is the vertex of $\mathrm{lk}_{K} J$. As in the proof of previous claim we identify the quotient space $V_{J}=V / U_{J}$ with the orthogonal complement $U_{J}^{\perp}$ of $U_{J}$. The projected vector $\lambda_{J}(i)$ can be considered as the element in $V$ and we have

$$
\lambda(i)=\lambda_{J}(i)+\operatorname{proj}_{J} \lambda(i)
$$

with respect to the orthogonal decomposition $V=U_{J}^{\perp} \oplus U_{J}$.

The affine hyperplane $H_{i}$ is given by $\left\{u \in V^{*} \mid\langle u, \lambda(i)\rangle=c_{i}\right\}$. The affine plane $H_{J}$ is given by $\left\{u \in V^{*} \mid\langle u, \lambda(j)\rangle=c_{j}\right.$, for all $\left.j \in J\right\}$. By using (5.3) and (5.4) we may write the intersection $H_{i} \cap H_{J}$ as

$$
\begin{aligned}
\{u & \left.\in H_{J} \mid\left\langle u, \lambda_{J}(i)+\operatorname{proj}_{J} \lambda(i)\right\rangle=c_{i}\right\} \\
& =\left\{u \in H_{J} \mid\left\langle u, \lambda_{J}(i)+\sum_{j \in J} p_{i, j}^{J} \lambda(j)\right\rangle=c_{i}\right\} \\
& =\left\{u \in H_{J} \mid\left\langle u, \lambda_{J}(i)\right\rangle=c_{i}-\sum_{j \in J} p_{i, j}^{J} c_{j}\right\} .
\end{aligned}
$$

Therefore the $i$-th support parameter of $F_{J}$ is $c_{i}-\sum_{j \in J} p_{i, j}^{J} c_{j}$ for $i \in \mathrm{lk}_{K} J$. Now it remains to note that the coefficient of $x_{i}$ in the projected class $\varphi_{J}\left(c_{1}(P)\right)$ is exactly $c_{i}-\sum_{j \in J} p_{i, j}^{J} c_{j}$. Thus $\varphi_{J}\left(c_{1}(P)\right)=c_{1}\left(F_{J}\right)$. 
Now we prove the following

\section{Claim 5.4}

$$
\int_{\Delta} y \prod_{j \in J} x_{j}=\frac{1}{\operatorname{covol}(J)} \int_{\Delta_{J}} \varphi_{J}(y) \text { for any } y \in H^{*}(\Delta)
$$

Proof Let us denote by $\operatorname{Vol} S$ the volume of the parallelepiped formed by a set of vectors $S$. Then $\operatorname{covol}(J)=\operatorname{Vol}\{\lambda(i)\}_{i \in J}$ and the index map can be written as

$$
\pi_{!}^{\Delta}(x)=\sum_{I \in K^{\langle n\rangle}} \frac{w(I) \iota_{I}(x)}{\operatorname{Vol}\{\lambda(i)\}_{i \in I} \prod_{i \in I} \iota_{I}\left(x_{i}\right)} .
$$

Let $\tilde{I} \in \mathrm{lk}_{K} J$ and, therefore, $\tilde{I} \sqcup J \in K$. Then

$$
\operatorname{Vol}\{\lambda(i)\}_{i \in \tilde{I} \sqcup J}=\operatorname{Vol}\{\lambda(i)\}_{i \in J} \cdot \operatorname{Vol}\left\{\lambda_{J}(i)\right\}_{i \in \tilde{I}}=\operatorname{covol}(J) \cdot \operatorname{Vol}\{\lambda J(i)\}_{i \in \tilde{I}}
$$

This together with (5.5) implies the lemma.

Applying Claim 5.4 to $y=c_{1}(P)^{n-|J|}$ and using Claim 5.3, we obtain

$$
\partial_{J} V_{\Delta}=\frac{1}{(n-|J|) !} \int_{\Delta} c_{1}(P)^{n-|J|} \prod_{j \in J} x_{j}=\frac{1}{\operatorname{covol}(J)} \frac{1}{(n-|J|) !} \int_{\Delta_{J}} c_{1}\left(F_{J}\right)^{n-|J|} .
$$

Expression at the right evaluates to $\frac{\operatorname{Vol}\left(F_{J}\right)}{\operatorname{covol}(J)}$ which finishes the proof of Lemma 5.1.

Corollary 5.5 Let $\Delta=\left(w_{c h}, \lambda\right)$ be a complete multi-fan. Then $V_{\Delta}=0$ implies $w_{c h}=0$.

Proof If $V_{\Delta}=0$, then $\partial_{J} V_{\Delta}=0$ for any $J \in K^{\langle n\rangle}$. This implies $w_{c h}=0$.

Remark 5.6 Of course, according to Proposition 7.2 the polynomial $V_{\Delta}$ is non-zero if and only if the map $\int_{\Delta}$ is non-zero. The fact that $\int_{\Delta}$ is non-zero for every nonzero $w_{c h}$ is proved by applying this map to all monomials $x_{I}, I \in K^{\langle n\rangle}$ (recall that these monomials span $H^{2 n}(\Delta)$ ). This procedure is essentially the same as applying differential operators $\partial_{I}$ to $V_{\Delta}$.

Corollary 5.7 Let $\partial_{P}$ denote the linear differential operator $\sum_{i \in[m]} \tilde{c}_{i} \partial_{i}$ where $\tilde{c}_{i}$ are the support parameters of a multi-polytope $P$. Then

$$
\begin{gathered}
\frac{1}{n !} \partial_{P}^{n} V_{\Delta}=\operatorname{Vol}(P) \\
\frac{1}{(n-|I|) !} \partial_{P}^{n-|I|} \partial_{I} V_{\Delta}=\frac{\operatorname{Vol}\left(F_{I}\right)}{\operatorname{covol}(I)}
\end{gathered}
$$


Proof Both formulas follow from Lemma 5.1 and a simple observation: if $\Psi \in$ $\mathbb{R}\left[c_{1}, \ldots, c_{m}\right]_{k}$ is a homogeneous polynomial of degree $k$, then

$$
\left(\sum_{i \in[m]} \tilde{c}_{i} \partial_{i}\right)^{k} \Psi=k ! \Psi\left(\tilde{c}_{1}, \ldots, \tilde{c}_{m}\right)
$$

(evaluation at a point coincides with the result of differentiation up to $k$ !).

\subsection{Recovering Multi-Fans from Volume Polynomials}

When we associate a volume polynomial to a complete simplicial multi-fan, the numbering of the one-dimensional cones by $[\mathrm{m}]$ is incorporated in the data of the multi-fan. We call a multi-fan with the numbering a based multi-fan. Two based multi-fans $\Delta$ and $\Delta^{\prime}$ are said to be equivalent if there is an automorphism of $V$ which induces an isomorphism between $\Delta$ and $\Delta^{\prime}$ preserving the numbering. In the presence of a lattice $N \subset V$ there should be an automorphism of the lattice with this property. Equivalent complete simplicial based multi-fans have the same volume polynomial. We will see that the converse holds for complete simplicial based multi-fans $\Delta$ whose underlying simplicial complexes are oriented strongly connected pseudo-manifolds. Strong connectedness of $K$ means that for any two maximal simplices $I, I^{\prime} \in K^{\langle n\rangle}$ there exists a sequence of maximal simplices $I=I_{0}, I_{1}, \ldots, I_{k}=I^{\prime}$ such that $\left|I_{s} \cap I_{s+1}\right|=n-1$ for $0 \leqslant s \leqslant k-1$.

We assume that the volume polynomial $V_{\Delta}$ associated to $\Delta$ is non-zero. Then the class [ $\Delta$ ] is non-zero. Since $K$ is assumed to be a pseudo-manifold, $w(I) \neq 0$ for any $I \in K^{\langle n\rangle}$. Then Lemma 5.1 shows that $V_{\Delta}$ recovers $K$.

Remember that

$$
\sum_{i=1}^{m}\langle u, \lambda(i)\rangle x_{i}=0 \text { in } H^{*}(\Delta) \text { for any } u \in V^{*}
$$

Let $J \in K,|J|=n-1$. Since $K$ is assumed to be a pseudo-manifold, there are exactly two elements $i_{1}$ and $i_{2}$ in $[m]$ such that $J \cup\left\{i_{1}\right\}$ and $J \cup\left\{i_{2}\right\}$ are in $K^{\langle n\rangle}$. Multiplying $x_{J}=\prod_{i \in J} x_{i}$ to the both sides in (5.6), we obtain

$$
\sum_{j \in J}\langle u, \lambda(j)\rangle x_{j} x_{J}+\left\langle u, \lambda\left(i_{1}\right)\right\rangle x_{i_{1}} x_{J}+\left\langle u, \lambda\left(i_{2}\right)\right\rangle x_{i_{2}} x_{J}=0 \text { for all } u \in V^{*}
$$

Applying $\int_{\Delta}$ to the above identity, we have

$$
\left\langle u, \sum_{j \in J}\left(\int_{\Delta} x_{j} x_{J}\right) \lambda(j)+\left(\int_{\Delta} x_{i_{1}} x_{J}\right) \lambda\left(i_{1}\right)+\left(\int_{\Delta} x_{i_{2}} x_{J}\right) \lambda\left(i_{2}\right)\right\rangle=0 .
$$


Since this holds for all $u \in V^{*}$, one can conclude

$$
\sum_{j \in J}\left(\int_{\Delta} x_{j} x_{J}\right) \lambda(j)+\left(\int_{\Delta} x_{i_{1}} x_{J}\right) \lambda\left(i_{1}\right)+\left(\int_{\Delta} x_{i_{2}} x_{J}\right) \lambda\left(i_{2}\right)=0 .
$$

Note that the numbers $\int_{\Delta} x_{i_{1}} x_{J}$ and $\int_{\Delta} x_{i_{2}} x_{J}$ are non-zero. Identity (5.7) shows that once basis vectors $\{\lambda(i)\}_{i \in I}$ for some $I \in K^{\langle n\rangle}$ are determined, then the other vectors $\lambda(k)$ 's will be determined by the intersection numbers $\int_{\Delta} x_{\mathcal{I}}$ where $\mathcal{I}$ consists of elements in $[m]$ with $|\mathcal{I}|=n$ (an element in $\mathcal{I}$ may appear more than once). On the other hand, since

$$
V_{\Delta}=\frac{1}{n !} \int_{\Delta}\left(c_{1} x_{1}+\cdots+c_{m} x_{m}\right)^{n}
$$

the coefficient of $c^{\mathcal{I}}$ agrees with $\int_{\Delta} x_{\mathcal{I}}$ up to some non-zero constant independent of $\Delta$. These show that $V_{\Delta}$ determines $\Delta$ up to equivalence.

Proposition 5.8 Two complete simplicial toric varieties are isomorphic if and only if their volume polynomials agree up to permutations of variables. Here it is assumed that all $\lambda(i)$ 's are the primitive generators of the rays.

Proof This follows from the above observation and the fact that two toric varieties are isomorphic if and only if their fans are isomorphic (Berchtold 2003). ${ }^{1}$

\section{A Formula for the Volume Polynomial}

We say that the set $S$ of $n+1$ vectors in $V \cong \mathbb{R}^{n}$ is in general position, if any $n$ of them are linearly independent. Any such set determines a multi-fan whose underlying simplicial complex is a boundary of a simplex $K=\partial \triangle_{[n+1]}$. The weights of all maximal simplices are the same up to sign due to closedness condition $d w_{c h}=$ 0 . Thus without loss of generality we may assume that every weight is 1 or -1 depending on the orientation. We call such multi-fan an elementary multi-fan and denote it $\Delta^{e l}(S)$.

Lemma 6.1 Let $\Delta$ be an elementary multi-fan determined by the vectors $\lambda(1), \ldots$, $\lambda(n+1) \in V$. Let $0 \neq\left(\alpha_{1}, \ldots, \alpha_{n+1}\right) \in \mathbb{R}^{n+1}$ be a nonzero linear relation on these vectors, i.e. $\sum_{i=1}^{n+1} \alpha_{i} \lambda(i)=0$. Then

$$
V_{\Delta}\left(c_{1}, \ldots, c_{n+1}\right)=\text { const } \cdot\left(\alpha_{1} c_{1}+\cdots+\alpha_{n+1} c_{n+1}\right)^{n} .
$$

for some constant const.

We postpone the proof to Sect. 8.3.

\footnotetext{
1 We are grateful to Ivan Arzhantsev from whom we learned this fact.
} 
Remark 6.2 It is not difficult to compute the constant: just apply the differential operator $\partial_{J}$ for $J \subset[n+1],|J|=n$ to both sides of (6.1) and use Lemma 5.1. However, we do not need this constant at the moment and ignore it to simplify the exposition.

Theorem 6.3 Let $\Delta=\left(w_{c h}, \lambda\right)$ be a complete multi-fan. Let $v \in V$ be a generic vector. Then

$$
V_{\Delta}\left(c_{1}, \ldots, c_{m}\right)=\frac{1}{n !} \sum_{I=\left\{i_{1}, \ldots, i_{n}\right\} \in K} \frac{w(I)}{\left|\operatorname{det} \lambda_{I}\right| \prod_{j=1}^{n} \alpha_{I, j}}\left(\alpha_{I, 1} c_{i_{1}}+\cdots+\alpha_{I, n} c_{i_{n}}\right)^{n},
$$

where $\alpha_{I, 1}, \ldots, \alpha_{I, n}$ are the coordinates of $v$ in the basis $\left(\lambda\left(i_{1}\right), \ldots, \lambda\left(i_{n}\right)\right)$, and $w(I)$ is the weight.

Proof We derive a more general family of formulas, and (6.2) will be a particular case. Let $\left[\mathrm{m}^{\prime}\right]$ be a set containing $[\mathrm{m}]$ and let

$$
z_{c h}=\sum_{J \in\left(\begin{array}{l}
{\left[m^{\prime}\right]} \\
n+1
\end{array}\right)} z(J) J \in C_{n}\left(\triangle_{\left[m^{\prime}\right]} ; \mathbb{R}\right)
$$

be a simplicial chain such that $d z_{c h}=w_{c h}$ (it exists since $w_{c h}$, considered as an element in $C_{n}\left(\triangle_{\left[m^{\prime}\right]} ; \mathbb{R}\right)$, is closed hence exact). Consider any function $\eta:\left[\mathrm{m}^{\prime}\right] \rightarrow V$ which extends $\lambda:[m] \rightarrow V$ and satisfies the condition: for any $J=\left\{j_{1}, \ldots, j_{n+1}\right\}$ with $z(J) \neq 0$ the vectors $\eta\left(j_{1}\right), \ldots, \eta\left(j_{n+1}\right)$ are in general position. Thus for any such $J$ we can construct an elementary multi-fan $\Delta^{e l}(\eta(J))$.

In the group of multi-fans we have a relation $\Delta=\sum_{J \in\left(\begin{array}{c}{\left[m^{\prime}\right]} \\ n+1\end{array}\right)} z(J) \Delta^{e l}(\eta(J))$, if $\Delta$ is considered as a multi-fan on $\left[\mathrm{m}^{\prime}\right]$ (recall that multi-fans with the same characteristic function can be added to each other and multiplied by integers by performing this operations on their weights, and therefore such multi-fans form an abelian group). Volume polynomial is additive, hence we get

$$
V_{\Delta}=\sum_{J \in\left(\begin{array}{c}
{\left[m^{\prime}\right]} \\
n+1
\end{array}\right)} z(J) V_{\Delta^{e l}(\eta(J))}
$$

Therefore, any simplicial chain whose boundary is $w_{c h}$ gives a formula for the volume polynomial. Now let us consider the particular case, namely, the cone over $w_{c h}$. Let $\left[m^{\prime}\right]=[m] \sqcup\{r\}$ and set $\eta(r)=v$, for a generic vector $v \in V$. So the phrase " $v$ is a generic vector" means that the set $\lambda(I) \sqcup\{v\}$ is in general position for any $I \subset[m]$ such that $|I|=n$ and $w(I) \neq 0$. The function $z$ on the cone is defined in an obvious way: $z(I \sqcup\{r\}):=w(I)$.

Relation (6.3) and Lemma 6.1 imply

$$
V_{\Delta}=\sum_{I=\left\{i_{1}, \ldots, i_{n}\right\} \subset[m]} \text { const } \cdot w(I) \cdot\left(\alpha_{I, 1} c_{i_{1}}+\cdots+\alpha_{I, n} c_{i_{n}}+\beta_{I} c_{r}\right)^{n}
$$


The tuple $\left(\alpha_{I, 1}, \ldots, \alpha_{I, n}, \beta_{I}\right)$ is a linear relation on the vectors $\lambda\left(i_{1}\right), \ldots, \lambda\left(i_{n}\right), v$. Therefore we may assume that $\beta_{I}=-1$ and $\left(\alpha_{I, 1}, \ldots, \alpha_{I, n}\right)$ are the coordinates of $v$ in the basis $\lambda\left(i_{1}\right), \ldots, \lambda\left(i_{n}\right)$.

Left hand side of (6.4) does not depend on $c_{r}$ (it is a redundant support parameter), therefore we may put $c_{r}=0$ :

$$
V_{\Delta}=\sum_{I=\left\{i_{1}, \ldots, i_{n}\right\} \subset[m]} A_{I} \cdot w(I) \cdot\left(\alpha_{I, 1} c_{i_{1}}+\cdots+\alpha_{I, n} c_{i_{n}}\right)^{n} .
$$

To compute the constants $A_{I}$ take any $J=\left\{j_{1}, \ldots, j_{n}\right\} \in K$ and apply the differential operator $\partial_{J}=\frac{\partial}{\partial c_{j_{1}}} \cdots \cdots \frac{\partial}{\partial c_{j_{n}}}$ to the identity (6.5). On the left we have $\partial_{J} V_{\Delta}=\frac{w(J)}{\left|\operatorname{det} \lambda_{J}\right|}$, according to Lemma 5.1. On the right side all summands with $I \neq J$ vanish, and the one with $I=J$ contributes $n ! \cdot A_{J} \cdot w(J) \prod_{i} \alpha_{J, i}$. Thus $A_{J}=\frac{1}{n !} \frac{1}{\left|\operatorname{det} \lambda_{J}\right| \cdot \prod_{i} \alpha_{J, i}}$ and the statement follows.

Remark 6.4 Note that the formula (6.2) can be applied to compute the volume of a simple convex polytope in the case when the polytope is described as the intersection of half-spaces with the given equations. In this case the formula is known as Lawrence's formula (Lawrence 1991). It has found applications in explicit volumes' calculations.

Example 6.5 Consider the standard fan $\Delta$ of $\mathbb{C} P^{2}$, generated by the vectors $\lambda(1)=$ $(1,0), \lambda(2)=(0,1), \lambda(3)=(-1,-1)$. Take the generic vector $v=(1,2)$. We have

$$
v=\lambda(1)+2 \lambda(2)=\lambda(2)-\lambda(3)=-2 \lambda(3)-\lambda(1) .
$$

Theorem 6.3 implies

$$
V_{\Delta}=\frac{1}{2}\left(\frac{1}{2}\left(c_{1}+2 c_{2}\right)^{2}-\left(c_{2}-c_{3}\right)^{2}+\frac{1}{2}\left(-c_{1}-2 c_{3}\right)^{2}\right) \text {. }
$$

This expression equals $\frac{1}{2}\left(c_{1}+c_{2}+c_{3}\right)^{2}$. The same expression is given by Lemma 6.1.

Example 6.6 Consider the normal fan of the standard $n$-cube. The underlying simplicial complex is isomorphic to the boundary of cross-polytope. Let $\{1, \ldots, n,-1, \ldots$, $-n\}$ be its set of vertices, so the maximal simplices have the form $\{ \pm 1, \ldots, \pm n\}$. We have $\lambda( \pm i)= \pm e_{i}$. Take the generic vector $v=e_{1}+\cdots+e_{n}$. Then Theorem 6.3 implies

$$
V_{\Delta}=\frac{1}{n !} \sum_{\left(\epsilon_{1}, \ldots, \epsilon_{n}\right) \in\{+,-\}^{n}} \frac{1}{\prod_{i=1}^{n} \epsilon_{i}}\left(\epsilon_{1} c_{\epsilon_{1} 1}+\cdots+\epsilon_{n} c_{\epsilon_{n} n}\right)^{n}
$$

On the other hand, we have $V_{\Delta}=\prod_{i}\left(c_{i}+c_{-i}\right)$ by geometrical reasons. Indeed, the polytope dual to $\Delta$ is the brick with sides $\left\{c_{i}+c_{-i}\right\}_{i \in[n]}$. By setting $c_{-i}=0$ for each $i$ we get the identity 


$$
\prod_{i=1}^{n} c_{i}=\frac{1}{n !} \sum_{I \subseteq[n]}(-1)^{n-|I|} c_{I}^{n}
$$

where $c_{I}=\sum_{i \in I} c_{i}$. This identity is well known as the discrete polarization identity.

Remark 6.7 The proof of Theorem 6.3 implies the following consideration. Take two simplicial $n$-chains $z_{c h, 1}, z_{c h, 2} \in C_{n}\left(\triangle_{[m]} ; \mathbb{R}\right)$ endowed with functions $\eta_{1}, \eta_{2}:[m] \rightarrow$ $\mathbb{R}^{n}$ such that $\eta_{\epsilon}(J)$ is in general position for any simplex $J$ of the chain $z_{c h, \epsilon}, \epsilon=$ 1,2. Assume that $d z_{c h, 1}=d z_{c h, 2}$ and the functions $\eta_{1}, \eta_{2}$ agree on the vertices of the boundary. Then the volume polynomial of the multi-fan $\Delta=\left(d z_{c h, 1}, \eta_{1}\right)=$ $\left(d z_{c h, 2}, \eta_{2}\right)$ can be expressed by two formulas:

$$
\begin{aligned}
& \sum_{J=\left(j_{1}, \ldots, j_{n+1}\right) \subset\left[m^{\prime}\right]} \operatorname{const} \cdot z_{1}(J)\left(\alpha_{J, 1} c_{j_{1}}+\cdots+\alpha_{J, n+1} c_{j_{n+1}}\right)^{n}=V_{\Delta} \\
= & \sum_{J=\left(j_{1}, \ldots, j_{n+1}\right) \subset\left[m^{\prime}\right]} \operatorname{const} \cdot z_{2}(J)\left(\alpha_{J, 1} c_{j_{1}}+\cdots+\alpha_{J, n+1} c_{j_{n+1}}\right)^{n} .
\end{aligned}
$$

We may take a difference of the left and right parts and summarize as follows. Let us take any closed simplicial $n$-chain $z_{c h}, d z_{c h}=0$, on the vertex set $\left[\mathrm{m}^{\prime}\right]$, and endow it with a function $\eta:\left[m^{\prime}\right] \rightarrow \mathbb{R}^{n}$ which is in general position on any simplex $J$ of the chain. Then we get an identity

$$
\sum_{J=\left(j_{1}, \ldots, j_{n+1}\right) \subset\left[m^{\prime}\right]} \operatorname{const} \cdot z(J)\left(\alpha_{J, 1} c_{j_{1}}+\cdots+\alpha_{J, n+1} c_{j_{n+1}}\right)^{n}=0
$$

(the constants may be computed by the same method as we used previously). This seems to be a quite general way to construct algebraical identities from geometrical data.

This idea can be illustrated by a simple identity obtained in Example 6.5:

$$
\frac{1}{2}\left(c_{1}+2 c_{2}-c_{4}\right)^{2}-\left(c_{2}-c_{3}-c_{4}\right)^{2}+\frac{1}{2}\left(-c_{1}-2 c_{3}-c_{4}\right)^{2}=\left(c_{1}+c_{2}+c_{3}\right)^{2} .
$$

This identity is induced by the schematic picture shown on Fig. 3. Note that the last step in the proof of Theorem 6.3 was to specialize $c_{4}=0$, but even without this specialization the identity holds true.
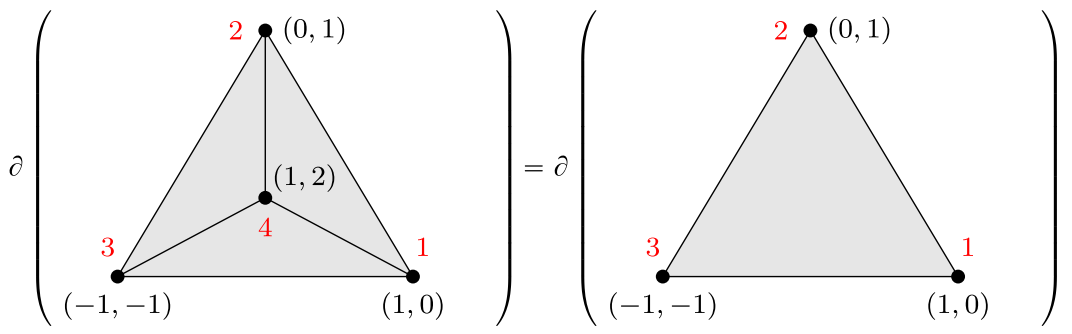

Fig. 3 Two simplicial chains with vector functions having the same boundary 


\section{Poincare Duality Algebra of a Multi-Fan}

\subsection{Poincare Duality Algebras}

Definition 7.1 Let $\mathbb{k}$ be a field. Let $\mathcal{A}^{*}=\bigoplus_{j=0}^{n} \mathcal{A}^{2 j}$ be a finite-dimensional graded commutative $\mathbb{k}$-algebra such that

- there exists an isomorphism $\int_{\mathcal{A}}: \mathcal{A}^{2 n} \rightarrow \mathbb{k}$;

- the pairing $\mathcal{A}^{2 p} \otimes \mathcal{A}^{2 n-2 p} \rightarrow \mathbb{k}, a \otimes b \mapsto \int_{\mathcal{A}}(a \cdot b)$ is non-degenerate.

Then $\mathcal{A}$ is called a Poincare duality algebra of formal dimension $2 n$.

Let $\partial_{i}=\frac{\partial}{\partial c_{i}}, i \in[m]$ be the differential operator acting on the ring of polynomials $\mathbb{R}\left[c_{1}, \ldots, c_{m}\right]$ in a standard way. For a subset $I \subset[m]$ let $\partial_{I}$ denote the product $\prod_{i \in I} \partial_{i}$

Consider the algebra of differential operators with constant coefficients $\mathcal{D}:=$ $\mathbb{R}\left[\partial_{1}, \ldots, \partial_{m}\right]$. It will be convenient to double the degree, so we assume deg $\partial_{i}=2$, $i \in[m]$ (while still assuming that $\operatorname{deg} c_{i}=1$ ). For any non-zero homogeneous polynomial $\Psi \in \mathbb{R}\left[c_{1}, \ldots, c_{m}\right]$ of degree $n$ we may consider the following ideal in $\mathcal{D}$ :

$$
\text { Ann } \Psi:=\{D \in \mathcal{D} \mid D \Psi=0\}
$$

It is not difficult to check that the quotient $\mathcal{D} / \operatorname{Ann} \Psi$ is a Poincare duality algebra of formal dimension $2 n$ (see Timorin 1999, Prop. 2.5.1), where the "integration map" assigns the number $D \Psi \in \mathbb{R}$ to any differential operator of rank $n$ (i.e. of formal degree $2 n$ in our setting).

It happens that every Poincare duality algebra generated by degree two can be obtained by this construction as the following proposition shows.

Proposition 7.2 Suppose char $\mathbb{k}=0$ and let $\mathbb{k}[m]=\mathbb{k}\left[x_{1}, \ldots, x_{m}\right]$ be a polynomial ring, where deg $x_{i}=2$. Then the following three sets of objects are naturally equivalent:

1. Poincare duality algebras $\mathcal{A}^{*}$ of formal dimension $2 n$ which are the quotients of the polynomial ring $\mathbb{k}[m]$;

2. Non-zero homogeneous polynomials $\Psi \in \mathbb{k}\left[c_{1}, \ldots, c_{m}\right]$ of degree $n$ (where $\left.\operatorname{deg} c_{i}=1\right)$ up to multiplication by a non-zero constant;

3. Non-zero linear maps $\int: \mathbb{k}[m]_{2 n} \rightarrow \mathbb{k}$ up to multiplication by a non-zero constant.

Proof We give a very brief sketch of the proof. For details the reader is referred to the monograph (Meyer and Smith 2005) which, among other things, describes the case char $\mathbb{k} \neq 0$ (for general fields instead of a polynomial $\Psi$ one should take an element of divided power algebra). Also we would like to mention that an equivalence of (1) and (2) is a manifestation of the well-known phenomenon called Macaulay duality (or its extended version, Matlis duality). 
(1) $\Rightarrow(3)$. Let $\mathcal{A}^{*} \cong \mathbb{k}[m] / \mathcal{I}$ be a Poincare duality quotient of the ring of polynomials. Then we have a linear isomorphism $\int_{\mathcal{A}}: \mathcal{A}^{2 n} \rightarrow \mathbb{k}$. The composite

$$
\mathbb{k}[m]_{2 n} \rightarrow \mathcal{A}^{2 n} \rightarrow \mathbb{k}
$$

is the required linear map.

$(3) \Rightarrow(1)$. Given a linear map $\int: \mathbb{k}[m]_{2 n} \rightarrow \mathbb{k}$ we may define a pairing $\mathbb{k}[m]_{2 p} \otimes$ $\mathbb{k}[m]_{2 n-2 p} \rightarrow \mathbb{k}$ by $a \otimes b \mapsto \int a \cdot b$. This pairing is degenerate and we define its kernel:

$$
W^{*}=\bigoplus_{p} W^{2 p}, \quad W^{2 p}=\left\{x \in \mathbb{k}[m]_{2 p} \mid \int x \cdot \mathbb{k}[m]_{2 n-2 p}=0\right\} .
$$

It is easy to check that $W^{*} \subset \mathbb{k}[m]$ is an ideal and $\mathbb{k}[m] / W^{*}$ is a Poincare duality algebra.

(3) $\Rightarrow(2)$. We construct a polynomial $\Psi$ in $c_{1}, \ldots, c_{m}$ by

$$
\Psi:=\frac{1}{n !} \int\left(c_{1} x_{1}+\cdots+c_{m} x_{m}\right)^{n}
$$

This polynomial is non-zero. Indeed, $\mathbb{k}[\mathrm{m}]_{2 n}$ is additively generated by the monomials of degree $n$ in the variables $x_{i}$. Each monomial can be expressed as a linear combination of expressions of the form $\left(c_{1} x_{1}+\cdots+c_{m} x_{m}\right)^{n}$ for some constants $c_{i}$ as follows from the polarization identity (see (6.6) in Example 6.6). Thus expressions of the form $\left(c_{1} x_{1}+\cdots+c_{m} x_{m}\right)^{n}$ linearly span $\mathbb{k}[m]_{2 n}$ and therefore, since $\int$ is non-zero, the polynomial $\Psi$ is not a constant zero as well.

$(2) \Rightarrow(1)$. Given a homogeneous polynomial $\Psi$ in the variables $c_{1}, \ldots, c_{m}$, we may construct a Poincare duality quotient $\mathbb{k}\left[\partial_{1}, \ldots, \partial_{m}\right] /$ Ann $\Psi$, where the action of $\partial_{i}=\frac{\partial}{\partial c_{i}}$ on polynomials is defined formally in the usual way.

The consistency of all these constructions is a routine check.

The same arguments can be used to prove that there is a one-to-one correspondence between Poincare duality quotients of formal dimension $2 n$ of an algebra $\mathcal{B}^{*}$ and the non-zero linear functionals on the linear space $\mathcal{B}^{2 n}$. For this correspondence we do not need the assumptions that $\mathcal{B}$ is generated by degree 2 and char $\mathbb{k}=0$. This motivates the following definition.

Definition 7.3 Let $\mathcal{B}^{*}=\bigoplus_{j} \mathcal{B}^{2 j}$ be a graded commutative $\mathbb{k}$-algebra and suppose that for some $n>0$ a non-zero linear map $\int: \mathcal{B}^{2 n} \rightarrow \mathbb{k}$ is given. The corresponding Poincare duality quotient of $\mathcal{B}^{*}$, i.e. the algebra

$$
\mathcal{B}^{*} / W^{*}, \quad W^{2 p}=\left\{b \in \mathcal{B}^{2 p} \mid \int b \cdot \mathcal{B}^{2 n-2 p}=0\right\} .
$$

is denoted by $\operatorname{PD}\left(\mathcal{B}^{*}, \int\right)$ and called Poincare dualization of $\mathcal{B}^{*}$ (w.r.t. $\int$ ). 
Lemma 7.4 Consider two algebras $\mathcal{B}_{1}^{*}, \mathcal{B}_{2}^{*}$ with the given non-zero linear maps $\int_{1}: \mathcal{B}_{1}^{2 n} \rightarrow \mathbb{k}, \int_{2}: \mathcal{B}_{2}^{2 n} \rightarrow \mathbb{k}$. Let $\varphi: \mathcal{B}_{1}^{*} \rightarrow \mathcal{B}_{2}^{*}$ be an epimorphism of algebras consistent with the integration maps: $\left.\int_{2} \circ \varphi\right|_{\mathcal{B}_{1}^{2 n}}=\int_{1}$. Then $\varphi$ induces an isomorphism $\operatorname{PD}\left(\mathcal{B}_{1}^{*}, \int_{1}\right) \cong \operatorname{PD}\left(\mathcal{B}_{2}^{*}, \int_{2}\right)$.

Proof From the surjectivity of $\varphi$ it easily follows that the kernel $W_{1}^{*}$ of the intersection pairing in the first algebra maps to the kernel $W_{2}^{*}$ of the second algebra. Thus the homomorphism $\widetilde{\varphi}: \operatorname{PD}\left(\mathcal{B}_{1}^{*}, \int_{1}\right) \rightarrow \operatorname{PD}\left(\mathcal{B}_{2}^{*}, \int_{2}\right)$ is well defined. Obviously it is surjective. Let us prove that $\widetilde{\varphi}$ is injective. The map $\widetilde{\varphi}$ is an isomorphism in degree $2 n$. Suppose that $\widetilde{\varphi}(a)=0$ for some $0 \neq a \in \operatorname{PD}\left(\mathcal{B}_{1}^{*}, \int_{1}\right)_{2 p}$. By the definition of Poincare duality algebra, there exists $b \in \operatorname{PD}\left(\mathcal{B}_{1}^{*}, \int_{1}\right)_{2 n-2 p}$ such that $a b \neq 0$. But then we have $\widetilde{\varphi}(a b)=\widetilde{\varphi}(a) \widetilde{\varphi}(b)=0$ which gives a contradiction.

In the following let $\mathcal{A}^{*}(\Psi)=\mathcal{D} /$ Ann $\Psi$ denote the Poincare duality algebra corresponding to the homogeneous polynomial $\Psi$ of degree $n$.

\subsection{Algebras Associated with Multi-Fans}

The linear maps $\int_{\Delta}: H^{2 n}(\Delta) \rightarrow \mathbb{R}$ and $\int_{\Delta, \mathbb{R}[m]}: \mathbb{R}\left[x_{1}, \ldots, x_{m}\right]_{2 n} \rightarrow \mathbb{R}$ are consistent with the natural projection $\mathbb{R}\left[x_{1}, \ldots, x_{m}\right] \rightarrow H^{*}(\Delta)$. Thus Lemma 7.4 implies an isomorphism

$$
\operatorname{PD}\left(H^{*}(\Delta), \int_{\Delta}\right) \cong \operatorname{PD}\left(\mathbb{R}[m], \int_{\Delta, \mathbb{R}[m]}\right)
$$

According to the constructions mentioned in the proof of Proposition 7.2, this Poincare duality algebra is also isomorphic to $\mathcal{A}^{*}\left(V_{\Delta}\right)=\mathcal{D} /$ Ann $V_{\Delta}$, where $V_{\Delta}$ is the volume polynomial.

Definition 7.5 Let $\Delta$ be a complete simplicial multi-fan of dimension $n$ with $m$ rays. Then the algebra

$$
\mathcal{A}^{*}(\Delta):=\mathcal{D} / \text { Ann } V_{\Delta} \cong \operatorname{PD}\left(H^{*}(\Delta), \int_{\Delta}\right) \cong \operatorname{PD}\left(\mathbb{R}[m], \int_{\Delta, \mathbb{R}[m]}\right)
$$

is called a multi-fan algebra of $\Delta$.

Remark 7.6 The constructions above show that there is a ring epimorphism from $H^{*}(\Delta) \cong \mathbb{R}[K] / \Theta$ to $\mathcal{A}^{*}(\Delta) \cong \operatorname{PD}\left(H^{*}(\Delta), \int_{\Delta}\right)$, sending $x_{i}$ to $\partial_{i}$ for each $i \in[m]$. Therefore $\mathcal{A}^{*}(\Delta)$ can be considered as a quotient of $H^{*}(\Delta)$, and all the relations in $\mathbb{R}[K] / \Theta$ are inherited by $\mathcal{A}^{*}(\Delta)$. We have

$$
\begin{aligned}
& \partial_{J} V_{\Delta}=0 \text { for } J \notin K \quad \text { (Stanley-Reisner relations), } \\
& \left(\sum_{i \in[m]} \lambda_{i, j} \partial_{i}\right) V_{\Delta}=0 \text { for } j=1, \ldots, n \quad \text { (Linear relations). }
\end{aligned}
$$

This proves points 1 and 2 of Lemma 5.1 in a more conceptual way. 


\section{Structure of Multi-Fan Algebra in Particular Cases}

\subsection{Ordinary Fans}

As was mentioned in the introduction, when $\Delta$ is a normal fan of a simple convex polytope $P$, the construction of the algebra $\mathcal{A}^{*}(\Delta)=\mathcal{D} /$ Ann $V_{\Delta}$ was introduced by Khovanskii and Pukhlikov (1992b) and studied extensively by Timorin (1999). In this case the underlying simplicial complex of $\Delta$ is a sphere and the weight function takes value +1 on all maximal simplices of $K$. Using purely combinatorial and geometrical considerations Timorin proved that $\mathcal{A}^{*}(\Delta) \cong \mathbb{R}[K] / \Theta$. This means, in particular, that dimensions $d_{i}=\operatorname{dim} \mathcal{A}^{2 i}(\Delta)$ are equal to $h_{i}$, the $h$-numbers of $K$ (see the definition below). The developed technique is applied to prove that $\mathcal{A}^{*}(\Delta)$ is a Lefschetz algebra, meaning that there exists an element $\omega \in \mathcal{A}^{2}(\Delta)$ such that

$$
\times \omega^{n-2 k}: \mathcal{A}^{2 k} \rightarrow \mathcal{A}^{2 n-2 k}
$$

is an isomorphism for each $k=0, \ldots,[n / 2]$. In particular this implies that the distribution of $h$-numbers of convex simplicial spheres is unimodal, i.e.

$$
h_{0} \leqslant h_{1} \leqslant \cdots \leqslant h_{[n / 2]}=h_{n-[n / 2]} \geqslant \cdots \geqslant h_{n-1} \geqslant h_{n} .
$$

According to Timorin's result, Lefschetz element $\omega$ may be chosen in the form $c_{1}(P)=$ $c_{1} \partial_{1}+\cdots+c_{m} \partial_{m} \in \mathcal{A}^{2}(\Delta)$ where $P$ is any convex simple polytope with the normal fan $\Delta$ and support parameters $c_{1}, \ldots, c_{m}$.

For complete non-singular fans the algebra $\mathcal{A}^{*}[\Delta] \cong \mathbb{R}[K] / \Theta$ coincides with the cohomology algebra $H^{*}\left(X_{\Delta} ; \mathbb{R}\right)$ of the corresponding toric variety. It was the original observation of Stanley (1980), that in the case when a fan $\Delta$ is polytopal, the corresponding complete toric variety $X_{\Delta}$ is projective, therefore there exists a Lefschetz element in its cohomology ring according to hard Lefschetz theorem.

After Stanley's work, several approaches were developed to prove the existence of Lefschetz elements in elementary terms, i.e. without referring to hard Lefschetz theorem. These approaches include in particular McMullen's construction of the polytope algebra (McMullen 1993), the approach based on continuous piece-wise polynomial functions (Brion 1996), and the approach based on the volume polynomial and differential operators (Timorin 1999).

We will see that ordinary fans are not the only examples of multi-fans for which the structure of $\mathcal{A}^{*}(\Delta)$ can be explicitly described. On the other hand, $\mathcal{A}^{*}(\Delta)$ is always a Poincare duality algebra, so it is natural to ask if it is Lefschetz (or at least if the dimension vector $\left(d_{0}, d_{1}, \ldots, d_{n}\right)$ is unimodal). We will show that this is not true in general, see Theorem 10.1 below. 


\subsection{Combinatorial Preliminaries}

For now we concentrate on multi-fans based on oriented pseudomanifolds as described in Example 2.9. Let $K$ be a pure simplicial complex of dimension $n-1$ on the vertex set $[m]$.

Let $f_{j}$ denote the number of $j$-dimensional simplices of $K$ for $j=-1,0, \ldots, n-1$, in particular we assume that $f_{-1}=1$ (this reflects the fact that the empty simplex formally has dimension -1 ). The $h$-numbers of $K$ are defined by the formula:

$$
\sum_{j=0}^{n} h_{j} t^{n-j}=\sum_{j=0}^{n} f_{j-1}(t-1)^{n-j}
$$

where $t$ is a formal variable. Let $\widetilde{\beta}_{j}(K)$ denote the reduced Betti number $\operatorname{dim} \widetilde{H}_{j}(K)$ of $K$. The $h^{\prime}$ - and $h^{\prime \prime}$-numbers of $K$ are defined by the formulas

$$
\begin{aligned}
& h_{j}^{\prime}=h_{j}+\left(\begin{array}{l}
n \\
j
\end{array}\right)\left(\sum_{s=1}^{j-1}(-1)^{j-s-1} \widetilde{\beta}_{s-1}(K)\right) \text { for } 0 \leqslant j \leqslant n \\
& h_{j}^{\prime \prime}=h_{j}^{\prime}-\left(\begin{array}{c}
n \\
j
\end{array}\right) \widetilde{\beta}_{j-1}(K)=h_{j}+\left(\begin{array}{c}
n \\
j
\end{array}\right)\left(\sum_{s=1}^{j}(-1)^{j-s-1} \widetilde{\beta}_{s-1}(K)\right)
\end{aligned}
$$

for $0 \leqslant j \leqslant n-1$, and $h_{n}^{\prime \prime}=h_{n}^{\prime}$. The sum over an empty set is assumed zero.

\subsection{Homology Spheres}

Definition 8.1 $K$ is called Cohen-Macaulay (over $\mathbb{k}$ ), if $\widetilde{H}_{j}\left(\operatorname{lk}_{K} I ; \mathbb{k}\right)=0$ for any $I \in K$ and $j<\operatorname{dim} \mathrm{k}_{K} I=n-1-|I|$. If, moreover, $\widetilde{H}_{n-1-|I|}\left(\mathrm{k}_{K} I ; \mathbb{k}\right) \cong \mathbb{k}$ for any $I \in K$, then $K$ is called Gorenstein* or (generalized) homology sphere.

The famous theorems of Reisner and Stanley (the reader is referred to the monograph (Stanley 1996)) tell that whenever $K$ is Cohen-Macaulay (resp. Gorenstein*), its Stanley-Reisner algebra $\mathbb{k}[K]$ is Cohen-Macaulay (resp. Gorenstein).

Given a characteristic function $\lambda:[m] \rightarrow V \cong \mathbb{R}^{n}$ we obtain a linear system of parameters $\theta_{1}, \ldots, \theta_{n} \in \mathbb{R}[K]$. It generates an ideal which we denoted by $\Theta \subset \mathbb{R}[K]$ in Sect. 4.2. In Cohen-Macaulay case every linear system of parameters is a regular sequence. This implies (Stanley 1996):

$$
\operatorname{dim}(\mathbb{R}[K] / \Theta)_{2 j}=h_{j}
$$

If $K$ is a homology sphere, then $\mathbb{R}[K]$ is Gorenstein. Thus its quotient by a linear system of parameters $\mathbb{R}[K] / \Theta$ is a Gorenstein algebra of Krull dimension zero. This implies that $\mathbb{R}[K] / \Theta$ is a Poincare duality algebra (Meyer and Smith 2005, Part 1).

Now let $\Delta$ be a complete multi-fan based on a homology sphere $K$. We have the ring epimorphism $\mathbb{R}[K] / \Theta \rightarrow \mathcal{A}^{*}(\Delta)$ (see Remark 7.6). Since both algebras 
have Poincare duality, this map is an isomorphism (see Lemma 7.4). This proves the following

Theorem 8.2 Let $\Delta$ be a complete multi-fan based on a homology sphere $K$. Then $\mathcal{A}^{*}(\Delta) \cong \mathbb{R}[K] / \Theta$. It follows that $\operatorname{dim} \mathcal{A}^{2 j}(\Delta)=h_{j}$, the h-number of $K$.

Note that Poincare duality implies the well-known Dehn-Sommerville relations for homology spheres: $h_{j}=h_{n-j}$.

We are in position to prove Lemma 6.1 which states that the volume polynomial of an elementary multi-fan $\Delta$ on the vectors $\lambda(i) \in V(i=1, \ldots, n+1)$, is equal, up to multiplicative constant, to $\left(\sum_{i=1}^{n+1} \alpha_{i} c_{i}\right)^{n}$, where $\left(\alpha_{1}, \ldots, \alpha_{n+1}\right)$ is a linear relation on $\lambda(i)$ 's.

Proof of Lemma 6.1 The underlying simplicial complex of $\Delta$ is the boundary of a simplex, which is a sphere. Therefore, by Theorem 8.2 we have $\mathcal{A}^{*}(\Delta) \cong \mathbb{R}\left[\partial \triangle_{[n+1]}\right] / \Theta$. Hence the ideal Ann $V_{\Delta} \subset \mathbb{R}\left[\partial_{1}, \ldots, \partial_{n+1}\right]$ is generated by $\prod_{i=1}^{n+1} \partial_{i}$ (Stanley-Reisner relation) and linear differential operators $\theta_{j}=\sum_{i=1}^{n+1} \lambda_{i, j} \partial_{i}$ for $j=1, \ldots, n$. Here $\left(\lambda_{i, j}\right)_{j=1}^{n}$ are the coordinates of the vector $\lambda(i)$ for each $i=1, \ldots, n+1$. Since $\sum_{i=1}^{n+1} \alpha_{i} \lambda(i)=0$ we have a linear relation $\sum_{i=1}^{n+1} \alpha_{i} \lambda_{i, j}=0$ for each $j=1, \ldots, n$. Now it is easy to check that the differential operators $\prod_{i=1}^{n+1} \partial_{i}$ and $\theta_{j}=\sum_{i=1}^{n+1} \lambda_{i, j} \partial_{i}$, $j=1, \ldots, n$ annihilate the polynomial $\left(\alpha_{1} c_{1}+\cdots+\alpha_{n+1} c_{n+1}\right)^{n}$. Thus, according to Proposition 7.2, $V_{\Delta}$ coincides with $\left(\alpha_{1} c_{1}+\cdots+\alpha_{n+1} c_{n+1}\right)^{n}$ up to constant.

\subsection{Homology Manifolds}

Definition 8.3 $K$ is called Buchsbaum (over $\mathbb{k}$ ), if $\widetilde{H}_{j}\left(\operatorname{lk}_{\widetilde{H}} I ; \mathbb{k}\right)=0$ for any $I \in K$, $I \neq \varnothing$ and $j<\operatorname{dim} \mathrm{lk}_{K} I=n-1-|I|$. If, moreover, $\widetilde{H}_{n-1-|I|}\left(\mathrm{lk}_{K} I ; \mathbb{k}\right) \cong \mathbb{k}$ for any $I \in K, I \neq \varnothing$, then $K$ is called a homology manifold. $K$ is called an orientable homology manifold if $\widetilde{H}_{n-1}(K ; \mathbb{Z}) \cong \mathbb{Z}$.

The difference from the Cohen-Macaulay case is that there are no restrictions on the topology of $K=\mathrm{lk}_{K} \varnothing$ itself. Similar to Cohen-Macaulay property, the term "Buchsbaum" indicates that the corresponding algebra $\mathbb{k}[K]$ is Buchsbaum (the result of Schenzel 1981). In Buchsbaum case linear system of parameters is no longer a regular sequence. Nevertheless, Buchsbaum complexes are extensively studied. First, Schenzel's theorem (Schenzel 1981) tells that if $K$ is a Buchsbaum complex, then

$$
\operatorname{dim}(\mathbb{k}[K] / \Theta)_{2 j}=h_{j}^{\prime}
$$

for $j=0, \ldots, n$ and the $h^{\prime}$-numbers determined by (8.2). Second, there is a theory of socles of Buchsbaum complexes introduced by Novik and Swartz (2009a) which we briefly review next.

Let $\mathcal{M}$ be a module over the graded polynomial ring $\mathbb{k}[m]:=\mathbb{k}\left[x_{1}, \ldots, x_{m}\right]$. The socle of $\mathcal{M}$ is the following subspace 


$$
\operatorname{Soc} \mathcal{M}:=\left\{a \in \mathcal{M} \mid a \cdot \mathbb{k}[m]_{+}=0\right\} \text {. }
$$

which is obviously a $\mathbb{k}[m]$-submodule of $\mathcal{M}$.

If $K$ is Buchsbaum, then there exists a submodule $I_{N S} \subset \operatorname{Soc}(\mathbb{k}[K] / \Theta)$ such that

$$
\left(I_{N S}\right)_{2 j} \cong\left(\begin{array}{c}
n \\
j
\end{array}\right) \widetilde{H}^{j-1}(K ; \mathbb{k}),
$$

where the right hand side means the direct sum of $\left(\begin{array}{l}n \\ j\end{array}\right)$ copies of $\widetilde{H}^{j-1}(K ; \mathbb{k})$. Moreover, the result of Novik and Swartz (2009b) tells that whenever $K$ is an orientable connected homology manifold, then $I_{N S}$ coincides with $\operatorname{Soc}(\mathbb{k}[K] / \Theta)$ and the quotient

$$
(\mathbb{k}[K] / \Theta) / I_{N S}^{<2 n}
$$

is a Gorenstein algebra (thus Poincare duality algebra). Here $I_{N S}^{<2 n}$ is the part of $I_{N S}$ taken in all degrees except the top one, $2 n$. The definition of $h^{\prime \prime}$-numbers (8.3) implies that

$$
\operatorname{dim}\left((\mathbb{k}[K] / \Theta) / I_{N S}^{<2 n}\right)_{2 j}=h_{j}^{\prime \prime} \text { for } 0 \leqslant j \leqslant n
$$

Now let $\Delta$ be a complete multi-fan based on an orientable connected homology manifold $K$. Recall from Definition 7.3 that $W^{*}$ denotes the subspace of $H^{*}(\Delta)=$ $\mathbb{R}[K] / \Theta$ whose graded components are

$$
\begin{aligned}
W^{2 j} & =\left\{a \in(\mathbb{R}[K] / \Theta)_{2 j} \mid \int a \cdot(\mathbb{R}[K] / \Theta)_{2 n-2 j}=0\right\} \\
& =\left\{a \in(\mathbb{R}[K] / \Theta)_{2 j} \mid \int a \cdot \mathbb{R}[m]_{2 n-2 j}=0\right\} .
\end{aligned}
$$

By definition, $\mathcal{A}^{*}(\Delta)=\operatorname{PD}(\mathbb{R}[K] / \Theta)=(\mathbb{R}[K] / \Theta) / W^{*}$. The socle $\operatorname{Soc}(\mathbb{R}[K] / \Theta)$ lies in $W^{*}$ in all degrees except the top one since it is killed by $\mathbb{R}[m]_{+}$. Therefore we have a well-defined ring epimorphism

$$
(\mathbb{R}[K] / \Theta) / I_{N S}^{<2 n} \rightarrow \mathcal{A}^{*}(\Delta) .
$$

Again, since both algebras have Poincare duality, there holds

Theorem 8.4 Let $\Delta$ be a complete multi-fan based on oriented connected homology manifold $K$. Then $\mathcal{A}^{*}(\Delta) \cong(\mathbb{R}[K] / \Theta) / I_{N S}^{<2 n}$. It follows that $\operatorname{dim} \mathcal{A}^{2 j}(\Delta)=h_{j}^{\prime \prime}$, the $h^{\prime \prime}$-number of $K$.

In this case Poincare duality implies the well-known generalized Dehn-Sommerville relations for oriented homology manifolds: $h_{j}^{\prime \prime}=h_{n-j}^{\prime \prime}$ (see Novik and Swartz 2009a and references therein). 


\subsection{General Situation}

Let $\Delta$ be an arbitrary complete multi-fan. In the largest generality we do not have a combinatorial description for the dimensions of graded components of the multi-fan algebra.

Conjecture 8.5 Let $w_{c h}$ be a simplicial cycle, $\lambda:[m] \rightarrow V$ a characteristic function, and $\Delta=\left(w_{c h}, \lambda\right)$ the corresponding complete multi-fan. The numbers $d_{j}=\operatorname{dim} \mathcal{A}^{2 j}(\Delta)$ do not depend on $\lambda$.

\section{Geometry of Multi-Polytopes and Minkowski Relations}

Here we give another proof of Theorem 8.4 which shows the geometrical nature of the elements lying in the socle of $\mathbb{R}[K] / \Theta$ when $K$ is an oriented homology manifold. It relates on explicit computations in coordinates but reveals an interesting connection with the Minkowski type relations, appearing in convex geometry. Recall the basic Minkowski theorem on convex polytopes.

\section{Theorem 1 (Minkowski)}

1. (Direct) Let $P$ be a convex full-dimensional polytope in euclidian space $\mathbb{R}^{n}$. Let $V_{1}, \ldots, V_{m}$ be the $(n-1)$-volumes of facets of $P$ and $\mathbf{n}_{1}, \ldots, \mathbf{n}_{m}$ be the outward unit normal vectors to facets. Then $\sum_{i} V_{i} \mathbf{n}_{i}=0$ (the Minkowski relation).

2. (Inverse) Let $\mathbf{n}_{1}, \ldots, \mathbf{n}_{m}$ be the vectors of unit lengths, spanning $\mathbb{R}^{n}$, and let $V_{1}, \ldots, V_{m}$ be positive numbers satisfying the Minkowski relation. Then there exists a convex polytope $P$ whose facets have outward normal vectors $\mathbf{n}_{i}$ and volumes $V_{i}$. Such polytope is unique up to parallel shifts.

Usually only part (2) is called Minkowski theorem, since part (1) is fairly simple. The direct Minkowski theorem has a straightforward generalization.

Theorem 9.1 Let $\sum_{s} a_{s} Q_{s}$ be a collection of $k$-dimensional multi-polytopes in euclidian space $\mathbb{R}^{n}$, forming a closed orientable cycle. Let $\operatorname{Vol}\left(Q_{S}\right)$ be the $k$-volume, and $v_{s} \in \Lambda^{n-k} \mathbb{R}^{n}$ be the unit normal skew form of the multi-polytope $Q_{s}$. Then there holds a relation $\sum_{s} a_{s} \operatorname{Vol}\left(Q_{s}\right) v_{s}=0$ in $\Lambda^{n-k} \mathbb{R}^{n}$.

In the next subsection we explain the precise meaning of the terms used in the statement and give the proof.

\subsection{Cycles of Multi-Polytopes}

As before, $V^{*} \cong \mathbb{R}^{n}$ denotes the ambient affine space of $n$-dimensional polytopes, coming with fixed orientation. Let $k \leqslant n$ and let $\Pi$ be an oriented $k$-dimensional affine subspace of $V^{*}$. Let $Q$ be a $k$-dimensional multi-polytope in $\Pi$. Then $Q$ will be called a $k$-dimensional multi-polytope in $V^{*}$.

First let $k>0$. Denote by $G \mathcal{M} \mathcal{P}_{k}$ the group (or a vector space over $\mathbb{R}$ ) freely generated by all $k$-dimensional multi-polytopes in $V^{*}$, where we identify the element 
$\bar{Q}$ (i.e. $Q$ with reversed orientation of the underlying subspace) and $-Q$. If $k=0$, the multi-polytope is just a point with weight. In this case let $G \mathcal{M} \mathcal{P}_{0}$ denote the group of formal sums of points whose weights sum to zero. Formally set $G \mathcal{M P} \mathcal{P}_{-1}=0$. Define the differential $d: G \mathcal{M} \mathcal{P}_{k} \rightarrow G \mathcal{M} \mathcal{P}_{k-1}$ by setting

$$
d Q:=\sum_{F_{i}: \text { facet of } Q} F_{i},
$$

and extending by linearity. Note that each facet comes with the canonical orientation: we say that the hyperplane $H_{i}$ containing $F_{i}$ is positively oriented if

(a positive basis of $H_{i}, \lambda(i)$ )

is a positive basis of $V$. Thus the expression above is well defined.

Definition 9.2 An element $A=\sum_{s} a_{s} Q_{s} \in G \mathcal{M} \mathcal{P}_{k}$ which satisfies $d A=0$ is called a cycle of $k$-dimensional multi-polytopes.

As in Sect. 5, assume that there is a fixed inner product in $V$. This allows to define the inner product on the skew forms. In particular, if $\Pi$ is an oriented affine $k$-subspace in $V^{*} \cong V$, we may define its unit normal skew form $v_{\Pi} \in \Lambda^{n-k} V$ as the unique element of $\Lambda^{n-k} \Pi^{\perp} \cong \mathbb{R}$ which corresponds to the positive orientation of $\Pi^{\perp}$ and satisfies $\left\|v_{\Pi}\right\|=1$. It is easy to see that if $\operatorname{dim} \Pi=n-1$, the form $v_{\Pi}$ is just the positive unit normal vector to $\Pi$.

Let us prove Theorem 9.1.

Proof The idea of proof is straightforward and quite similar to the proof of classical Minkowski theorem: at first we prove the case $k=n$, then reduce the general case to the case $k=n$ by projecting $\sum_{s} a_{s} \operatorname{Vol}\left(Q_{s}\right) v_{s}$ to all possible $k$-subspaces. Note that the case $n=0$ should be treated separately, but in this case the statement is trivial.

(1) Suppose $k=n$. Then all multi-polytopes $Q_{s}$ are full-dimensional. Their underlying subspaces $\Pi_{s}$ coincide with $V$ up to orientation. Without loss of generality assume that all orientations coincide with that of $V$. Normal skew forms lie in $\Lambda^{0} V \cong \mathbb{R}$ and are equal to 1 . Hence we need to prove that $\sum a_{s} \operatorname{Vol}\left(Q_{s}\right)=0$ for any cycle of $n$-multi-polytopes. Recall the wall-crossing formula (Hattori and Masuda 2003, Lemma 5.3):

Lemma 9.3 Let $P$ be a multi-polytope and $H=H_{i}$ be one of the supporting hyperplanes: $H=H_{i}$. Let $u_{\alpha}$ and $u_{\beta}$ be elements in $V^{*} \backslash \bigcup_{i=1}^{m} H_{i}$ such that the segment from $u_{\alpha}$ to $u_{\beta}$ intersects the wall $H$ transversely at $\mu$, and does not intersect any other $H_{j} \neq H$. Then

$$
\mathrm{DH}_{P}\left(u_{\alpha}\right)-\mathrm{DH}_{P}\left(u_{\beta}\right)=\sum_{i: H_{i}=H} \operatorname{sgn}\left\langle u_{\beta}-u_{\alpha}, \lambda(i)\right\rangle \mathrm{DH}_{F_{i}}(\mu)
$$

where $F_{i}$ is the facet of $P$, and $\mathrm{DH}_{F_{i}}: H_{i} \rightarrow \mathbb{R}$ is its Duistermaat-Heckman function. 
Consider a cycle of multi-polytopes $A=\sum_{s=1}^{l} a_{s} Q_{s}$. Let $\mathcal{H}$ denote the set of all supporting hyperplanes of all polytopes $Q_{s}, s=1, \ldots, l$. We have a function

$$
\mathrm{DH}_{A}: V^{*} \backslash \bigcup_{H \in \mathcal{H}} H \rightarrow \mathbb{R}, \quad \mathrm{DH}_{A}:=\sum_{s=1}^{l} a_{s} \mathrm{DH}_{Q_{s}} .
$$

Let us choose a hyperplane $H \in \mathcal{H}$ and two points $u_{\alpha}$ and $u_{\beta}$ in $V^{*} \backslash \bigcup_{H \in \mathcal{H}} H$ such that the segment from $u_{\alpha}$ to $u_{\beta}$ intersects the wall $H$ transversely at $\mu$ and does not intersect any other wall from $\mathcal{H}$. Let us sum the differences $\mathrm{DH}_{P}\left(u_{\alpha}\right)-\mathrm{DH}_{P}\left(u_{\beta}\right)$ taken with coefficients $a_{s}$ over all multi-polytopes $Q_{s}$ for which $H$ is a supporting hyperplane. Since $d A=0$, Lemma 9.3 implies that this sum is zero. Obviously, this sum equals $\mathrm{DH}_{A}\left(u_{\alpha}\right)-\mathrm{DH}_{A}\left(u_{\beta}\right)$.

This argument shows that crossing of any wall does not change the value of $\mathrm{DH}_{A}$. Therefore, $\mathrm{DH}_{A}$ is constant (where it is defined). Since $\mathrm{DH}_{A}$ has compact support, it must be constantly zero. Thus

$$
\sum a_{s} \operatorname{Vol}\left(Q_{s}\right)=\int_{V^{*}} \mathrm{DH}_{A}=0 .
$$

(2) Let us prove the theorem for general $k$. Consider a generic oriented $k$-subspace $\Pi \subset V^{*}$ and let $v \in \Lambda^{n-k} V^{*}$ be its normal skew form. Let $\Gamma: V^{*} \rightarrow \Pi$ be the orthogonal projection. Then the image of $Q_{s}$ under $\Gamma$ is a full-dimensional multipolytope in $\Pi$, which we denote by $\Gamma\left(Q_{s}\right)$. The sum $\sum_{s=1}^{l} a_{s} \Gamma\left(Q_{s}\right)$ is a cycle of $k$-dimensional multi-polytopes in $\Pi$. Therefore, step (1) implies

$$
\sum_{s=1}^{l} a_{s} \operatorname{Vol}\left(\Gamma\left(Q_{s}\right)\right)=0 .
$$

By the standard property of orthogonal projections we have

$$
\operatorname{Vol}\left(\Gamma\left(Q_{s}\right)\right)=\operatorname{Vol}\left(Q_{s}\right) \cdot\left\langle v_{s}, v\right\rangle
$$

Hence

$$
\left\langle\sum_{s=1}^{l} a_{s} \operatorname{Vol}\left(Q_{s}\right) v_{s}, v\right\rangle=0
$$

and this holds for any generic skew form $v$. Thus $\sum_{s=1}^{l} a_{s} \operatorname{Vol}\left(Q_{s}\right) v_{s}=0$ which was to be proved.

\subsection{Relations in $\mathcal{A}^{*}(\Delta)$ as Minkowski Relations}

Let $K$ be an oriented homology $(n-1)$-manifold and $\Delta$ be a multi-fan based on $K$. Suppose that every simplex $I \in K$ is oriented somehow. This defines an orientation of each subspace $H_{I}=\bigcap_{i \in I} H_{i}$ (for example, by the rule "positive orientation of 
$H_{I}^{\prime \prime} \oplus \lambda\left(i_{1}\right) \oplus \cdots \oplus \lambda\left(i_{k}\right)$ is a positive orientation of $V$ if $\left(i_{1}, \ldots, i_{k}\right)$ is a positive order of vertices of $I)$. Recall from Sect. 5 that $\lambda(I)$ denotes the skew form $\bigwedge_{i \in I} \lambda(i)$ and $\operatorname{covol}(I)=\|\lambda(I)\|$. Consider an arbitrary skew form $\mu \in \Lambda^{k} V^{*}$ and let

$$
\lambda(I)_{\mu}:=\langle\lambda(I), \mu\rangle .
$$

Let $C^{k}(K ; \mathbb{R}), 0 \leqslant k \leqslant n-1$ denote the group of cochains on $K$ and $\delta: C^{k}(K ; \mathbb{R}) \rightarrow C^{k+1}(K ; \mathbb{R})$ be the standard cochain differential. We also need to augment the cochain complex in the top degree, so we formally set $C^{n}(K ; \mathbb{R}):=\mathbb{R}$ and let $\delta: C^{n-1}(K ; \mathbb{R}) \rightarrow C^{n}(K ; \mathbb{R})$ be the evaluation of a cochain on the fundamental chain of $K$.

An element $a \in C^{k-1}(K ; \mathbb{R}), k \leqslant n$ will be called a (coaugmented) cocycle if $\delta a=0$. Then, since $K$ is an oriented manifold, the Poincare dual $\sum_{I:|I|=k} a(I) F_{I}$ of $a$ is a cycle of $(n-k)$-dimensional multi-polytopes in $V^{*}$. (Notice that in the case $k=n$ we get a formal sum of points whose weights sum is zero. If we do not require that $a$ is coaugmented, then we do not get a cycle of 0-dimensional multi-polytopes).

Proposition 9.4 For any coaugmented cocycle $a \in C^{k-1}(K ; \mathbb{R})$ and any $\mu \in \Lambda^{k} V^{*}$ there exists a relation

$$
\sum_{I:|I|=k} a(I) \lambda(I)_{\mu} \partial_{I}=0
$$

in $\mathcal{A}^{*}(\Delta) \cong(\mathbb{R}[K] / \Theta) / I_{N S}^{<2 n}$.

Proof Let us apply $\sum_{I:|I|=k} a(I) \lambda(I)_{\mu} \partial_{I}$ to the volume polynomial $V_{\Delta}$ and evaluate the result at a point $\bar{c}=\left(c_{1}, \ldots, c_{m}\right)$ :

$$
\left.\sum_{I:|I|=k} a(I) \lambda(I)_{\mu} \partial_{I} V_{\Delta}\right|_{\bar{c}}=\sum_{I:|I|=k} a(I) \lambda(I)_{\mu} \frac{\operatorname{Vol}\left(F_{I}\right)}{\operatorname{covol}(I)} .
$$

Here we used Lemma 5.1. Note that the skew form $\lambda(I) / \operatorname{covol}(I)$ is by definition a unit normal skew form to the ambient subspace of a multi-polytope $F_{I}$. Since $\sum_{I:|I|=k} a(I) F_{I}$ is a cycle of multi-polytopes, Theorem 9.1 implies

$$
\sum_{I:|I|=k} a(I) \operatorname{Vol}\left(F_{I}\right) \frac{\lambda(I)}{\operatorname{covol}(I)}=0 .
$$

Taking inner product with $\mu$ implies

$$
\sum_{I:|I|=k} a(I) \lambda(I)_{\mu} \frac{\operatorname{Vol}\left(F_{I}\right)}{\operatorname{covol}(I)}=0 .
$$

Hence the polynomial $\sum_{I:|I|=k} a(I) \lambda(I)_{\mu} \partial_{I} V_{\Delta}$ evaluates to zero at any point $\bar{c}$. Therefore it vanishes as a polynomial. Thus $\sum_{I:|I|=k} a(I) \lambda(I)_{\mu} \partial_{I} \in$ Ann $V_{\Delta}$ which proves the statement. 
We see that Minkowski theorem allows to construct linear relations in $\mathcal{A}^{*}(\Delta)$. Actually these relations exhaust all relations in $\mathcal{A}^{*}(\Delta)$. Let us state the result of Ayzenberg (2016) in terms of Minkowski relations:

Proposition 9.5 (Ayzenberg 2016) Let $K$ be an oriented homology manifold.

1. There is an isomorphism of vector spaces

$$
(\mathbb{R}[K] / \Theta)_{2 k} \cong\left\langle x_{I}|I \in K,| I \mid=k\right\rangle /\left\langle\sum_{I:|I|=k} a(I) \lambda(I)_{\mu} x_{I}\right\rangle
$$

where a runs over all exact $(k-1)$-cochains on $K$ and $\mu$ runs over $\mu \in \Lambda^{k} V^{*}$.

2. There is an isomorphism of vector spaces

$$
\left((\mathbb{R}[K] / \Theta) / I_{N S}^{<2 n}\right)_{2 k} \cong\left\langle x_{I}|I \in K,| I \mid=k\right\rangle /\left\langle\sum_{I:|I|=k} a(I) \lambda(I)_{\mu} x_{I}\right\rangle
$$

where a runs over all coaugmented closed $(k-1)$-cochains on $K$ and $\mu$ runs over $\mu \in \Lambda^{k} V^{*}$.

Recall that $\left(I_{N S}\right)_{2 k} \cong\left(\begin{array}{l}n \\ k\end{array}\right) H^{k-1}(K ; \mathbb{R})$. From Proposition 9.5 it can be seen that the difference between the vector spaces $\mathbb{R}[K] / \Theta$ and $(\mathbb{R}[K] / \Theta) / I_{N S}^{<2 n}$ arises from the difference between closed cochains on $K$ and exact cochains. This explains how the cohomology $H^{k-1}(K)$ appears in the description of $I_{N S}$. The multiple $\left(\begin{array}{l}n \\ k\end{array}\right)$ comes from the choice of the skew form $\mu \in \Lambda^{k} V$ on which the Minkowski relation is projected.

Problem 9.6 Let $\Delta$ be a general complete simplicial multi-fan. Is it true that $\mathcal{A}^{*}(\Delta)$ is isomorphic, as a vector space, to the quotient of $\left\langle x_{I} \mid I \in K\right\rangle$ by linear relations arising from Minkowski relations? What are these Minkowski relations?

\subsection{Inverse Minkowski Theorem}

It is tempting to formulate and prove the inverse Minkowski theorem for multipolytopes. First, we need to modify the statement. The original formulation tells that there exists a convex polytope with the given normal vectors and the volumes of facets, but it tells nothing about the combinatorics of the polytope. We may ask a more specific question, namely

Question 9.7 For a given complete simplicial multi-fan $\Delta$ with the rays generated by unit vectors $\mathbf{n}_{1}, \ldots, \mathbf{n}_{m}$, and a given $m$-tuple of real numbers $V_{1}, \ldots, V_{m}$ satisfying $\sum V_{i} \mathbf{n}_{i}=0$, does there exist a multi-polytope based on $\Delta$ whose facets have $(n-1)$ volumes $V_{1}, \ldots, V_{m}$ ? If yes, is it unique?

A simple example shows that the answer, even for the question of existence, may be negative. 
Example 9.8 Let $\Delta$ be the normal fan of a 3-dimensional cube. $\Delta$ is an ordinary fan supported by a simplicial complex $K$, which is the boundary of an octahedron. Let $\{1,2,3,-1,-2,-3\}$ be the set of vertices of $K$ and $\lambda( \pm 1)=( \pm 1,0,0)$, $\lambda( \pm 2)=(0, \pm 1,0), \lambda( \pm 3)=(0,0, \pm 1)$ be the generators of the corresponding rays of $\Delta$ (see Example 6.6). The multi-polytopes based on $\Delta$ are the bricks with sides parallel to coordinate axes. Minkowski relations can be written as $\operatorname{Vol}\left(F_{i}\right)=\operatorname{Vol}\left(F_{-i}\right)$ for $i=1,2,3$. Let us take the numbers $V_{ \pm 1}=0, V_{ \pm 2}=V_{ \pm 3}=1$. These numbers satisfy Minkowski relations, but there are no bricks whose facets have volumes $V_{ \pm 1}, V_{ \pm 2}, V_{ \pm 3}$. Indeed, $V_{ \pm 1}=0$ implies that one of the sides of a brick has length 0 but this would imply that either $V_{ \pm 2}=0$ or $V_{ \pm 3}=0$.

Nevertheless, the answer to Question 9.7 is completely controlled by the multi-fan algebra. Recall that $\mathcal{A}^{*}(\Delta)$ may be interpreted as the algebra of differential operators $\mathcal{D}$ up to $\operatorname{Ann}\left(V_{\Delta}\right)$. Therefore, for every $a \in \mathcal{A}^{2 j}(\Delta)$, there is a well-defined homogeneous polynomial $a V_{\Delta}$ of degree $n-j$. In particular, each element $a \in \mathcal{A}^{2 n-2}(\Delta)$ determines a linear homogeneous polynomial $a V_{\Delta}=V_{1} c_{1}+\cdots+V_{m} c_{m} \in \mathbb{R}\left[c_{1}, \ldots, c_{m}\right]_{1}$. This linear polynomial is annihilated by $\theta_{j}=\sum_{i \in[m]} \lambda_{i, j} \partial_{j} \in$ Ann $V_{\Delta}, j=1, \ldots, n$, see Lemma 5.1 or Remark 7.6. This means

$$
\sum_{i \in[m]} V_{i} \lambda(i)=0,
$$

which can be considered as a Minkowski relation. Let Mink denote the vector space of all $m$-tuples $\left(V_{1}, \ldots, V_{m}\right)$ satisfying (9.1). Thus we obtain a natural monomorphism $\eta: \mathcal{A}^{2 n-2}(\Delta) \rightarrow$ Mink, $a \mapsto\left(V_{1}, \ldots, V_{m}\right)$, where $a V_{\Delta}=V_{1} c_{1}+\cdots+V_{m} c_{m}$.

Theorem 9.9 Let $\Delta$ be a complete simplicial multi-fan with characteristic function $\lambda$ and assume that $|\lambda(i)|=1$ for each $i \in[m]$. Let $\bar{V}=\left(V_{1}, \ldots, V_{m}\right) \in$ Mink. Let $P \in \operatorname{Poly}(\Delta)$ be a multi-polytope and $\partial_{P}=c_{1} \partial_{1}+\cdots+c_{m} \partial_{m} \in \mathcal{A}^{2}(\Delta)$ be its first Chern class. Then the polytope $P$ has facet volumes $V_{1}, \ldots, V_{m}$ if and only if $\eta\left(\partial_{P}^{n-1}\right)=(n-1) ! \bar{V}$.

Proof Assume that $\eta\left(\partial_{P}^{n-1}\right)=(n-1) ! \bar{V}$. Then $\partial_{P}^{n-1} V_{\Delta}=(n-1) !\left(V_{1} c_{1}+\cdots+\right.$ $\left.V_{m} c_{m}\right)$. Hence $\partial_{i} \partial_{P}^{n-1} V_{\Delta}=(n-1) ! V_{i}$ for $i \in[m]$. On the other hand, Corollary 5.7 implies

$\partial_{i} \partial_{P}^{n-1} V_{\Delta}=(n-1) ! \operatorname{Vol}\left(F_{i}\right) / \operatorname{covol}(i)=(n-1) ! \operatorname{Vol}\left(F_{i}\right) /|\lambda(i)|=(n-1) ! \operatorname{Vol}\left(F_{i}\right)$.

Thus $V_{i}=\operatorname{Vol}\left(F_{i}\right)$. The opposite direction is proved similarly.

Note that $\operatorname{dim}$ Mink $=m-n$.

Corollary 9.10 Existence in Question 9.7 holds for a given multi-fan $\Delta$ and all $m$ tuples $\left(V_{1}, \ldots, V_{m}\right) \in$ Mink if and only if the following two conditions hold:

1. $\operatorname{dim} \mathcal{A}^{2}(\Delta)=\operatorname{dim} \mathcal{A}^{2 n-2}(\Delta)=m-n$;

2. the power map $\mathcal{A}^{2}(\Delta) \rightarrow \mathcal{A}^{2 n-2}(\Delta)$, $\partial \mapsto \partial^{n-1}$ is surjective.

Uniqueness holds if the power map is bijective. 
Remark 9.11 Note that even the condition $\operatorname{dim} \mathcal{A}^{2}(\Delta)=m-n$ may fail to hold. As an example, consider a multi-fan having a ghost vertex, say 1 . As in general, we have $n$ relations $\theta_{1}, \ldots, \theta_{n}$, lying in the kernel of the linear map $\left\langle\partial_{1}, \ldots, \partial_{m}\right\rangle \rightarrow \mathcal{A}^{2}(\Delta)$. But the element $\partial_{1}$, corresponding to the ghost vertex, also vanishes in $\mathcal{A}^{2}(\Delta)$. Thus $\operatorname{dim} \mathcal{A}^{2}(\Delta)<m-n$.

There exist more nontrivial examples. For example, if the underlying simplicial complex $K$ is disconnected, with connected components $K_{1}, \ldots, K_{r}$ on disjoint vertex sets $\left[m_{1}\right], \ldots,\left[m_{r}\right], r>1$, then each connected component contributes at most $m_{1}-n$ in the total dimension of $\mathcal{A}^{2}(\Delta)$ (see the operation of connected sum of Poincare duality algebras introduced in Sect. 11.1). Thus in the disconnected case $\operatorname{dim} \mathcal{A}^{2}(\Delta) \leqslant m-r n$, where $m=m_{1}+\cdots+m_{r}$. Nevertheless, the inverse Minkowski theorem can be refined in an obvious way: we should consider Minkowski relations on each connected component.

Remark 9.12 The power map $\mathcal{A}^{2}(\Delta) \rightarrow \mathcal{A}^{2 n-2}(\Delta)$ is a polynomial map of degree $n-1$ between real vector spaces of equal dimensions. It is a complicated object which may be interesting on its own. One of the consequences from Corollary 9.10 is that the existence in the inverse Minkowski theorem holds for a multi-fan $\Delta$ whenever $\operatorname{dim} \mathcal{A}^{2}(\Delta)=m-n$ and $n$ is even.

\section{Recognizing Volume Polynomials and Multi-Fan Algebras}

A natural question is: which homogeneous polynomials are the volume polynomials, and which Poincare duality algebras appear as $\mathcal{A}^{*}(\Delta)$ ? The answer to the second question seems quite unexpected.

Theorem 10.1 For every Poincare duality algebra $\mathcal{A}^{*}$ generated in degree 2 there exists a complete simplicial multi-fan $\Delta$ such that $\mathcal{A}^{*} \cong \mathcal{A}^{*}(\Delta)$.

Recall that the symmetric array of nonnegative integers $\left(d_{0}, d_{1}, \ldots, d_{n}\right), d_{j}=$ $d_{n-j}$, is called unimodal, if

$$
d_{0} \leqslant d_{1} \leqslant \cdots \leqslant d_{\lfloor n / 2\rfloor} .
$$

Corollary 10.2 There exist multi-fans $\Delta$, for which the array

$$
\left(\operatorname{dim} \mathcal{A}^{0}(\Delta), \operatorname{dim} \mathcal{A}^{2}(\Delta), \ldots, \operatorname{dim} \mathcal{A}^{2 n}(\Delta)\right)
$$

is not unimodal.

Proof An example of Poincare duality algebra generated in degree 2, for which dimensions of graded components are not unimodal was given by Stanley (1978). Theorem 10.1 implies that there exists a multi-fan, which produces this algebra.

The construction of the volume polynomial is linear with respect to weights. Let MultiFans $_{\lambda}$ denote the vector space of all multi-fans with the given characteristic function $\lambda:[m] \rightarrow V$. Then we obtain a linear map 


$$
\Omega_{\lambda}: \text { MultiFans }_{\lambda} \rightarrow \mathbb{R}\left[c_{1}, \ldots, c_{m}\right]_{n},
$$

which maps $\Delta$ to its volume polynomial $V_{\Delta}$.

Before giving the proof of Theorem 10.1 we characterize volume polynomials of general position, in the sense explained below. For this goal we study the properties of the map $\Omega_{\lambda}$.

\subsection{Characterization of Volume Polynomials in General Position}

There is a necessary condition on $V_{\Delta}$. If $\lambda$ is a characteristic function and $\theta_{j}=$ $\sum_{i \in[m]} \lambda_{i, j} \partial_{i} \in\left\langle\partial_{1}, \ldots, \partial_{m}\right\rangle, j=1, \ldots, n$ are the corresponding linear forms, then $\theta_{j} V_{\Delta}=0$, see Remark 7.6. Thus the subspace

$$
\mathrm{Ann}^{2} V_{\Delta}=\left\{D \in\left\langle\partial_{1}, \ldots, \partial_{m}\right\rangle \mid D V_{\Delta}=0\right\}
$$

has dimension at least $n$. It happens that in most situations this is also a sufficient condition for a polynomial to be a volume polynomial.

At first let us consider the situation of general position to demonstrate the argument. Assume that all characteristic vectors $\lambda(1), \ldots, \lambda(m) \in \mathbb{R}^{n}$ are in general position, which means that every $n$ of them are linearly independent. Given a fixed characteristic function $\lambda:[m] \rightarrow V$ in general position, we may pick up any simplicial cycle $w_{c h} \in Z\left(\triangle_{[m]}^{(n-1)} ; \mathbb{R}\right)$, consider a complete multi-fan $\Delta=(w, \lambda)$ and take its volume polynomial. This defines a map which we previously denoted by $\Omega_{\lambda}$ :

$$
\Omega_{\lambda}: Z\left(\triangle_{[m]}^{(n-1)} ; \mathbb{R}\right) \rightarrow \mathbb{R}\left[c_{1}, \ldots, c_{m}\right]_{n},
$$

from the $(n-1)$-simplicial cycles on $m$ vertices to homogeneous polynomials of degree $n$. This map is linear and injective by Corollary 5.5. As before, let $\theta_{j}, j=1, \ldots, n$ be the linear differential operators associated with $\lambda$ (i.e. a basis of the image of the $\left.\operatorname{map} \lambda^{\top}: V^{*} \rightarrow\left(\mathbb{R}^{m}\right)^{*}\right)$. Let

$$
\operatorname{Ann}^{n} \Theta=\left\{\Psi \in \mathbb{R}\left[c_{1}, \ldots, c_{m}\right]_{n} \mid \theta_{j} \Psi=0 \text { for each } j=1, \ldots, n\right\}
$$

be the vector subspace of polynomials annihilated by differential operators $\Theta=$ $\left(\theta_{1}, \ldots, \theta_{n}\right)$. As we have seen, if $\Delta$ has a characteristic function $\lambda$, then $V_{\Delta} \in \operatorname{Ann}^{n} \Theta$. Thus the image of $\Omega_{\lambda}$ lies in $\mathrm{Ann}^{n} \Theta$.

Lemma 10.3 If $\lambda$ is in general position, then $\Omega_{\lambda}: Z_{n-1}\left(\triangle_{[m]}^{(n-1)} ; \mathbb{R}\right) \rightarrow \operatorname{Ann}^{n} \Theta$ is an isomorphism.

Proof Let us compute the dimensions of the domain and the target. There are no $n$-simplices in $\triangle_{[m]}^{(n-1)}$, thus $Z_{n-1}\left(\triangle_{[m]}^{(n-1)}\right)=H_{n-1}\left(\triangle_{[m]}^{(n-1)}\right)$. All Betti numbers of $\triangle_{[m]}^{(n-1)}$ between the top and the bottom vanish, thus via Euler characteristic we get

$$
\operatorname{dim} H_{n-1}\left(\triangle_{[m]}^{(n-1)}\right)=\left(\begin{array}{c}
m \\
n
\end{array}\right)-\left(\begin{array}{c}
m \\
n-1
\end{array}\right)+\left(\begin{array}{c}
m \\
n-2
\end{array}\right)-\cdots+(-1)^{n}\left(\begin{array}{c}
m \\
0
\end{array}\right) .
$$


Now let us compute $\operatorname{dim} \mathrm{Ann}^{n} \Theta$. Take a linear change of variables $c_{1}, \ldots, c_{m} \rightsquigarrow$ $c_{1}^{\prime}, \ldots, c_{m}^{\prime}$ such that $\theta_{j}$ becomes the partial derivative $\frac{\partial}{\partial c_{j}^{\prime}}$ for $j=1, \ldots, n$. Thus, after the change of variables, $\mathrm{Ann}^{n} \Theta$ becomes the set $\left\{\Psi \in \mathbb{R}\left[c_{1}^{\prime}, \ldots, c_{m}^{\prime}\right]_{n} \mid \frac{\partial}{\partial c_{j}^{\prime}} \Psi=\right.$ $0, j=1, \ldots, n\}$ which is the same as $\mathbb{R}\left[c_{n+1}^{\prime}, \ldots, c_{m}^{\prime}\right]_{n}$. Thus $\operatorname{dim} \operatorname{Ann}^{n} \Theta=$ $\left(\begin{array}{c}m-n+n-1 \\ n\end{array}\right)=\left(\begin{array}{c}m-1 \\ n\end{array}\right)$. This number coincides with (10.2).

Let $G_{m, n}$ denote the Grassmann manifold of all (unoriented) $n$-planes in $\left(\mathbb{R}^{m}\right)^{*}$. We can introduce the standard Plücker coordinates on $G_{m, n}$. If

$$
\left\{\theta_{j}=\sum_{i=1}^{m} \lambda_{i, j} x_{i}\right\}_{j=1, \ldots, n}
$$

is a basis in $L \in G_{m, n}$, then the Plücker coordinates of $L$ are all maximal minors of the $m \times n$ matrix $\left(\lambda_{i, j}\right)$.

Any $n$-plane $L \in G_{m, n}$ determines an $m$-tuple of vectors in $V \cong \mathbb{R}^{n}$ as follows: the basis $(10.3)$ determines the tuple $\left\{\lambda(i)=\left(\lambda_{i, 1}, \ldots, \lambda_{i, n}\right)\right\}_{i \in[m]}$. The base change in $L$ induces the natural action of $\operatorname{GL}(n, \mathbb{R})$ on the $m$-tuples. By abuse of terminology we call $\lambda:[m] \rightarrow V$ the characteristic function corresponding to $L \in G_{m, n}$ although this characteristic function is determined only up to automorphism of $V$.

Proposition 10.4 Let $\Psi \in \mathbb{R}\left[c_{1}, \ldots, c_{m}\right]_{n}$ be a homogeneous polynomial. Suppose that the vector subspace $\mathrm{Ann}^{2} \Psi=\left\{D \in\left\langle\partial_{1}, \ldots, \partial_{m}\right\rangle \mid D \Psi=0\right\}$ contains an $n$-dimensional subspace $L \in G_{m, n}$ with all Plücker coordinates non-zero. Then $\Psi$ is a volume polynomial of some multi-fan.

Proof Let us pick a basis $\left\{\theta_{j}=\sum_{i} \lambda_{i, j} x_{i}\right\}_{j=1, \ldots, n}$ in $L$ arbitrarily. Non-vanishing of all Plücker coordinates means that the corresponding characteristic function $\lambda$ is in general position. By assumption, $\Psi \in \mathrm{Ann}^{n} \Theta$. Thus $\Psi$ is a volume polynomial of some multi-fan based on $\lambda$ according to Lemma 10.3.

\subsection{Proof of Theorem 10.1}

Let $\mathcal{A}^{*}$ be an arbitrary Poincare duality algebra over $\mathbb{R}$ generated by $\mathcal{A}^{2}$. Let $2 n$ be the formal dimension of $\mathcal{A}$ and $p=\operatorname{dim} \mathcal{A}^{2}$. Take any $p+n$ elements $x_{1}, \ldots, x_{p+n} \in \mathcal{A}^{2}$ in general position (i.e. every $p$ of them are linearly independent). There are $n$ linear relations on $x_{1}, \ldots, x_{p+n}$ in $\mathcal{A}^{2}$ of the form $\sum_{i} \lambda_{i, j} x_{i}=0, j=1, \ldots, n$. Since $x_{i}$ are in general position, every maximal minor of the $(p+n) \times n$-matrix $\left|\lambda_{i, j}\right|$ is non-zero.

As in the proof of Proposition 7.2, consider the polynomial

$$
\Psi_{\mathcal{A}}\left(c_{1}, \ldots, c_{m}\right)=\frac{1}{n !} \int\left(c_{1} x_{1}+\cdots+c_{m} x_{m}\right)^{n},
$$


where $\int: \mathcal{A}^{2 n} \stackrel{\cong}{\mathbb{R}}$ is any isomorphism. The linear differential operator $\theta_{j}=$ $\sum_{i} \lambda_{i, j} \partial_{i}$ annihilates $\Psi_{\mathcal{A}}$ for $j=1, \ldots, n$. Indeed:

$$
\begin{aligned}
& \left(\sum_{i=1}^{m} \lambda_{i, j} \frac{\partial}{\partial c_{i}}\right) \frac{1}{n !} \int\left(c_{1} x_{1}+\cdots+c_{m} x_{m}\right)^{n} \\
& =\frac{1}{n !} \cdot n \int\left(\sum_{i=1}^{m} \lambda_{i, j} x_{i}\right)\left(c_{1} x_{1}+\cdots+c_{m} x_{m}\right)^{n-1}=0 .
\end{aligned}
$$

Since $\theta_{j}$ are in general position, Proposition 10.4 implies that $\Psi_{\mathcal{A}}=V_{\Delta}$ for some multi-fan $\Delta$. Therefore the corresponding Poincare duality algebras $\mathcal{A}^{*}$ and $\mathcal{A}^{*}(\Delta)$ are isomorphic by Proposition 7.2.

\subsection{Non-General Position}

Now we want to study which polynomials are volume polynomials without the assumption of general position.

Let $I \subset[m]$ and let $\alpha_{I}: \mathbb{R}^{I} \rightarrow \mathbb{R}^{m}$ be the inclusion of the coordinate subspace. Then $\alpha_{I}^{*}:\left(\mathbb{R}^{m}\right)^{*}=\left\langle\partial_{1}, \ldots, \partial_{m}\right\rangle \rightarrow\left(\mathbb{R}^{I}\right)^{*}$ is a projection map. For a linear subspace $\Pi \subset\left(\mathbb{R}^{m}\right)^{*}$ of dimension at least $n$ consider the following collection of subsets of $[m]$ :

$$
\operatorname{dep}(\Pi):=\left\{I \subset[m]|| I \mid \leqslant n \text { and }\left.\alpha_{I}^{*}\right|_{\Pi}: \Pi \rightarrow\left(\mathbb{R}^{I}\right)^{*} \text { is not surjective }\right\} .
$$

Lemma 10.5 Let $\Pi \subset\left(\mathbb{R}^{m}\right)^{*}$ and $\operatorname{dim} \Pi \geqslant n$. Then there exists a subspace $L \subset \Pi$ such that $\operatorname{dim} L=n$ and $\operatorname{dep}(L)=\operatorname{dep}(\Pi)$.

Proof When $\operatorname{dim} \Pi=n$ the statement is trivial so we assume $\operatorname{dim} \Pi>n$. The proof follows from the general position argument. If $\varphi: \Pi \rightarrow U$ is an epimorphism, and $\operatorname{dim} \Pi>n \geqslant \operatorname{dim} U$, then the set of all $n$-planes in $\Pi$ which map surjectively to $U$ is a complement to a subvariety of positive codimension inside the set of all $n$-subspaces of $\Pi$. This argument applied to all maps $\left.\alpha_{I}^{*}\right|_{\Pi}: \Pi \rightarrow\left(\mathbb{R}^{I}\right)^{*}$ proves that any generic $n$-plane $L$ in $\Pi$ satisfies $\operatorname{dep}(L)=\operatorname{dep}(\Pi)$.

Let $\Psi$ be a homogeneous polynomial of degree $n$ and $\operatorname{Ann}^{2} \Psi \subset\left\langle\partial_{1}, \ldots, \partial_{m}\right\rangle=$ $\left(\mathbb{R}^{m}\right)^{*}$ be its annihilator subspace.

Theorem 10.6 A homogeneous polynomial $\Psi \in \mathbb{R}\left[c_{1}, \ldots, c_{m}\right]_{n}$ is a volume polynomial of some complete simplicial multi-fan if and only if the following conditions hold:

1. $\operatorname{dim} \mathrm{Ann}^{2} \Psi \geqslant n$,

2. $\partial_{I} \Psi=0$ whenever $I \in \operatorname{dep}\left(\operatorname{Ann}^{2} \Psi\right)$.

Proof The necessity of these conditions is already proved. Indeed, the first condition follows from the fact that $\mathrm{Ann}^{2} V_{\Delta}$ contains the image of $\lambda^{\top}: V^{*} \rightarrow\left(\mathbb{R}^{m}\right)^{*}=$ 
$\left\langle\partial_{1}, \ldots, \partial_{m}\right\rangle$ which has dimension $n$, see Remark 7.6. If $I \in \operatorname{dep}\left(\operatorname{Ann}^{2} V_{\Delta}\right)$, then *-condition (see Sect. 2.2) implies $I \notin K$, and therefore $\partial_{I} V_{\Delta}=0$ by Lemma 5.1.

Let us prove sufficiency. By Lemma 10.5 we may choose an $n$-dimensional plane $L \subset \operatorname{Ann}^{2} \Psi$ such that $\operatorname{dep}(L)=\operatorname{dep}\left(\operatorname{Ann}^{2} \Psi\right)$. Therefore, by assumption, $I \in \operatorname{dep}(L)$ implies $\partial_{I} \Psi=0$. Let $\lambda:[m] \rightarrow V$ be the characteristic function corresponding to $L \in G_{m, n}$. The condition $I \in \operatorname{dep}(L)$ is equivalent to the condition that vectors $\{\lambda(i)\}_{i \in I}$ are linearly dependent.

Consider a simplicial complex $M a t r_{\lambda}$ determined by the condition: $\left\{i_{1}, \ldots, i_{k}\right\} \in$ Matr $_{\lambda}$ if and only if $\lambda\left(i_{1}\right), \ldots, \lambda\left(i_{k}\right)$ are linearly independent. Thus $\operatorname{Matr}_{\lambda}=$ $2^{[m]} \backslash \operatorname{dep}(L)$. In a sense, the complex $\operatorname{Matr}_{\lambda}$ can be considered as a maximal simplicial complex on $[m]$ for which $\lambda$ is a characteristic function (this construction is similar to the universal complexes introduced in Davis and Januszkiewicz (1991)).

We have $I \notin M a t r_{\lambda}$ if and only if $\Theta \rightarrow\left\langle\partial_{i}\right\rangle_{i \in I}$ is not surjective. It is easily seen that multi-fans having characteristic function $\lambda$ are encoded by the simplicial $(n-1)$ cycles on $M a t r_{\lambda}$. As before, we have a map $\Omega_{\lambda}: Z_{n-1}\left(M a t r_{\lambda} ; \mathbb{R}\right) \rightarrow \mathbb{R}\left[c_{1}, \ldots, c_{m}\right]_{n}$ which associates a volume polynomial $V_{\Delta}$ with a multi-fan $\Delta=\left(w_{c h}, \lambda\right)$ for $w_{c h} \in$ $Z_{n-1}\left(\operatorname{Matr}_{\lambda} ; \mathbb{R}\right)$. Let

$$
\operatorname{Ann}^{n}\left(L,\left\{\partial_{I}\right\}_{I \in \operatorname{dep}(L)}\right)
$$

denote the subspace of all homogeneous polynomials of degree $n$ which are annihilated by linear differential operators from $L$ and by the products $\partial_{I}, I \in \operatorname{dep}(L)(\Leftrightarrow I \notin$ $\left.M a t r_{\lambda}\right)$. We already proved that the image of $\Omega_{\lambda}$ lies in $\operatorname{Ann}^{n}\left(L,\left\{\partial_{I}\right\}_{I \in \operatorname{dep}(L)}\right)$. We need to prove that the map

$$
\Omega_{\lambda}: Z_{n-1}\left(\operatorname{Matr}_{\lambda} ; \mathbb{R}\right) \rightarrow \operatorname{Ann}^{n}\left(L,\left\{\partial_{I}\right\}_{I \in \operatorname{dep}(L)}\right)
$$

is surjective. Since $\Omega_{\lambda}$ is injective, it is enough to show that dimensions of the two spaces are equal.

First of all notice that $\operatorname{Matr}_{\lambda}$ is by construction the underlying simplicial complex of a linear matroid. Hence $M a t r_{\lambda}$ is a Cohen-Macaulay complex of dimension $n-1$ (see e.g. Stanley 1977). The number $\operatorname{dim} Z_{n-1}\left(\operatorname{Matr}_{\lambda} ; \mathbb{R}\right)=\operatorname{dim} \widetilde{H}_{n-1}\left(\operatorname{Matr}_{\lambda} ; \mathbb{R}\right)$ coincides with the top h-number $h_{n}\left(M_{a t} r_{\lambda}\right)$ of the Cohen-Macaulay complex Matr ${ }_{\lambda}$.

Consider the Stanley-Reisner ring $\mathbb{R}\left[\right.$ Matr $\left._{\lambda}\right]=\mathbb{R}\left[\partial_{1}, \ldots, \partial_{m}\right] /\left(\partial_{I} \mid I \notin M a t r_{\lambda}\right)$, and its quotient by a linear system of parameters $L \subset\left\langle\partial_{1}, \ldots, \partial_{m}\right\rangle$ :

$$
\mathbb{R}\left[M a t r_{\lambda}\right] /(L)=\mathbb{R}\left[\partial_{1}, \ldots, \partial_{m}\right] /\left(L,\left\{\partial_{I}\right\}_{I \in \operatorname{dep}(L)}\right)
$$

Claim 10.7 $\operatorname{dim}\left(\mathbb{R}\left[\operatorname{Matr}_{\lambda}\right] /(L)\right)_{2 n}=\operatorname{dim} \operatorname{Ann}^{n}\left(L,\left\{\partial_{I}\right\}_{I \in \operatorname{dep}(L)}\right)$.

This follows from basic linear algebra. There is a non-degenerate pairing

$$
\mathbb{R}\left[\partial_{1}, \ldots, \partial_{m}\right]_{2 n} \otimes \mathbb{R}\left[c_{1}, \ldots, c_{m}\right]_{n} \rightarrow \mathbb{R}
$$

For any subspace $U \subset \mathbb{R}\left[\partial_{1}, \ldots, \partial_{m}\right]_{2 n}$ we have $\operatorname{dim} \mathbb{R}\left[\partial_{1}, \ldots, \partial_{m}\right]_{2 n} / U=$ $\operatorname{dim} U^{\perp}$. Taking the degree $2 n$ part of the ideal $\left(L,\left\{\partial_{I}\right\}_{I \in \operatorname{dep}(L)}\right)$ as $U$ proves the claim. 
Now, since $M a t r_{\lambda}$ is Cohen-Macaulay, the socle of $\mathbb{R}\left[M a t r_{\lambda}\right] /(L)$ coincides with $\left(\mathbb{R}\left[\operatorname{Matr}_{\lambda}\right] /(L)\right)_{2 n}$. On the other hand, this dimension equals $h_{n}\left(M_{a t r_{\lambda}}\right)$, the top $h$ number, as follows from the basic theory of Stanley-Reisner rings (Stanley 1996). We have

$$
\operatorname{dim} \operatorname{Ann}^{n}\left(L,\left\{\partial_{I}\right\}_{I \in \operatorname{dep}(L)}\right)=\operatorname{dim} \operatorname{Soc} \mathbb{R}\left[\operatorname{Matr}_{\lambda}\right] /(L)_{2 n}=\operatorname{dim} \widetilde{H}_{n-1}\left(\text { Matr }_{\lambda} ; \mathbb{R}\right)
$$

which finishes the proof of the theorem.

Remark 10.8 Lemma 10.3 describing the general position is a particular case of Theorem 10.6. In the case of general position the matroid complex $M a t r_{\lambda}$ is just the $(n-1)$-skeleton of a simplex on $m$ vertices.

\subsection{Global Structure of the Set of Multi-Fans}

Let $G_{m}^{n}$ denote the Grassmannian of all codimension $n$ planes in $\mathbb{R}^{m} \cong \mathbb{R}\left[c_{1}, \ldots, c_{m}\right]_{1}$. Obviously, $G_{m, n}$ can be identified with $G_{m}^{n}$ by assigning $L^{\perp} \subset \mathbb{R}^{m}$ to $L \subset\left(\mathbb{R}^{m}\right)^{*}$. We have already seen, that characteristic function $\lambda: \mathbb{R}^{m} \rightarrow V$ determines the element $L \in G_{m, n}$ defined as the image of $\lambda^{\top}: V^{*} \rightarrow\left(\mathbb{R}^{m}\right)^{*}$. The corresponding element of $G_{m}^{n}$ is the subspace $Y=L^{\perp}=\operatorname{Ker} \lambda \subset \mathbb{R}^{m}$.

Let $S^{k} Y$ denote the $k$-th symmetric power of $Y \in G_{m}^{n}$, so we have $S^{k} Y \subset S^{k} \mathbb{R}^{m}=$ $\mathbb{R}\left[c_{1}, \ldots, c_{m}\right]_{k}$. We have

$$
S^{k} Y \subset \operatorname{Ann}^{k} Y^{\perp}=\left\{\Psi \in \mathbb{R}\left[c_{1}, \ldots, c_{m}\right]_{k} \mid D \Psi=0 \text { for any } D \in Y^{\perp}\right\},
$$

and both spaces have dimension $\left(\begin{array}{c}m-n+k-1 \\ k\end{array}\right)$. This implies that the vector bundle

$$
\left\{(Y, \Psi) \in G_{m}^{n} \times \mathbb{R}\left[c_{1}, \ldots, c_{m}\right]_{k} \mid \Psi \in \operatorname{Ann}^{k} Y^{\perp}\right\} \rightarrow G_{m}^{n}
$$

is $S^{k} \gamma$, the $k$-th symmetric power of the canonical bundle $\gamma$ over $G_{m}^{n}$. We denote its total space by $E\left(S^{k} \gamma\right)$.

Consider the set of all characteristic functions in $V$ up to linear automorphism of $V$ :

$$
\text { CharFunc }:=\left\{\lambda: \mathbb{R}^{m} \rightarrow V \mid \operatorname{Im} \lambda=V\right\} / \operatorname{GL}(V) .
$$

Let MultiFans denote the set of all complete simplicial multi-fans on the set $[\mathrm{m}]$ (considered up to automorphisms of $V$ again). We have a map MultiFans $\rightarrow$ CharFunc which maps a multi-fan to its characteristic function. The fiber of this map over $\lambda$ is the vector space MultiFans $\lambda \cong Z_{n-1}\left(\right.$ Matr $\left._{\lambda} ; \mathbb{R}\right)$ introduced earlier.

We have a commutative square

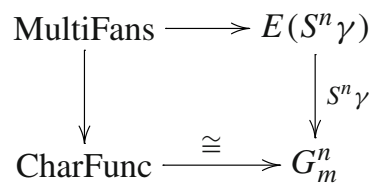


The lower map associates a codimension $n$ subspace $Y=\operatorname{Ker} \lambda$ to a characteristic function $\lambda$. The upper map associates a volume polynomial to a multi-fan. The upper map is linear on each fiber. The subset of characteristic functions in general position maps isomorphically to the subset of $G_{m}^{n}$ with non-zero Plücker coordinates; the fiber over a generic point maps isomorphically according to Lemma 10.3. Exceptional fibers map injectively and their images are described by Theorem 10.6.

\section{Surgery of Multi-Fans and Algebras}

In this section we study the behavior of the dimensions $d_{j}=\operatorname{dim} \mathcal{A}^{2 j}(\Delta)$ under connected sums and flips of multi-fans.

\subsection{Connected Sums}

Recall that $\mathcal{A}^{*}(\Psi)=\mathbb{R}\left[\partial_{1}, \ldots, \partial_{m}\right] / \operatorname{Ann}(\Psi)$ denotes the Poincare duality algebra associated with the homogeneous polynomial $\Psi$. For a graded algebra (or a graded vector space) $A^{*}=\bigoplus_{j} A^{2 j}$ let $\operatorname{Hilb}\left(A^{*} ; t\right)=\sum_{j}\left(\operatorname{dim} A^{j}\right) t^{j}$ denote its Hilbert function. Sometimes it will be convenient to use the notation $\overline{d m}\left(A^{*}\right):=\left(d_{0}, d_{1}, \ldots, d_{n}\right)$, where $d_{j}=\operatorname{dim} A^{2 j}$, and $\overline{d m}(\Delta):=\overline{d m}\left(\mathcal{A}^{*}(\Delta)\right)$.

Let $\mathcal{A}_{1} \# \mathcal{A}_{2}$ denote a connected sum of two Poincare duality algebras of the same formal dimension $2 n$. By definition, $\mathcal{A}_{1} \# \mathcal{A}_{2}=\mathcal{A}_{1} \oplus \mathcal{A}_{2} / \sim$ where $\sim$ identifies $\mathcal{A}_{1}^{0}$ with $\mathcal{A}_{2}^{0}$ and $\mathcal{A}_{1}^{2 n}$ with $\mathcal{A}_{2}^{2 n}$. Actually, there is an ambiguity in the choice of the latter identification, so in fact there exists a 1-dimensional family of connected sums of the given two algebras. We prefer to ignore this ambiguity in the following (the statements hold for any representative in the family).

We have $\overline{d m}\left(A_{1} \# A_{2}\right)=\overline{d m}\left(A_{1}\right)+\overline{d m}\left(A_{2}\right)-(1,0, \ldots, 0,1)$.

Lemma 11.1 Let $\Psi_{1} \in \mathbb{R}\left[c_{1}, \ldots, c_{m}\right]_{n}, \Psi_{2} \in \mathbb{R}\left[c_{1}^{\prime}, \ldots, c_{m^{\prime}}^{\prime}\right]_{n}$ be the polynomials in distinct variables. Then $\mathcal{A}^{*}\left(\Psi_{1}+\Psi_{2}\right) \cong \mathcal{A}^{*}\left(\Psi_{1}\right) \# \mathcal{A}^{*}\left(\Psi_{2}\right)$.

Proof The mixed differential operators $\partial_{i} \partial_{i^{\prime}}^{\prime}$ vanish on $\Psi_{1}+\Psi_{2}$, while $\partial_{I}\left(\Psi_{1}+\Psi_{2}\right)$ equals $\partial_{I}\left(\Psi_{1}\right)\left(\operatorname{resp} . \partial_{I}\left(\Psi_{2}\right)\right)$ if $I \subset[m]$ (resp. $\left.I \subset\left[m^{\prime}\right]\right)$.

Let $\Delta_{1}, \Delta_{2}$ be two multi-fans, whose vertex sets are $[m]=\{1, \ldots, n, n+1, \ldots, m\}$ and $[\widetilde{m}]=\{1, \ldots, n, \widetilde{n+1}, \ldots, \widetilde{m}\}$ respectively, and let $I$ denote the set of common vertices: $I=\{1, \ldots, n\}$. Assume that the weight of $I$ is non-zero in both multifans and assume that characteristic functions of $\Delta_{1}$ and $\Delta_{2}$ coincide on $I$. Then we may consider $\Delta_{1}$ and $\Delta_{2}$ as multi-fans with vertices $[m] \cup_{I}[\tilde{m}]=\{1, \ldots, n, n+$ $1, \ldots, m, \widetilde{n+1}, \ldots, \widetilde{m}\}$ and a common characteristic function. In this case we call the cone-wise sum $\Delta_{1}+\Delta_{2}$ a connected sum and denote it by $\Delta_{1} \#_{I} \Delta_{2}$ or simply $\Delta_{1} \# \Delta_{2}$.

Remark 11.2 It would be natural to assume that $w_{1}(I)=-w_{2}(I)$, so that the cone spanned by $I$ contracts in the connected sum. This is consistent with the geometrical understanding how "the connected sum" should look like. However, we do not need this assumption in the following proposition. 
Proposition 11.3 For a connected sum $\Delta_{1} \# \Delta_{2}$ there holds

$$
\mathcal{A}^{*}\left(\Delta_{1} \# \Delta_{2}\right) \cong \mathcal{A}^{*}\left(\Delta_{1}\right) \# \mathcal{A}^{*}\left(\Delta_{2}\right)
$$

so that $\overline{d m}\left(\Delta_{1} \# \Delta_{2}\right)=\overline{d m}\left(\Delta_{1}\right)+\overline{d m}\left(\Delta_{2}\right)-(1,0, \ldots, 0,1)$.

Proof We need a technical lemma

Lemma 11.4 Let $\Delta$ be a multi-fan and $I \subset[m]$ be a vertex set such that the corresponding characteristic vectors $\{\lambda(i)\}_{i \in I}$ are linearly independent. Let $V_{\Delta}$ be the volume polynomial and $V_{\Delta \backslash I} \in \mathbb{R}\left[c_{i} \mid i \in[m] \backslash I\right]_{n}$ be the homogeneous polynomial obtained by specializing $c_{i}=0$ in $V_{\Delta}$ for each $i \in I$. Then $\mathcal{A}^{*}\left(V_{\Delta \backslash I}\right) \cong$ $\mathcal{A}^{*}\left(V_{\Delta}\right)\left(=\mathcal{A}^{*}(\Delta)\right)$ as Poincare duality algebras.

Proof Using linear relations $\theta_{j}=\sum_{i=1}^{m} \lambda_{i, j} \partial_{i}=0$ in $\mathcal{A}^{2}(\Delta)$ we can exclude the variables $\partial_{i}$ for $i \in I$. This proves that the set $\left\{\partial_{i}\right\}_{i \in[m] \backslash I}$ spans $\mathcal{A}^{2}(\Delta)$. Therefore the polynomial

$$
V_{\Delta \backslash I}=\frac{1}{n !} \int_{\Delta}\left(\sum_{i \in[m] \backslash I} c_{i} x_{i}\right)^{n}
$$

determines the same Poincare duality algebra as $V_{\Delta}$.

By the lemma we have $\mathcal{A}^{*}\left(\Delta_{1}\right) \cong \mathcal{A}^{*}\left(V_{\Delta_{1} \backslash I}\right)$ and $\mathcal{A}^{*}\left(\Delta_{2}\right) \cong \mathcal{A}^{*}\left(V_{\Delta_{2} \backslash I}\right)$. Polynomials $V_{\Delta_{1} \backslash I}$ and $V_{\Delta_{2} \backslash I}$ have distinct variables, thus

$$
\mathcal{A}^{*}\left(V_{\Delta_{1} \backslash I}+V_{\Delta_{2} \backslash I}\right) \cong \mathcal{A}^{*}\left(V_{\Delta_{1} \backslash I}\right) \# \mathcal{A}^{*}\left(V_{\Delta_{2} \backslash I}\right)
$$

according to Lemma 11.1. It remains to note that $V_{\Delta_{1} \backslash I}+V_{\Delta_{2} \backslash I}$ is the result of specializing $c_{i}=0$ for $i \in I$ in the polynomial $V_{\Delta_{1}}+V_{\Delta_{2}}$. Finally, we have

$$
\begin{aligned}
\mathcal{A}^{*}\left(\Delta_{1} \# \Delta_{2}\right) & =\mathcal{A}^{*}\left(V_{\Delta_{1} \# \Delta_{2}}\right) \cong \mathcal{A}^{*}\left(V_{\Delta_{1} \# \Delta_{2} \backslash I}\right) \\
& \cong \mathcal{A}^{*}\left(V_{\Delta_{1} \backslash I}+V_{\Delta_{2} \backslash I}\right) \cong \mathcal{A}^{*}\left(\Delta_{1}\right) \# \mathcal{A}^{*}\left(\Delta_{2}\right) .
\end{aligned}
$$

Remark 11.5 In the proof we actually did not need that $I$ forms a cone of $\Delta_{1}$ or $\Delta$. We only need that the values of characteristic function in vertices of $I$ are linearly independent. Therefore, the connected sum may be defined in this more general setting.

\subsection{Flips}

In this section we assume that $\Delta$ is based on an oriented pseudomanifold $K$. Our goal is to define a flip in a multi-fan. Consider separately two situations.

(1) Flips changing the number of vertices. Let us take a maximal simplex $I \in K$, $|I|=n$. Let Flip $_{I}^{1}(K)$ be a simplicial complex whose maximal simplices are the same 
as in $K$ except that we substitute $I$ by Cone $\partial I$. This operation adds the new vertex $i$, the apex of the cone. If $\lambda:[m] \rightarrow \mathbb{R}^{n}$ is a characteristic function on $K$, we extend it to the set $[m] \sqcup\{i\}$ by adding new value $\lambda(i)$ such that the result is a characteristic function on $\operatorname{Flip}_{I}^{1}(K)$. This defines an operation on multi-fans which we call the flip of type $(1, n)$.

The inverse operation will be denoted Flip ${ }_{i}^{n}$. It is applicable to $\Delta$ if $\mathrm{lk}_{K} i$ is isomorphic to the boundary of a simplex and $\lambda\left(\operatorname{Vert}\left(\operatorname{lk}_{K} i\right)\right)$ is a linearly independent set. The inverse operation will be called the flip of type $(n, 1)$.

(2) Flips preserving the set of vertices. Let $S$ be a subset of $\operatorname{Vert}(K)$ of cardinality $n+1$ such that the induced subcomplex $K_{S}$ on the set $S$ is isomorphic to $\partial \Delta^{p-1} * \Delta^{q-1}$ with $p+q=n+1, p, q \geqslant 2$. Let $\operatorname{Flip}_{S}^{p}(K)$ be the simplicial complex whose maximal simplices are the same as in $K$ away from $S$, and $\partial \Delta^{p-1} * \Delta^{q-1}$ is replaced by $\Delta^{p-1} * \partial \Delta^{q-1}$. If the set of vectors $\lambda(S)$ is in general position, then this operation is defined on multi-fans. We call it the flip of type $(p, q)$. It is easily seen that flips of types $(p, q)$ and $(q, p)$ are inverse to each other.

Of course $(1, n)$ - and $(n, 1)$-flips can be viewed as particular cases of this construction if we allow ghost vertices and formally set $\partial \Delta^{0}$ to be such a ghost vertex.

The following proposition tells that dimension vectors of multi-fan algebras change under the flips similar to $h$-vectors of simplicial complexes.

Theorem 11.6 Let $\Delta^{\prime}$ be a multi-fan obtained from $\Delta$ by a $(p, q)$-flip, $p, q \geqslant 1$. Then

$$
\overline{d m}\left(\Delta^{\prime}\right)-\overline{d m}(\Delta)=(\underbrace{1, \ldots, 1}_{q}, 0, \ldots, 0)-(\underbrace{1, \ldots, 1}_{p}, 0, \ldots, 0) .
$$

Proof For $(1, n)$ - and $(n, 1)$-flips this follows from Proposition 11.3 and Lemma 6.1, since $(1, n)$-flip is just the connected sum with an elementary multi-fan, and $(n, 1)$-flip is its inverse.

Now we consider the remaining cases. $(p, q)$-flips with $p \neq 1$ and $q \neq 1$ do not change the vertex set. Let $[m]$ denote the vertex set of $K$ and $K^{\prime}$, and $S \subset[m]$ denote the set of vertices at which the flip is performed. We have $|S|=n+1$ and $\left.K\right|_{S} \cong \partial \Delta^{p-1} * \Delta^{q-1}$ and $\left.K^{\prime}\right|_{S} \cong \Delta^{p-1} * \partial \Delta^{q-1}$. Let $[p]$ be the set of vertices of $\Delta^{p-1}$. Let $\mathcal{I}_{[m] \backslash S}$ denote the ideal in $\mathbb{R}\left[\partial_{1}, \ldots, \partial_{m}\right]$ generated by $\partial_{i},(i \in[m] \backslash S)$.

Claim $11.7 \mathcal{I}_{[m] \backslash S} \cap$ Ann $V_{\Delta}=\mathcal{I}_{[m] \backslash S} \cap$ Ann $V_{\Delta^{\prime}}$.

Proof In the group of multi-fans with a given characteristic function we have a relation $\Delta^{\prime}=\Delta+T$, where $T$ is an elementary multi-fan based on the vertex set $S$. Informally, to perform a flip on a multi-fan is the same as "to add a boundary of a simplex", which cancels the cones from $\partial \Delta^{p-1} * \Delta^{q-1}$ and adds the cones from $\Delta^{p-1} * \partial \Delta^{q-1}$. Therefore $V_{\Delta^{\prime}}=V_{\Delta}+V_{T}$, where $V_{T}$ is the polynomial which essentially depends only on the variables $c_{i}, i \in S$. If $D \in$ Ann $V_{\Delta} \cap \mathcal{I}_{[m] \backslash S}$ then $D$ annihilates both $V_{\Delta}$ and $V_{T}$. Thus it annihilates $V_{\Delta^{\prime}}=V_{\Delta}+V_{T}$ and the claim follows. 
We have a diagram of inclusions of graded ideals in $\mathbb{R}\left[\partial_{1}, \ldots, \partial_{m}\right]$ :

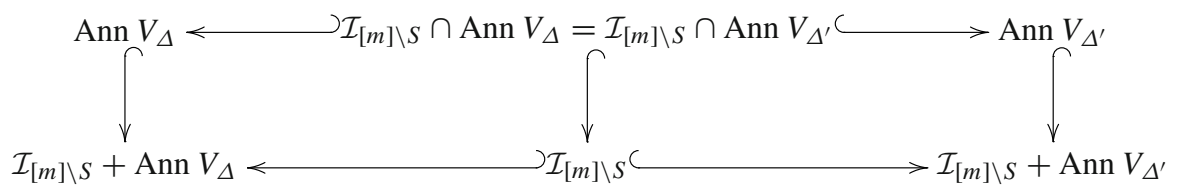

It follows that the quotients of the vertical inclusions are isomorphic as graded vector spaces. Therefore

$$
\begin{aligned}
\overline{d m}\left(\Delta^{\prime}\right)-\overline{d m}(\Delta)= & \overline{d m}\left(\mathbb{R}[m] / \text { Ann } V_{\Delta^{\prime}}\right)-\overline{d m}\left(\mathbb{R}[m] / \text { Ann } V_{\Delta}\right) \\
= & \overline{d m}\left(\mathbb{R}[m] /\left(\mathcal{I}_{[m] \backslash S}+\text { Ann } V_{\Delta^{\prime}}\right)\right) \\
& -\overline{d m}\left(\mathbb{R}[m] /\left(\mathcal{I}_{[m] \backslash S}+\text { Ann } V_{\Delta}\right)\right) .
\end{aligned}
$$

Since $\mathcal{I}_{[m] \backslash S}$ is the ideal generated by $\partial_{i},(i \notin S)$, the ring $\mathbb{R}[m] /\left(\mathcal{I}_{[m] \backslash S}+\right.$ Ann $\left.V_{\Delta}\right)$ coincides with some quotient ring $B$ of the polynomials in variables $\partial_{i},(i \in S)$, that is $B=\mathbb{R}[S] /$ Rels. The linear relations $\theta_{j}=\sum_{i \in[m]} \lambda_{i, j} \partial_{i}$ in Ann $V_{\Delta}$ induce the relations $\sum_{i \in S} \lambda_{i, j} \partial_{i}$ in Rels. Since the values of $\lambda$ on $S$ are in general position, these induced relations are linearly independent. We have $n$ linear relations on $n+1$ variables, thus all variables are expressed through a single variable $t$, and we have $B \cong \mathbb{R}[t] / \mathcal{J}$. Since we are in a graded situation, and $B$ is a finite dimensional algebra, $\mathcal{J}$ is a principal ideal generated by $t^{\tilde{p}}$ for some $\tilde{p} \geqslant 0$. Hence

$$
\overline{d m}\left(\mathbb{R}[m] /\left(\mathcal{I}_{[m] \backslash S}+\operatorname{Ann} V_{\Delta}\right)\right)=\overline{d m} B=(\underbrace{1, \ldots, 1}_{\tilde{p}}, 0, \ldots, 0) .
$$

Now notice that there is a Stanley-Reisner relation $\prod_{i \in[p]} \partial_{i}=0$ corresponding to non-simplex $[p]$ in $K$ (recall that $[p]$ is the set of vertices of $\partial \Delta^{p-1}$ inside $\partial \Delta^{p-1} *$ $\left.\Delta^{q-1} \subset K\right)$. Therefore we have a relation $t^{p}=0$ in $B$. This implies $\tilde{p} \leqslant p$.

Applying the same arguments to $\Delta^{\prime}$, we get

$$
\overline{d m}\left(\mathbb{R}[m] /\left(\mathcal{I}_{[m] \backslash S}+\operatorname{Ann} V_{\Delta^{\prime}}\right)\right)=(\underbrace{1, \ldots, 1}_{\tilde{q}}, 0, \ldots, 0),
$$

where $\tilde{q} \leqslant q$. Hence we have

$$
\overline{d m}\left(\Delta^{\prime}\right)-\overline{d m}(\Delta)=(\underbrace{1, \ldots, 1}_{\tilde{q}}, 0, \ldots, 0)-(\underbrace{1, \ldots, 1}_{\tilde{p}}, 0, \ldots, 0) .
$$

Note that the vector on the left hand side is symmetric. Hence the vector on the right hand side is symmetric. If at least one inequality $\tilde{p} \leqslant p$ or $\tilde{q} \leqslant q$ is strict, the vector at the right is not symmetric. Thus $\tilde{p}=p, \tilde{q}=q$, and the statement follows. 


\section{Cohomology of Torus Manifolds}

\subsection{Multi-Fans of Torus Manifolds}

Recall that a torus manifold $X$ is an oriented closed manifold of dimension $2 n$ with an effective action of $n$-dimensional compact torus $T$ having at least one fixed point, and prescribed orientations of characteristic submanifolds. Any torus manifold determines a non-singular multi-fan in the Lie algebra $L(T) \cong \mathbb{R}^{n}$ of the torus as follows (see details in Hattori and Masuda 2003).

Let $X_{i}, i \in[m]$ be the characteristic submanifolds. Let $M$ be a connected component of a non-empty intersection $X_{i_{1}} \cap \cdots \cap X_{i_{k}}$ for some $k>0$ and $\left\{i_{1}, \ldots, i_{k}\right\} \subset[m]$, and assume that $M$ has at least one fixed point. Such submanifold will be called $a$ face submanifold. We also assume that the manifold $X$ itself is a face submanifold corresponding to $k=0$. It easily follows from the transversality of characteristic submanifolds that $M$ has codimension $2 k$. Let $\Sigma_{X}$ be a poset of all face submanifolds of $X$ ordered by reversed inclusion. The basic representation theory of a torus implies that $\Sigma_{X}$ is a pure simplicial poset of dimension $n-1$ on the vertex set [ $m$ ]. The maximal simplices of $\Sigma_{X}$ correspond to the fixed points of $X$.

Given orientations of $X$ and $X_{i}, i \in[m]$, each fixed point obtains a sign. This determines a sign function $\sigma_{X}: \Sigma_{X}^{\langle n\rangle} \rightarrow\{-1,+1\}$.

Finally, let $T_{i}$ denote a circle subgroup fixing $X_{i}$, for $i \in[m]$. The orientation of $X_{i}$ determines the orientation of the 2-dimensional normal bundle of $X_{i}$, which in turn determines an orientation of $T_{i}$. Therefore we have a well-defined primitive element

$$
\lambda_{X}(i) \in \operatorname{Hom}\left(S^{1}, T^{n}\right) \cong \mathbb{Z}^{n} \subset \mathbb{R}^{n} \cong L\left(T^{n}\right) .
$$

This gives a characteristic function $\lambda_{X}:[m] \rightarrow \mathbb{R}^{n}$. These constructions determine a multi-fan $\Delta_{X}:=\left(\Sigma_{X}, \sigma_{X}, \lambda_{X}\right)$ associated with a torus manifold $X$. This multi-fan is non-singular and complete (Hattori and Masuda 2003).

As described in Sect. 2.2, we may turn the data "simplicial poset + sign function" into the data "simplicial complex + weight function". Let $K_{X}$ and $w_{X}$ denote the simplicial complex and the weight function corresponding to $\Delta_{X}$.

In the following we assume that each non-empty intersection of characteristic submanifolds is connected and contains at least one fixed point. This assumption implies, in particular, that $\Sigma_{X}$ is a simplicial complex, and therefore $K_{X}=\Sigma_{X}$ and the weight function $w_{X}$ coincides with $\sigma_{X}$.

\subsection{Face Subalgebra in Cohomology}

Let $X$ be a torus manifold and $\Delta_{X}$ be the corresponding multi-fan. Let $\mathcal{F}^{*}(X) \subset$ $H^{*}(X ; \mathbb{R})$ be the vector subspace spanned by the cohomology classes Poincare dual to face submanifolds. Since the intersection of two face submanifolds is either a face submanifold or empty, $\mathcal{F}^{*}(X)$ is a subalgebra. This subalgebra is multiplicatively generated by the classes of characteristic submanifolds. 
The simplices of $K_{X}=\Sigma_{X}$ correspond to face submanifolds, and there exists a ring homomorphism $\mathbb{R}\left[K_{X}\right] \rightarrow H_{T}^{*}(X)$ defined as follows: the element $x_{I}=\prod_{i \in I} x_{i} \in$ $\mathbb{R}\left[K_{X}\right]_{2|I|}$ corresponding to the simplex $I \in K_{X}$ maps to the equivariant cohomology class dual to the face submanifold $X_{I}=\bigcap_{i \in I} X_{i}$. There is a natural homomorphism $H_{T}^{*}(X) \rightarrow H^{*}(X)$ induced by the inclusion of a fiber in the Borel fibration $X \hookrightarrow$ $X \times_{T} E T \stackrel{\pi}{\rightarrow} B T$. We have a commutative square of algebra homomorphisms

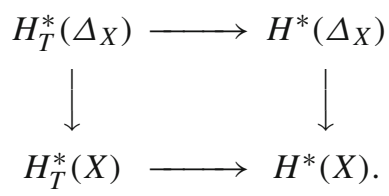

Recall from the end of Sect. 4.2 that $H_{T}^{*}\left(\Delta_{X}\right)$ denotes the Stanley-Reisner ring of the underlying simplicial complex $K_{X}, H^{*}\left(\Delta_{X}\right)$ is its quotient by the linear system of parameters, so the upper horizontal arrow in the diagram (12.1) is the natural quotient homomorphism. By definition, $\mathcal{F}^{*}(X)$ is the image of the right vertical map. Hence we have an epimorphism of algebras $H^{*}\left(\Delta_{X}\right) \rightarrow \mathcal{F}^{*}(X)$.

Theorem 12.1 There exists a well-defined epimorphism of algebras $\mathcal{F}^{*}(X) \rightarrow$ $\mathcal{A}^{*}\left(\Delta_{X}\right)$.

Proof The epimorphism $H^{*}\left(\Delta_{X}\right) \rightarrow \mathcal{F}^{*}(X)$ is compatible with the integration maps $\int_{\Delta_{X}}: H^{2 n}\left(\Delta_{X}\right) \rightarrow \mathbb{R}$ (the multi-fan integration) and $\int_{X}: \mathcal{F}^{2 n}(X) \rightarrow \mathbb{R}$ (integration over the manifold $X$ ), see Hattori and Masuda (2003). Lemma 7.4 implies that the induced map $\operatorname{PD}\left(H^{*}\left(\Delta_{X}\right), \int_{\Delta_{X}}\right) \rightarrow \operatorname{PD}\left(\mathcal{F}^{*}(X), \int_{X}\right)$ is an isomorphism. Thus we have a natural epimorphism $\mathcal{F}^{*}(X) \rightarrow \operatorname{PD}\left(\mathcal{F}^{*}(X), \int_{X}\right) \cong \operatorname{PD}\left(H^{*}\left(\Delta_{X}\right), \int_{\Delta_{X}}\right)=$ $\mathcal{A}^{*}\left(\Delta_{X}\right)$.

Therefore the part of the cohomology ring generated by characteristic submanifolds is clamped between two algebras defined combinatorially:

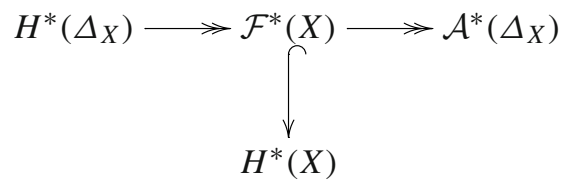

Corollary 12.2 Betti numbers of a torus manifold $X$ are bounded below by the dimensions of graded components of $\mathcal{A}^{*}\left(\Delta_{X}\right)$.

Remark 12.3 For complete smooth toric varieties and for quasitoric manifolds all arrows in the diagram above are isomorphisms as follows from Danilov-Jurkiewicz and Davis-Januszkiewicz (Davis and Januszkiewicz 1991) theorems respectively. This fact was later generalized to simplicial posets by Masuda and Panov (2006). If $K_{X}$ is a sphere, both horizontal maps are isomorphisms, so the face part of cohomology is completely determined by a multi-fan, while the vertical map may be non-trivial. As an example in which such phenomena occur, take an equivariant connected sum of a 
(quasi)toric manifold with a manifold on which the torus acts freely and whose orbit space is not a homology sphere. Finally, there exist many examples of torus manifolds for which all arrows in (12.2) are nontrivial (Ayzenberg 2016).

Recall that $H^{*}\left(\Delta_{X}\right)$ is linearly generated by the square-free monomials $x_{I}$ corresponding to simplices $I \in K$. There may exist certain linear relations on these elements coming from Minkowski relations: $\sum_{I:|I|=k} a(I) \lambda(I)_{\mu} x_{I}$, where $\mu \in \Lambda^{k} V$ and $a$ is a function on $(k-1)$-simplices of $K$.

Conjecture 12.4 As a vector space, $\mathcal{F}^{2 k}(X)$ is generated by the elements $\left\{x_{I}\right\}_{I \in K}$ subject to the Minkowski relations $\sum_{I:|I|=k} a(I) \lambda(I)_{\mu} x_{I}=0$, where $\mu$ runs over $\Lambda^{k} V$ and a runs over all functions such that the element

$$
\sum_{I:|I|=k} a(I)\left[X_{I} / T\right]
$$

bounds in $C_{n-k}(X / T ; \mathbb{R})$.

This question is closely related to Problem 9.6. It can be seen that whenever $\sum_{I:|I|=k} a(I)\left[X_{I} / T\right]$ bounds in $C_{n-k}(X / T ; \mathbb{R})$, the element $\sum_{I:|I|=k} a(I) F_{I}$ is a cycle of multi-polytopes, therefore $\sum_{I:|I|=k} a(I) \lambda(I)_{\mu} x_{I}$ vanishes in $\mathcal{A}^{*}\left(\Delta_{X}\right)$. However, there may be cycles of multi-polytopes such that the corresponding elements $\sum_{I:|I|=k} a(I)\left[X_{I} / T\right]$ do not bound in the orbit space. This observation represents the fact that the right arrow in (12.2) can be nontrivial.

If $X$ is an oriented manifold with locally standard torus action, having trivial free part and acyclic proper faces of the orbit space, the conjecture is proved in Ayzenberg (2016). Informally, this situation corresponds to the case when the underlying simplicial complex of $\Delta_{X}$ is an oriented homology manifold.

\section{References}

Ayzenberg A.: Homology cycles in manifolds with locally standard torus actions. Homol. Homotopy Appl. 18(1), 1-23 (2016). doi:10.4310/HHA.2016.v18.n1.a1

Berchtold, F.: Lifting of morphisms to quotient presentations. Manuscr. Math. 110, 33-44 (2003)

Brion, M.: Piecewise Polynomial Functions, Convex Polytopes and Enumerative Geometry, vol. 36, pp. 25-44. Banach Center Publications, Institute of Mathematics, Polish Academy of Sciences, Warszawa (1996)

Buchstaber, V., Panov, T.: Toric Topology. Mathematical Surveys and Monographs, vol. 204. AMS, Providence (2015)

Davis, M., Januszkiewicz, T.: Convex polytopes, Coxeter orbifolds and torus actions. Duke Math. J. 62(2), 417-451 (1991)

Fulton, W.: Introduction to Toric Varieties. Princeton University Press, Princeton (1993)

Hattori, A., Masuda, M.: Theory of multi-fans. Osaka J. Math. 40, 1-68 (2003)

Khovanskii, A.G., Pukhlikov, A.V.: Finitely additive measures of virtual polyhedra. Algebra i Analiz 4(2), 161-185 (1992a) [English transl., St. Petersburg Math. J. 4, 337-356 (1992)]

Khovanskii, A.G., Pukhlikov, A.V.: The Riemann-Roch theorem for integrals and sums of quasipolynomials on virtual polytopes. Algebra i Analiz 4(4), 188-216 (1992b) [English transl., St. Petersburg Math. J. 4(4), 789-812 (1993)]

Lawrence, J.: Polytope volume computation. Math. Comput. 57, 259-271 (1991)

Masuda, M., Panov, T.: On the cohomology of torus manifolds. Osaka J. Math. 43, 711-746 (2006) 
McMullen, P.: On simple polytopes. Invent. Math. 113, 419-444 (1993)

Meyer, D.M., Smith, L.: Poincaré Duality Algebras, Macaulay's Dual Systems, and Steenrod Operations. Cambridge Tracts in Mathematics 167, Cambridge University Press (2005)

Novik, I., Swartz, Ed.: Socles of Buchsbaum modules, complexes and posets. Adv. Math. 222, 2059-2084 (2009a)

Novik, I., Swartz, E.: Gorenstein rings through face rings of manifolds. Compos. Math. 145, 993-1000 (2009b)

Schenzel, P.: On the number of faces of simplicial complexes and the purity of Frobenius. Math. Zeitschrift 178, 125-142 (1981)

Stanley, R.: Cohen-Macaulay complexes. In: Aigner, M. (ed.) Higher Combinatorics, pp. 51-62. NATO Advanced Study Institute Series/Reidel, Dordrecht/Boston (1977)

Stanley, R.: Hilbert functions of graded algebras. Adv. Math. 28, 57-83 (1978)

Stanley, R.: The number of faces of a simplicial convex polytope. Adv. Math. 35, 236-238 (1980)

Stanley, R.: Combinatorics and Commutative Algebra. Progress in Mathematics, vol. 41. Birkhäuser Boston Inc., Boston (1996)

Timorin, V.A.: An analogue of the Hodge-Riemann relations for simple convex polytopes. Russ. Math. Surv. 54(2), 381-426 (1999) 